\title{
One-pot synthesis of 2,5-dihydrosiloles and their silole-annulated analogs starting from alkynylsilanes with a terminal alkynyl group
}

\author{
Oleg L. Tok, ${ }^{\mathrm{a} *}$ Jonathan Bould, ${ }^{\mathrm{a}}$ Michal Dušek ${ }^{\mathrm{b}}$, Josef Cvačka $^{\mathrm{c}}$

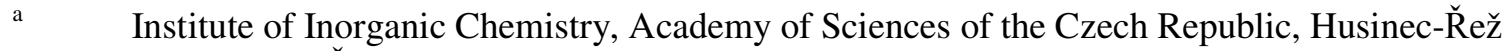 \\ 1001, 25068 Řež, Czech Republic \\ E-mail: tok@iic.cas.cz \\ b Institute of Physics, Academy of Sciences of the Czech Republic, Na Slovance 1999/2, 18221, \\ Praha 8, Czech Republic \\ c Institute of Organic Chemistry and Biochemistry, Academy of Sciences of the Czech Republic, \\ Flemingovo nam. 2, 16610 Prague 6, Czech Republic
}

\begin{abstract}
In contrast to the reaction of vinyl(alkynyl)silanes with 9-BBN-H, leading to the quantitative formation of 5-R-4-(9-BBN)-2,3-dihydro-1H-siloles, the treatment of bis(alkynyl)silanes bearing one terminal alkynyl group with 2 equivalents of 9-BBN-H, followed by methanolysis, afforded 5-R-4-(9BBN)-2,5-dihydro-1H-siloles with the yields $85-90 \%$ (according the NMR monitoring). The reaction proceeds via a double 1,2-hydroboration of the terminal triple bond with the formation of the geminal diborane followed by ring closure via intramolecular 1,1-carboboration of the remaining alkynyl fragment. Depending on the nature of the substituent $\mathrm{R}$ in position 5, the allylic BBN-group locates in position $3(\mathrm{R}=\mathrm{Ph})$ or position $5\left(\mathrm{R}=\mathrm{SiHMe}_{2}, \mathrm{SiMe}_{3}\right)$ to give 2,3- or 2,5-dihydrosiloles, respectively. The protodeborylation of the allylic BBN-group with $\mathrm{MeOH}$ of both 3,4-(9-BBN $)_{2}$-2,3-dihydro- and $4,5(9-\mathrm{BBN})_{2}-2,5$-dihydrosiloles results in the exclusive formation of 4-BBN-2,5-dihydrosiloles. In all cases, the formation of $10-12 \%$ of $2-\mathrm{R}-2,4(9-\mathrm{BBN})_{2}-2,3$-dihydrosilole minor isomers has been observed, which occur from vicinal diboranes formed as side products by a second hydroboration of the terminal triple bond. Similarly, the treatment of the tri- and tetraalkynes containing a terminal triple bond with 2 equivalents of 9-BBN-H, followed by treatment with methanol, resulted in the high yield formation of 1,2,6,6a-tetrahydro-1,6-disilapentalenes and 2,6,7,7a-tetrahydro-1,6,7-trisila-1H-cyclopenta[a]pentalenes, respectively.
\end{abstract}

\section{Supporting Information}

$\underline{\text { Table of Contents }}$

1. ${ }^{1} \mathrm{H},{ }^{13} \mathrm{C}$ and ${ }^{29} \mathrm{Si}$ NMR spectra $\quad$ S2

2. X-ray crystallographic analysis details $\quad$ S37

3. DFT calculations details $\quad$ S39

4. Kinetic study of the equilibrium between $\mathbf{5 d}$ and 7d $\quad$ S49

5. FTIR spectra of $\mathbf{5 d} / \mathbf{7 d}$ and $\mathbf{1 6 d} \quad$ S53

6. References $\quad$ S54 


\section{1. ${ }^{1} \mathrm{H},{ }^{13} \mathrm{C}$ and ${ }^{29} \mathrm{Si}$ NMR spectra.}

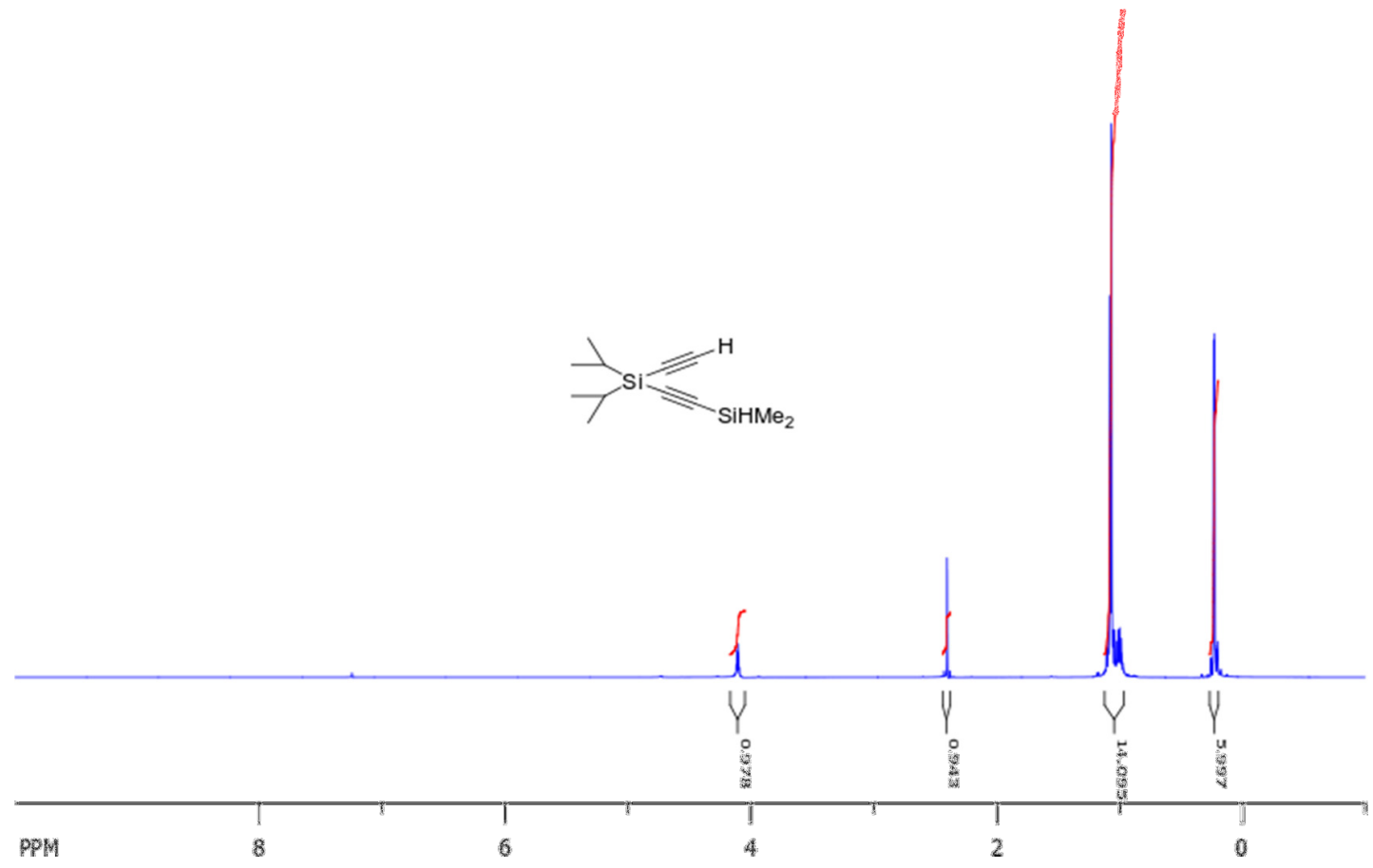

Figure S1. ${ }^{1} \mathrm{H}$ NMR spectrum $\left(600 \mathrm{MHz}, \mathrm{CDCl}_{3}\right)$ of $\mathbf{1 d}$.
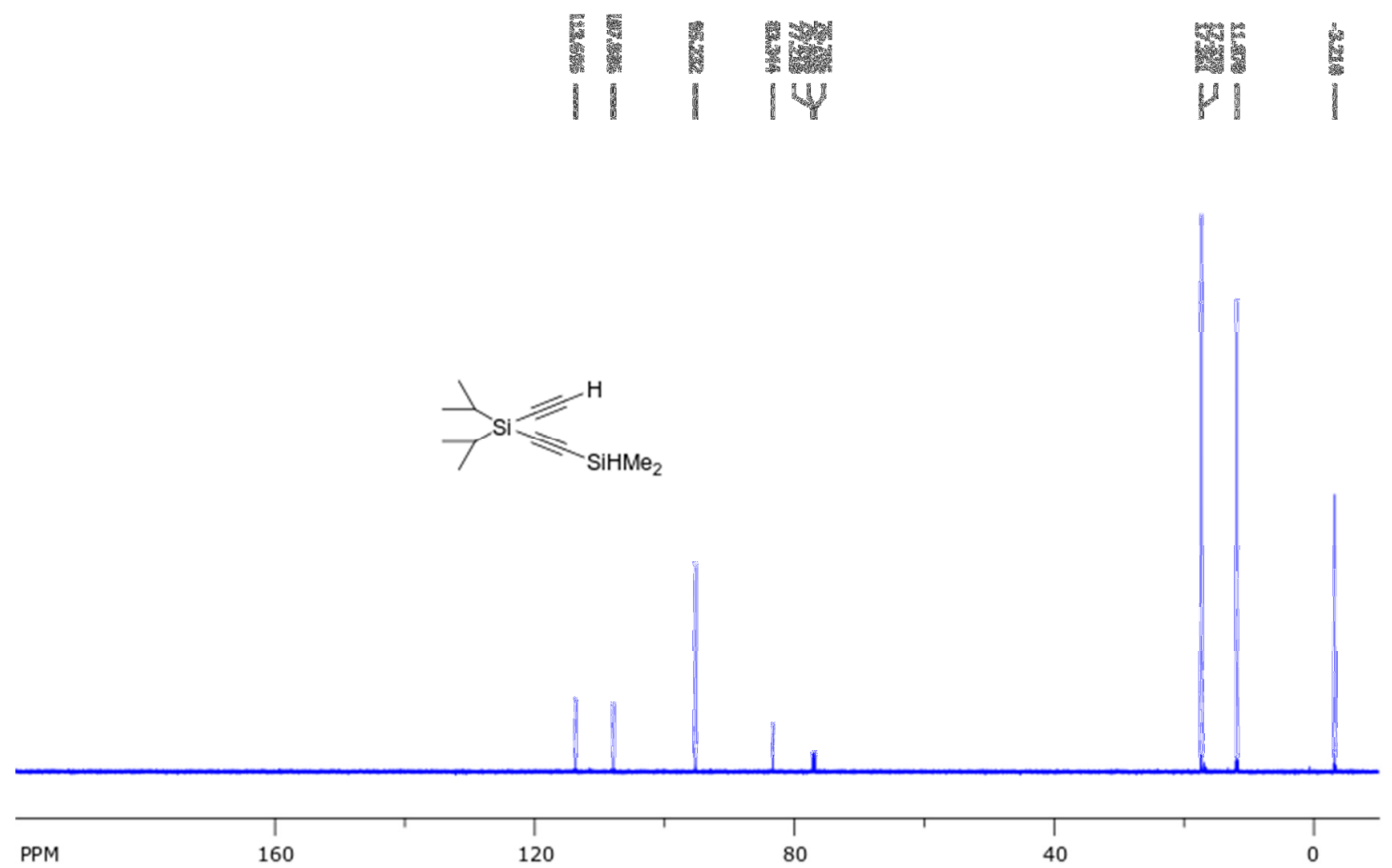

Figure S2. ${ }^{13} \mathrm{C}\left\{{ }^{1} \mathrm{H}\right\}$ NMR spectrum $\left(150.9 \mathrm{MHz}, \mathrm{CDCl}_{3}\right)$ of $\mathbf{1 d}$. 


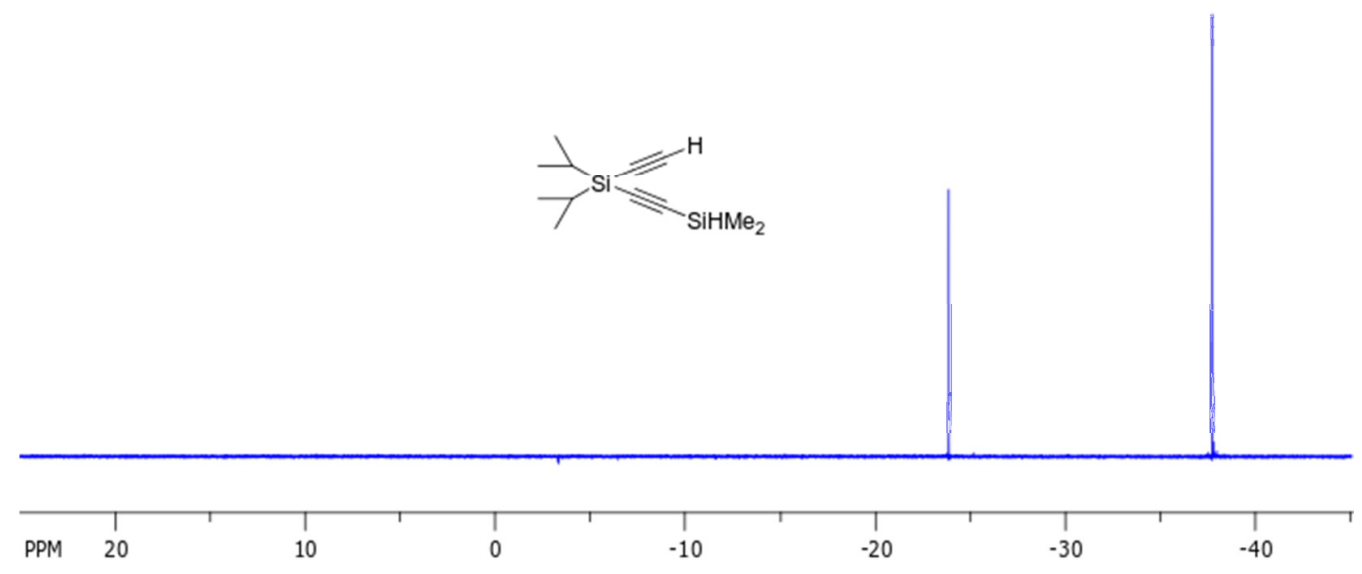

Figure S3. ${ }^{29} \mathrm{Si}\left\{{ }^{1} \mathrm{H}\right\}$ NMR spectrum (refocused INEPT, $119.2 \mathrm{MHz}, \mathrm{CDCl}_{3}$ ) of $\mathbf{1 d}$.

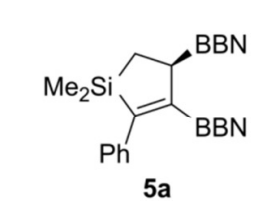

$5 a$
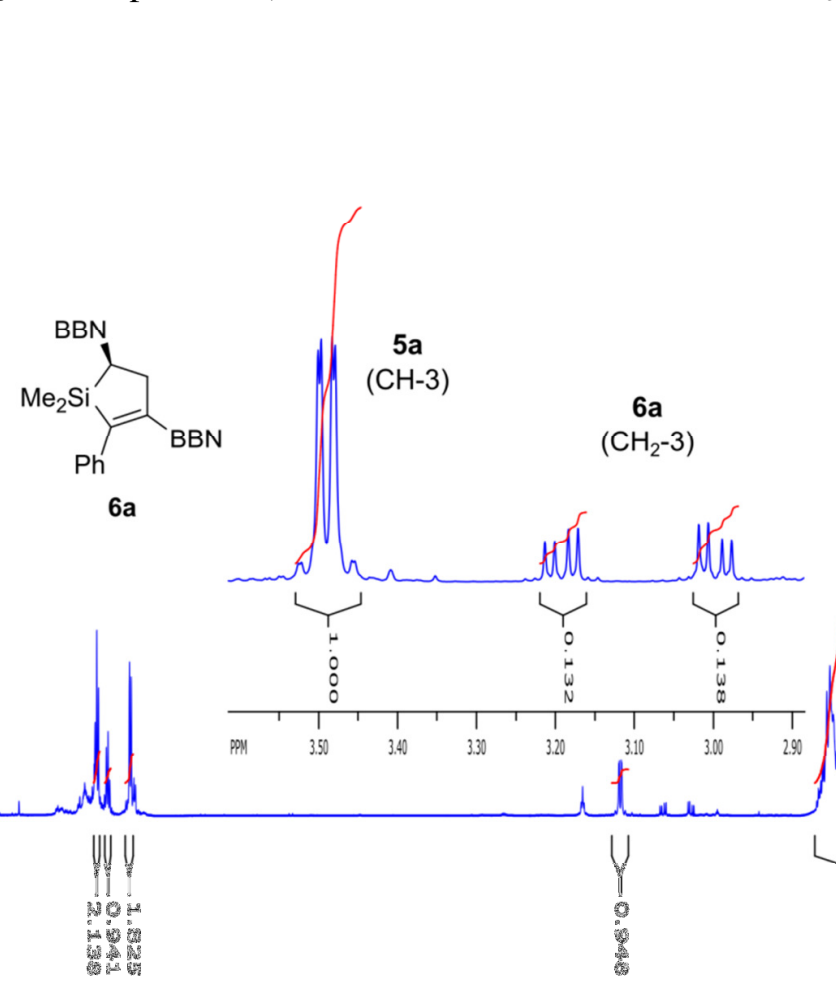

PPM

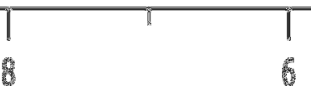

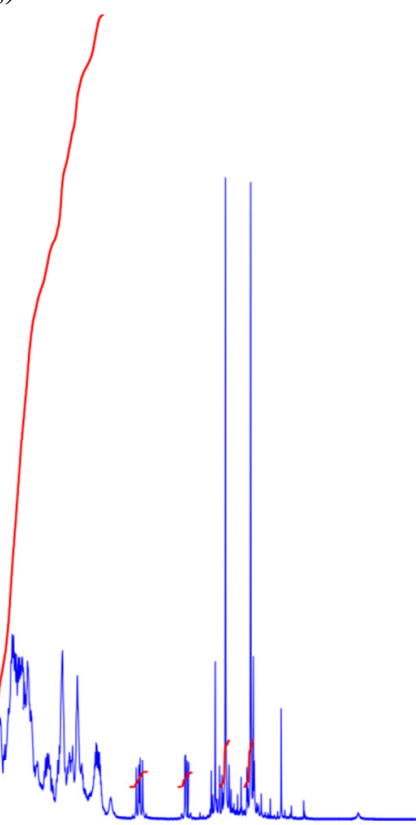
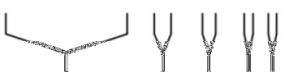

5

8 繁籍

2

Figure S4. ${ }^{1} \mathrm{H}$ NMR spectrum $\left(600 \mathrm{MHz}, \mathrm{C}_{6} \mathrm{D}_{6}\right)$ of the reaction mixture containing $5 \mathbf{a}$ and $6 \mathbf{6}$. 


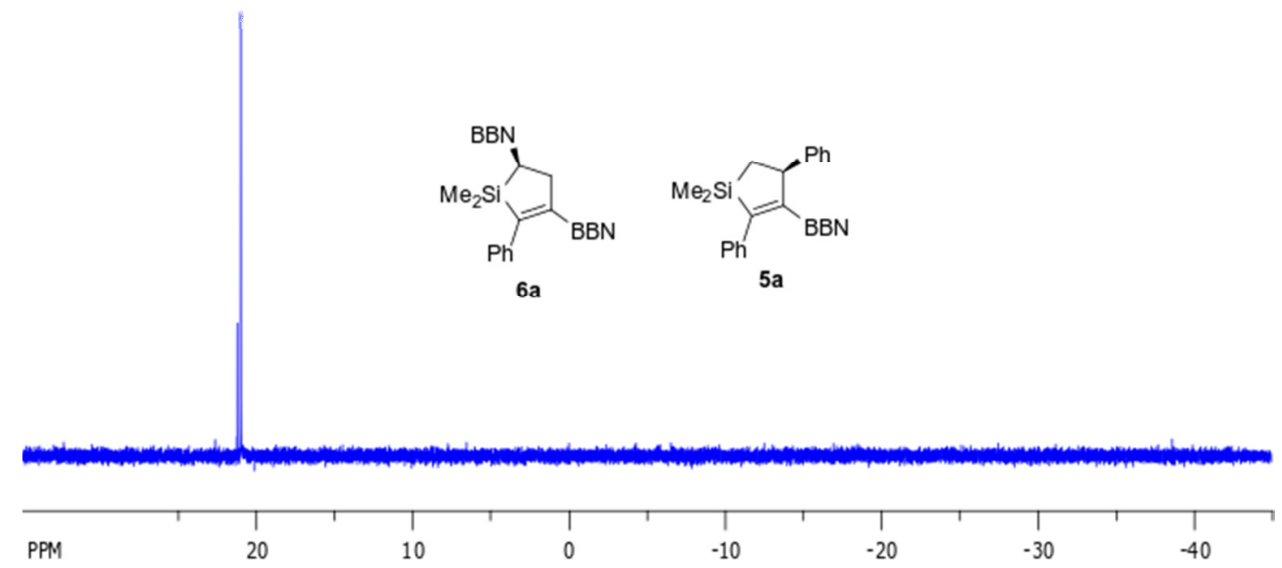

Figure S5. ${ }^{29} \mathrm{Si}\left\{{ }^{1} \mathrm{H}\right\}$ NMR spectrum (refocused INEPT, $119.2 \mathrm{MHz}, \mathrm{C}_{6} \mathrm{D}_{6}$ ) of the reaction mixture containing $5 \mathbf{a}$ and $\mathbf{6 a}$.

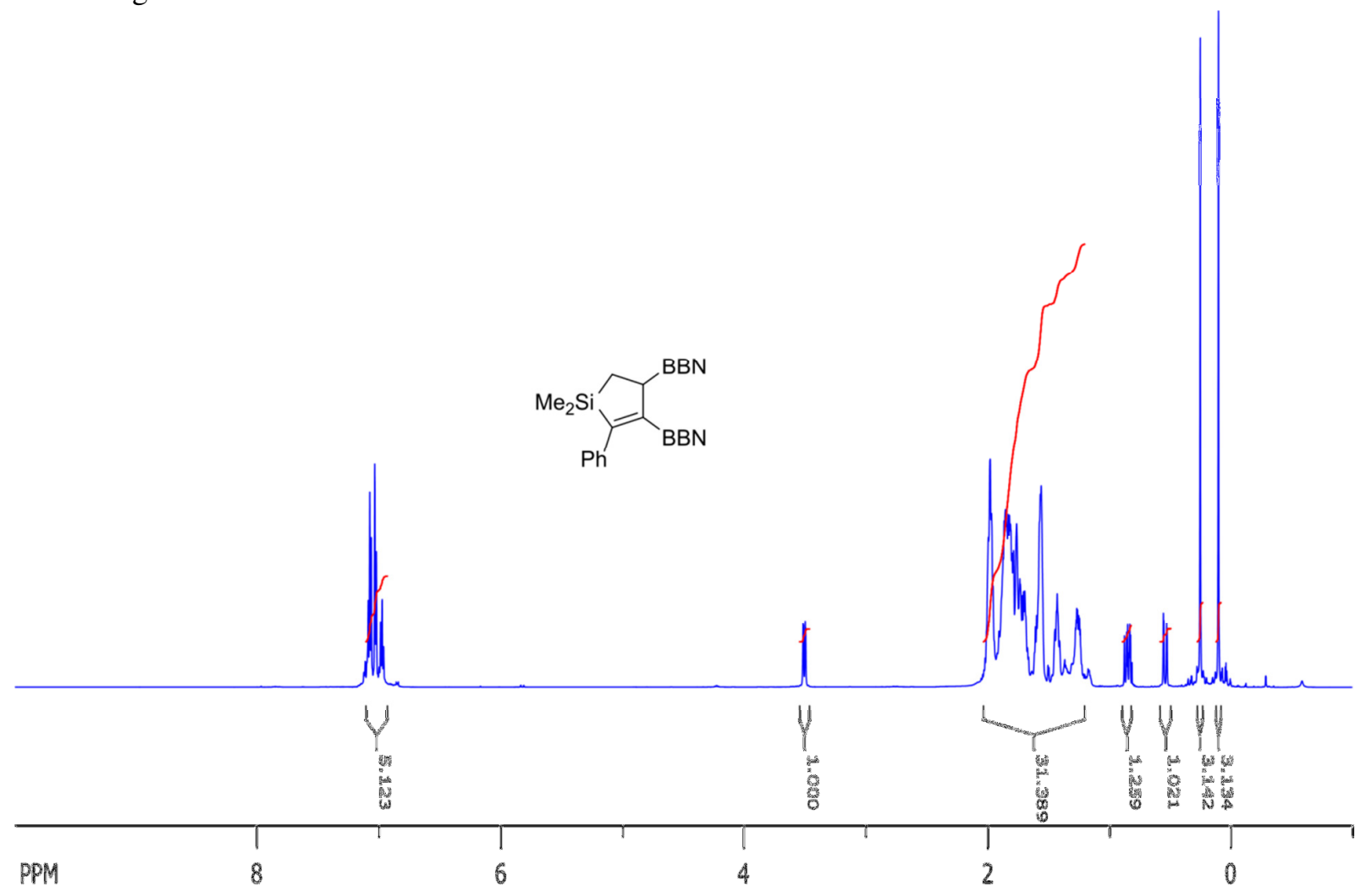

Figure S6. ${ }^{1} \mathrm{H}$ NMR spectrum $\left(600 \mathrm{MHz}, \mathrm{C}_{6} \mathrm{D}_{6}\right)$ of $\mathbf{5 a}$. 


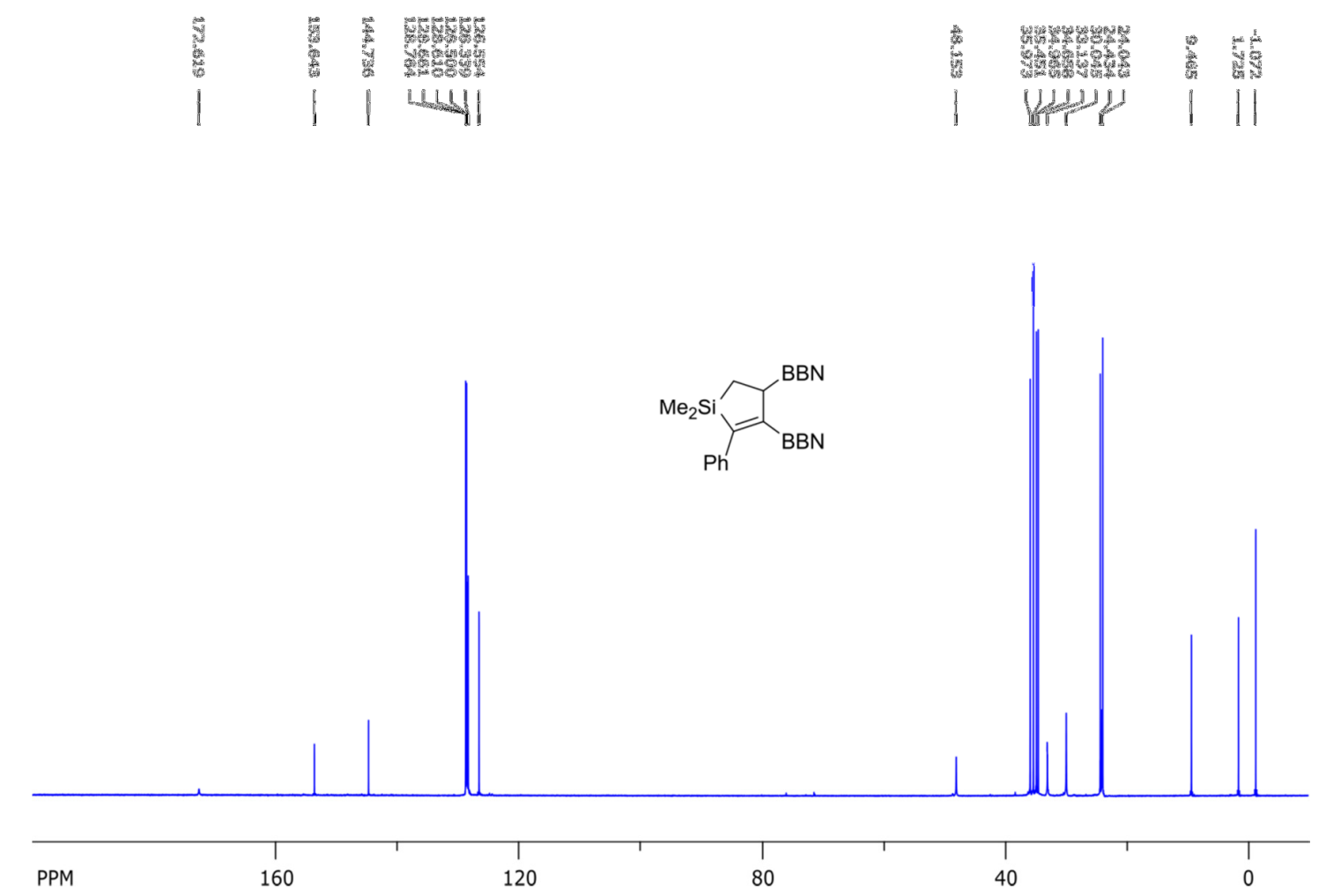

Figure S7. ${ }^{13} \mathrm{C}\left\{{ }^{1} \mathrm{H}\right\}$ NMR spectrum $\left(150.9 \mathrm{MHz}, \mathrm{C}_{6} \mathrm{D}_{6}\right)$ of $\mathbf{5 a}$.
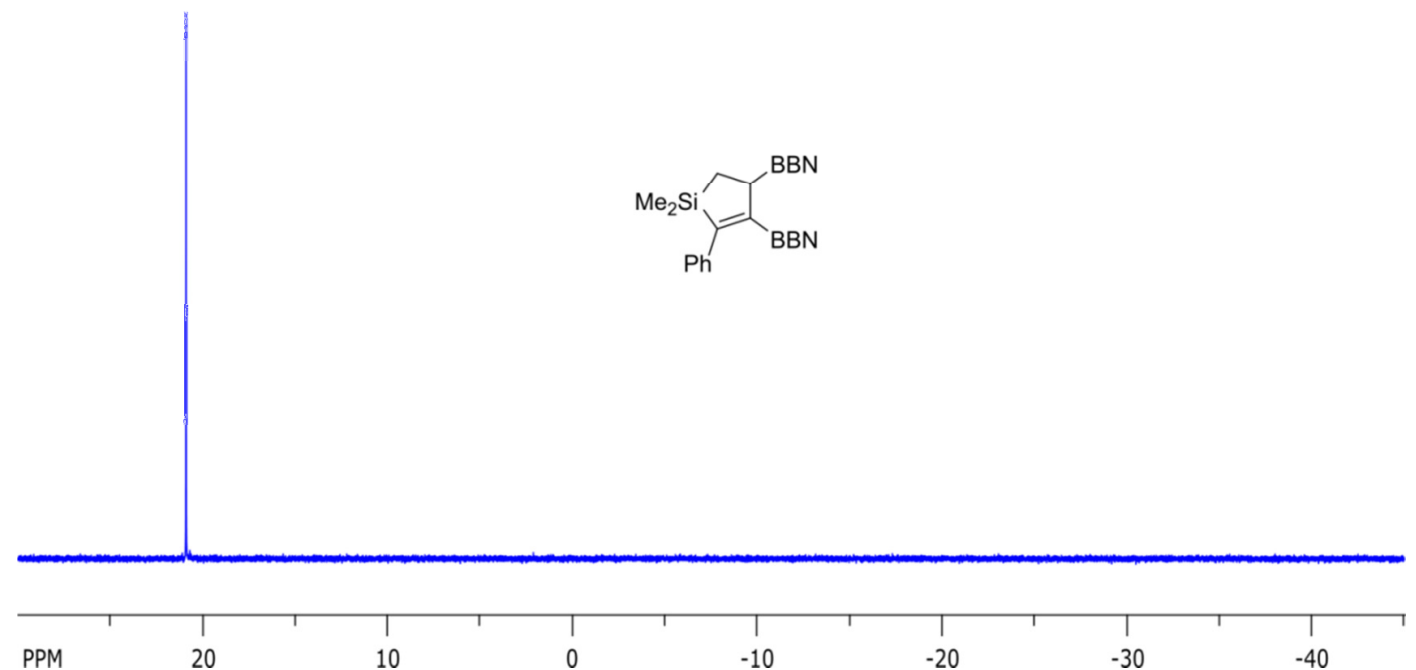

Figure S8. ${ }^{29} \mathrm{Si}\left\{{ }^{1} \mathrm{H}\right\}$ NMR spectrum (refocused INEPT, $119.2 \mathrm{MHz}, \mathrm{C}_{6} \mathrm{D}_{6}$ ) of $\mathbf{5 a}$. 


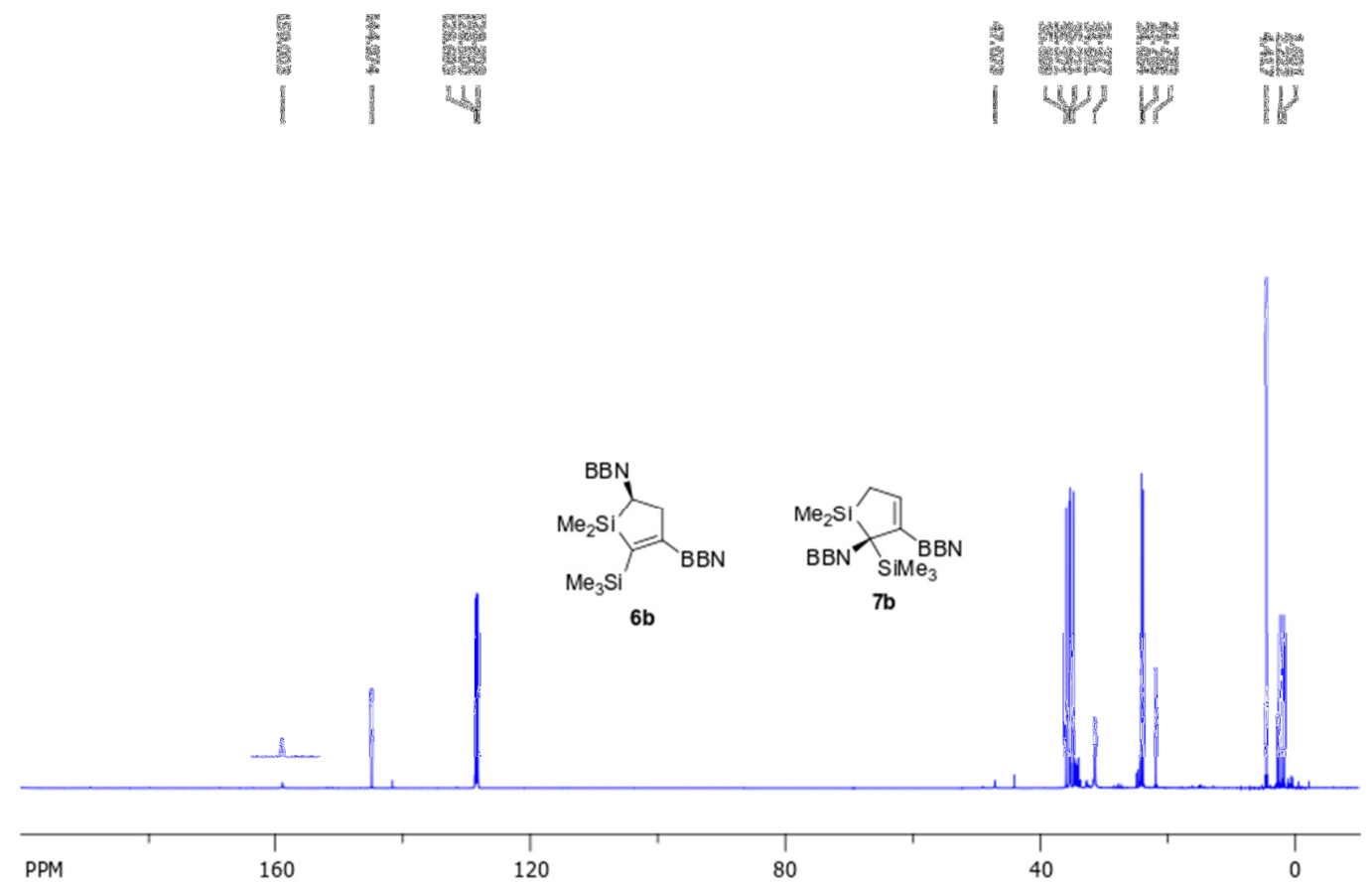

Figure S9. ${ }^{13} \mathrm{C}\left\{{ }^{1} \mathrm{H}\right\}$ NMR spectrum $\left(150.9 \mathrm{MHz}, \mathrm{C}_{6} \mathrm{D}_{6}\right)$ of the reaction mixture containing $\mathbf{6 b}$ and $7 \mathbf{b}$.
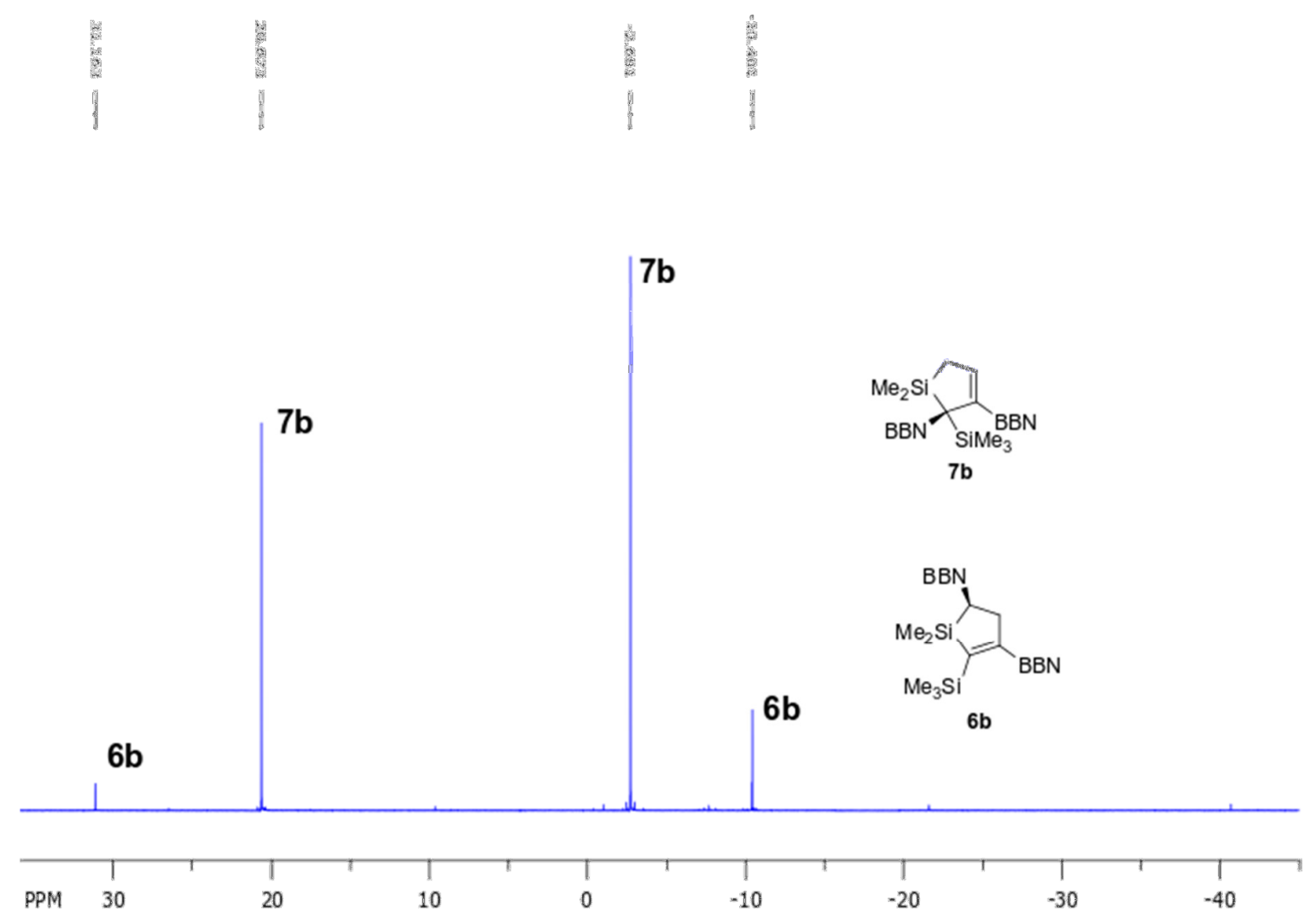

Figure S10. ${ }^{29} \mathrm{Si}\left\{{ }^{1} \mathrm{H}\right\}$ NMR spectrum (refocused INEPT, $119.2 \mathrm{MHz}, \mathrm{C}_{6} \mathrm{D}_{6}$ ) of the reaction mixture containing $6 \mathbf{b}$ and $\mathbf{7 b}$. 


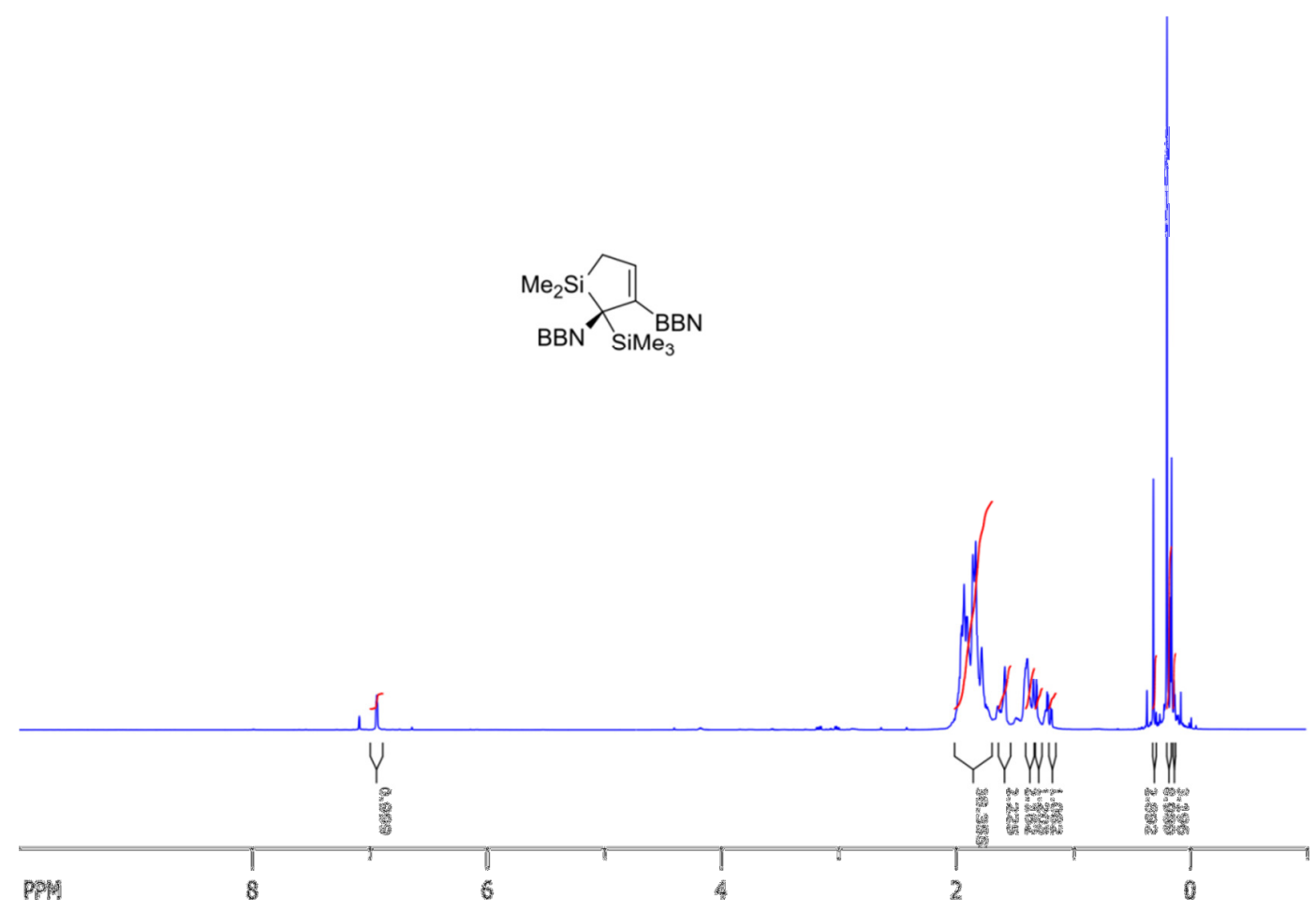

Figure S11. ${ }^{1} \mathrm{H}$ NMR spectrum $\left(600 \mathrm{MHz}, \mathrm{C}_{6} \mathrm{D}_{6}\right)$ of $\mathbf{7 b}$.
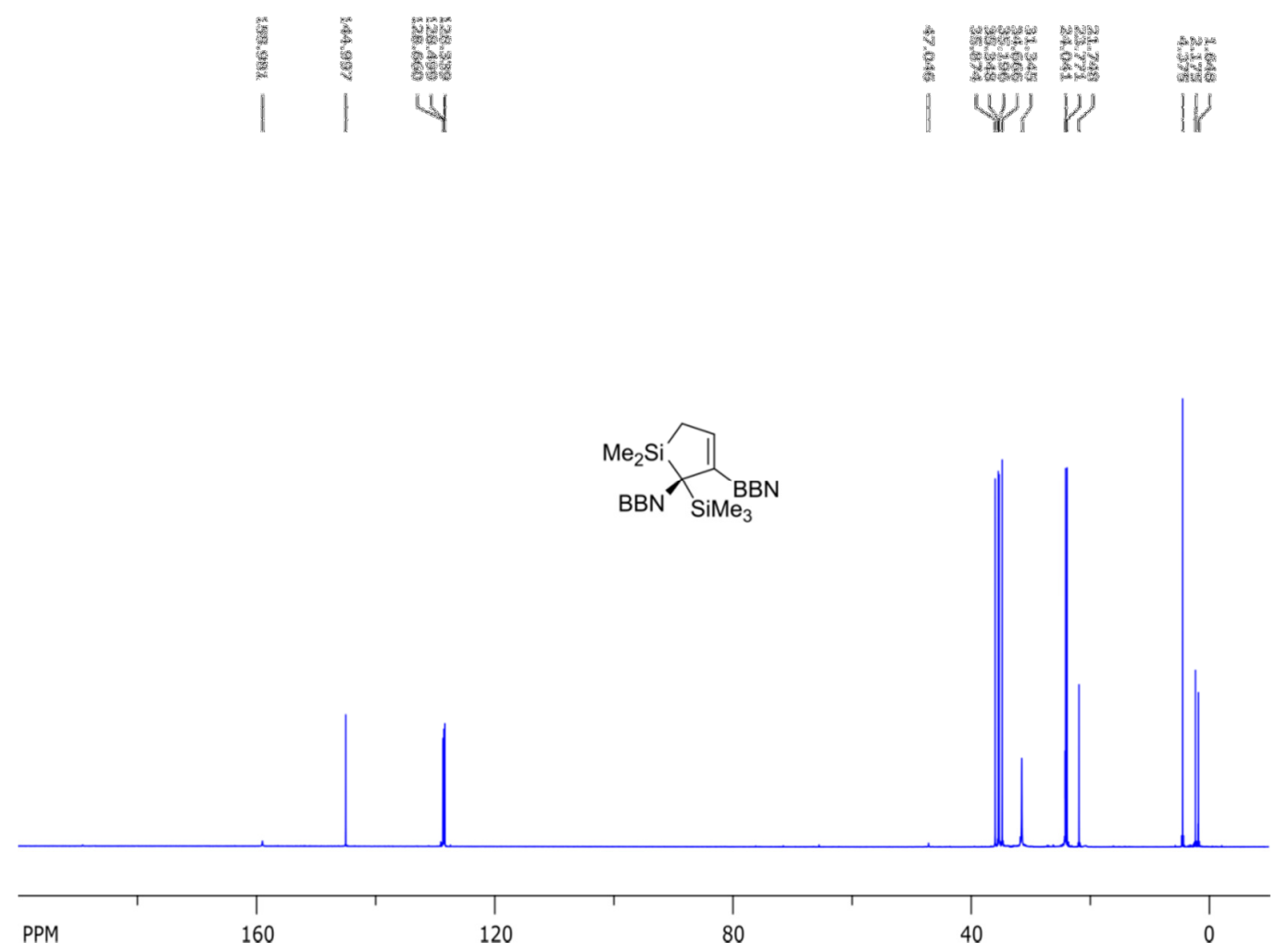

Figure S12. ${ }^{13} \mathrm{C}\left\{{ }^{1} \mathrm{H}\right\}$ NMR spectrum $\left(150.9 \mathrm{MHz}, \mathrm{C}_{6} \mathrm{D}_{6}\right)$ of $\mathbf{7 b}$. 

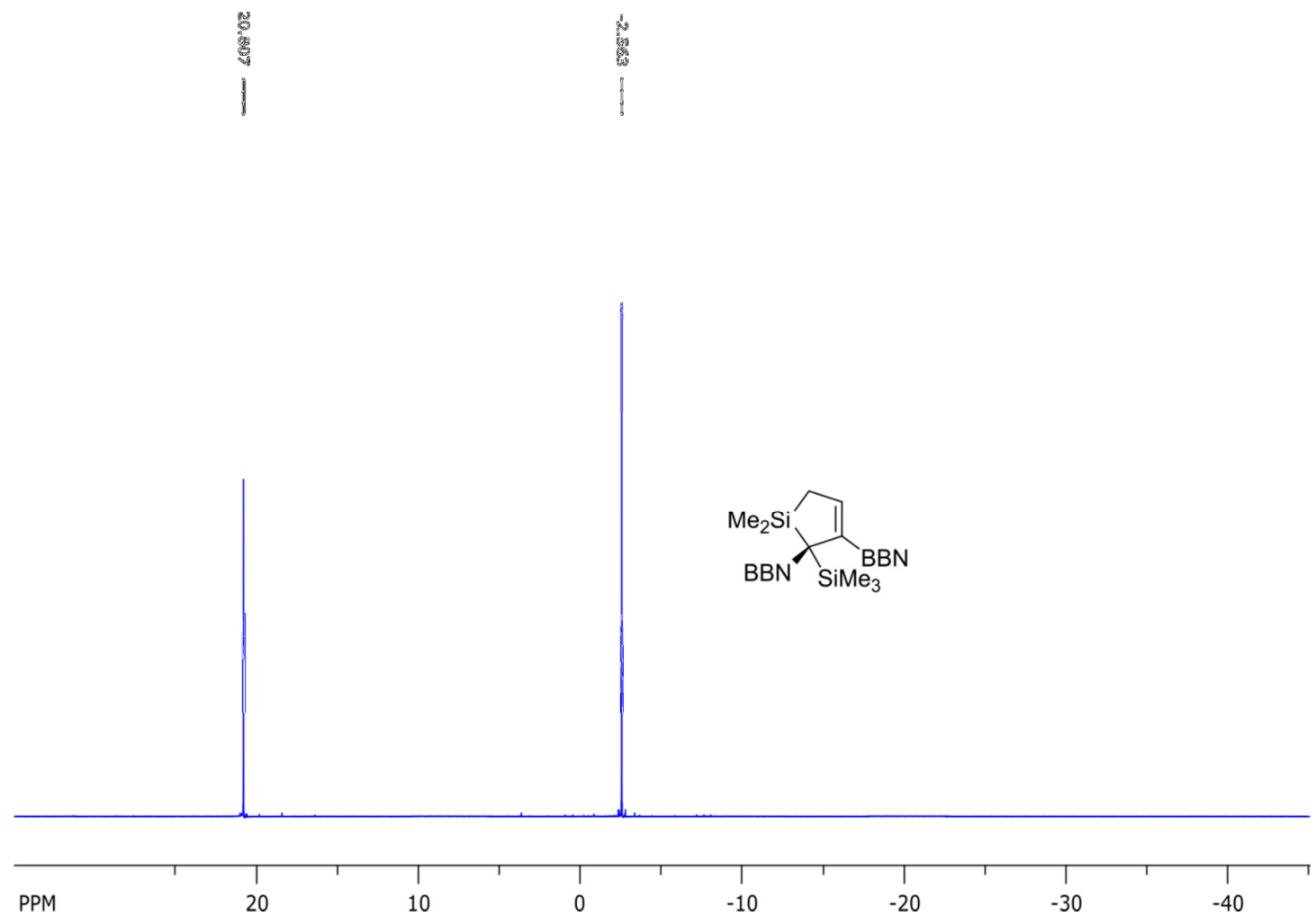

Figure S13. ${ }^{29} \mathrm{Si}\left\{{ }^{1} \mathrm{H}\right\}$ NMR spectrum (refocused INEPT, $119.2 \mathrm{MHz}, \mathrm{C}_{6} \mathrm{D}_{6}$ ) of $\mathbf{7 b}$.
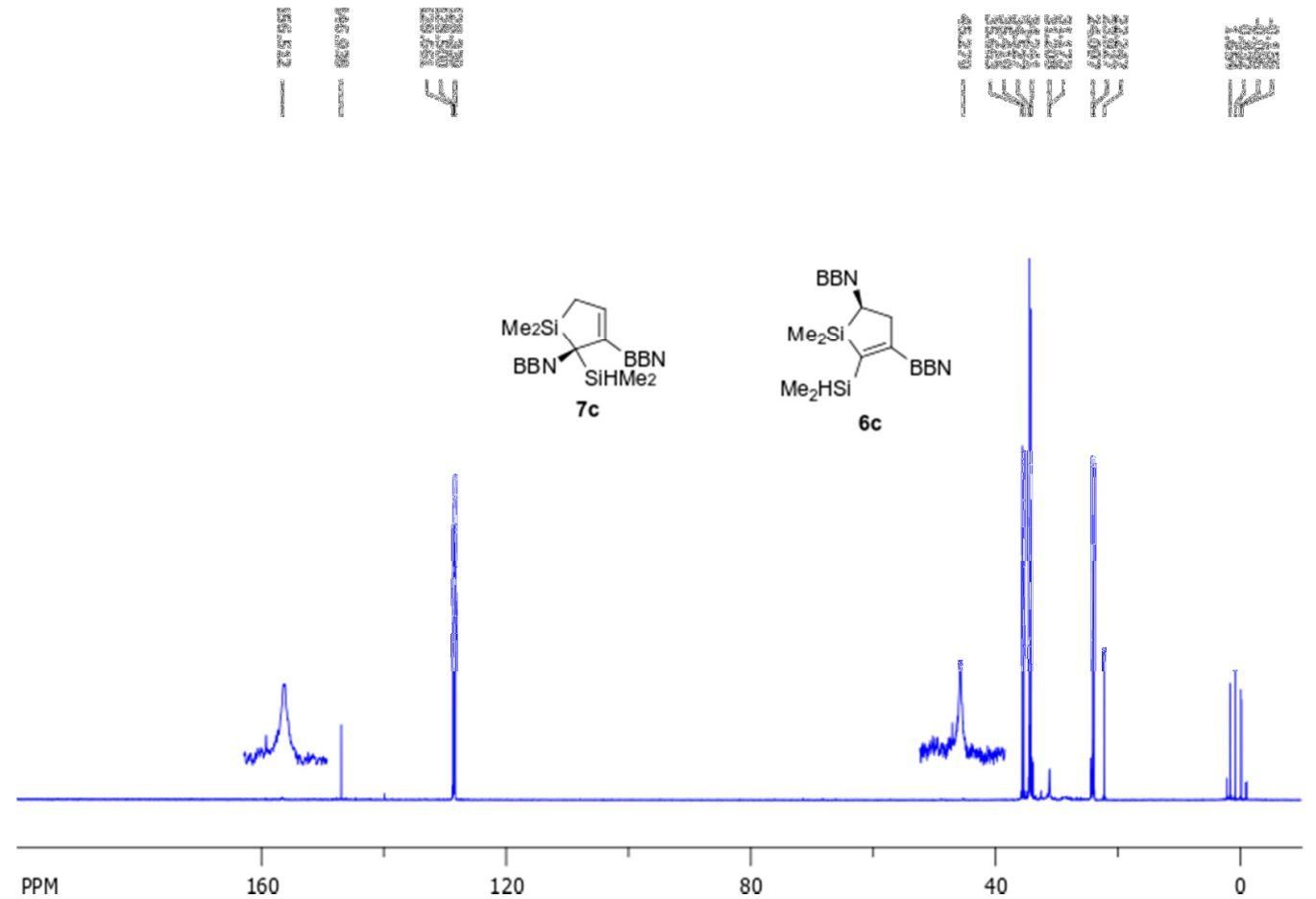

Figure S14. ${ }^{13} \mathrm{C}\left\{{ }^{1} \mathrm{H}\right\}$ NMR spectrum $\left(150.9 \mathrm{MHz}, \mathrm{C}_{6} \mathrm{D}_{6}\right)$ of the reaction mixture containing $\mathbf{6 c}$ and $\mathbf{7 c}$. 


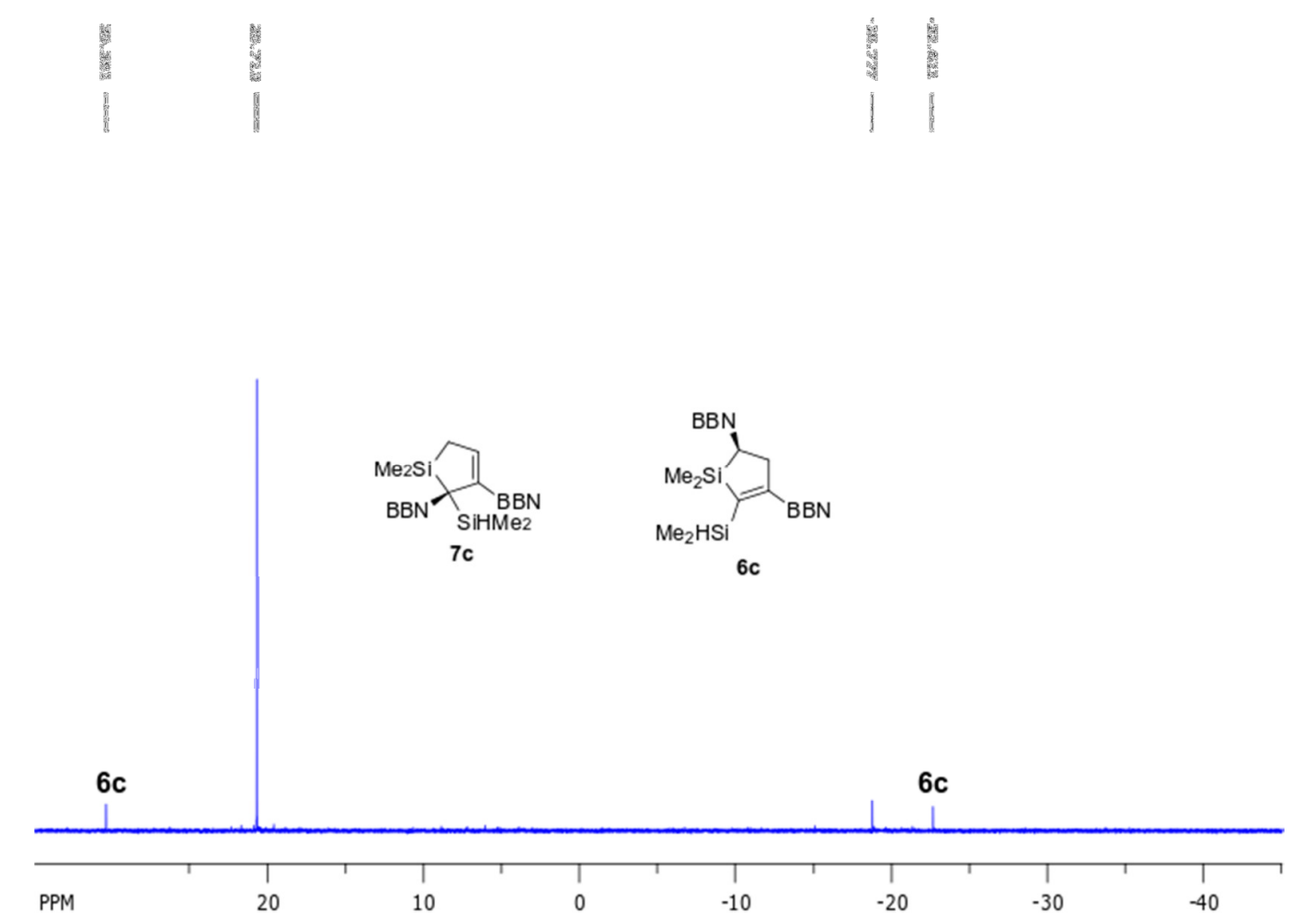

Figure S15. ${ }^{29} \mathrm{Si}\left\{{ }^{1} \mathrm{H}\right\}$ NMR spectrum (refocused INEPT, $119.2 \mathrm{MHz}, \mathrm{C}_{6} \mathrm{D}_{6}$ ) of the reaction mixture containing $\mathbf{6 c}$ and $\mathbf{7 c}$.

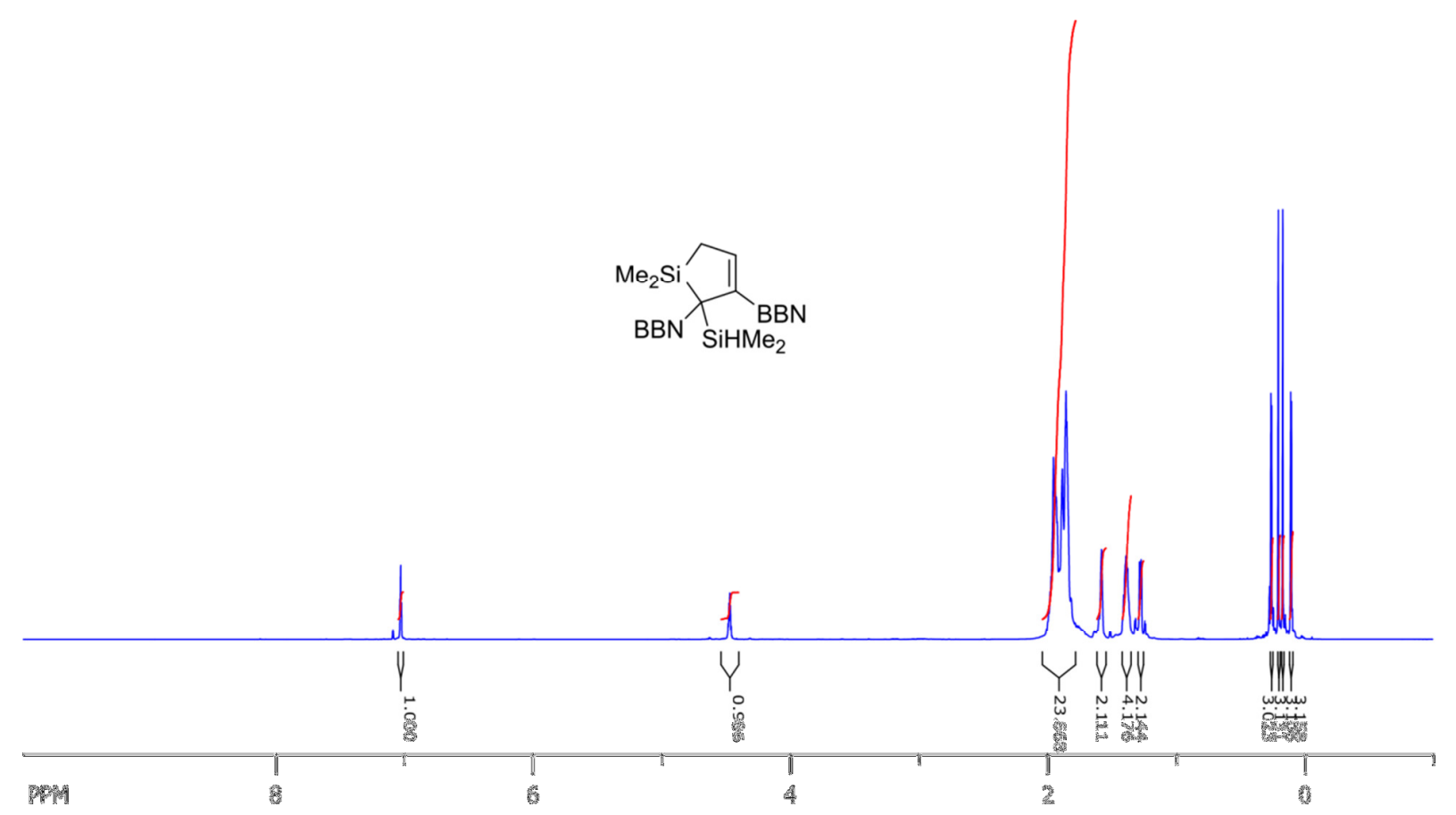

Figure S16. ${ }^{1} \mathrm{H}$ NMR spectrum $\left(600 \mathrm{MHz}, \mathrm{C}_{6} \mathrm{D}_{6}\right)$ of 7 c. 


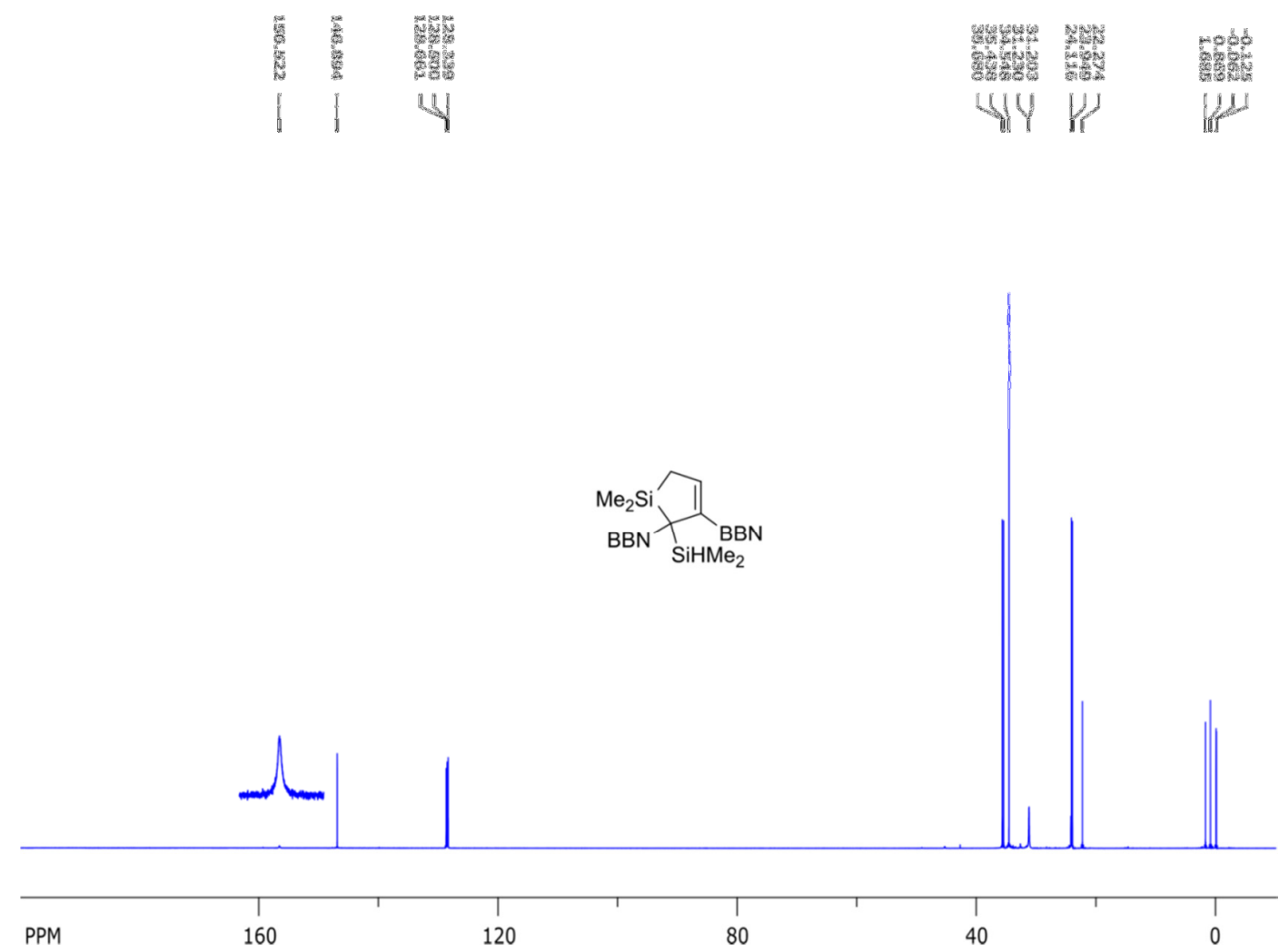

Figure S17. ${ }^{13} \mathrm{C}\left\{{ }^{1} \mathrm{H}\right\}$ NMR spectrum $\left(150.9 \mathrm{MHz}, \mathrm{C}_{6} \mathrm{D}_{6}\right)$ of $7 \mathrm{c}$.

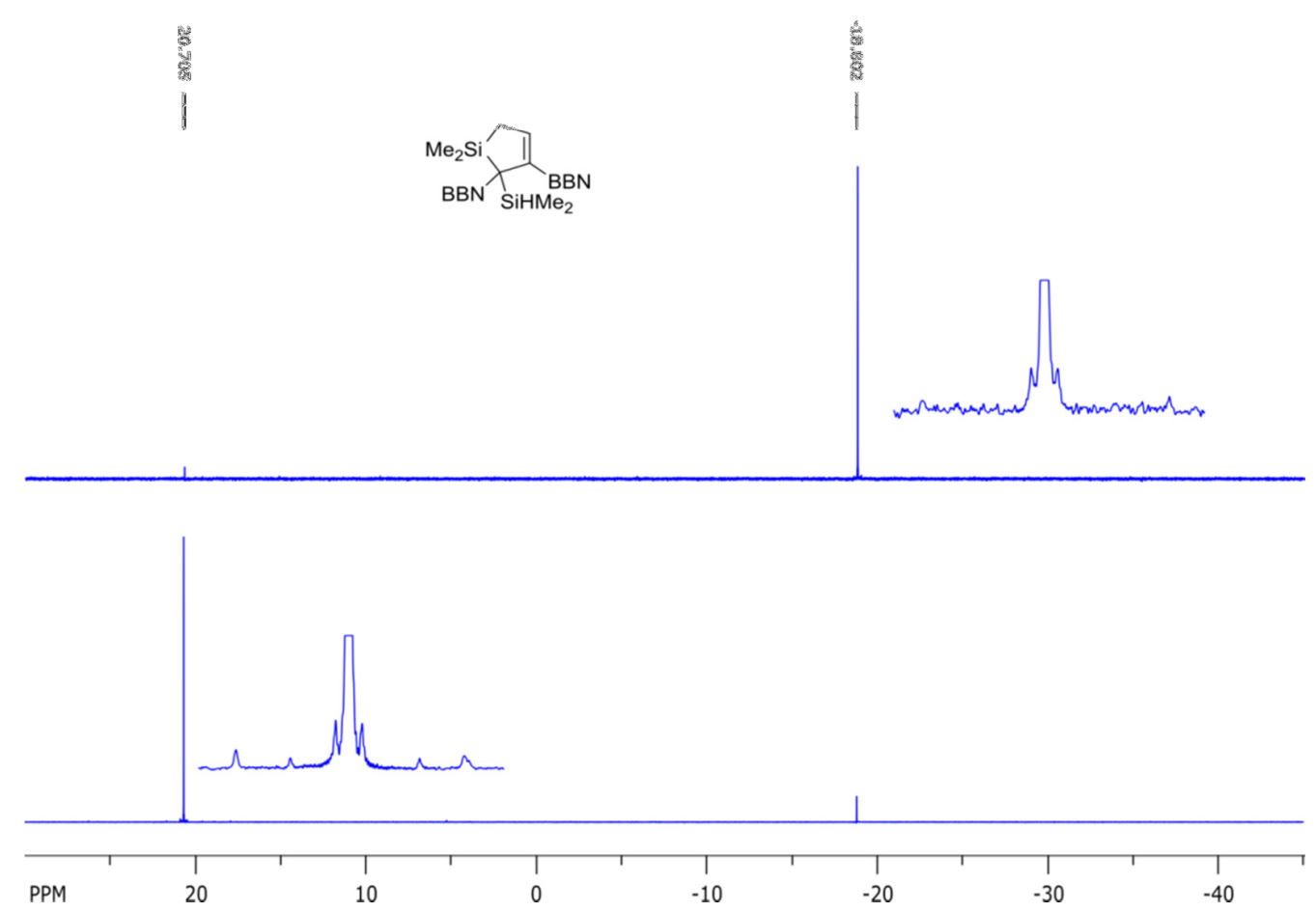

Figure S18. ${ }^{29} \mathrm{Si}\left\{{ }^{1} \mathrm{H}\right\}$ NMR spectrum (refocused INEPT, $119.2 \mathrm{MHz}, \mathrm{C}_{6} \mathrm{D}_{6}$ ) of 7c. Top- INEPT based on ${ }^{1} J\left({ }^{29} \mathrm{Si},{ }^{1} \mathrm{H}\right)=190 \mathrm{~Hz}$, bottom - INEPT based on ${ }^{2} J\left({ }^{29} \mathrm{Si},{ }^{1} \mathrm{H}\right)=7 \mathrm{~Hz}$. 


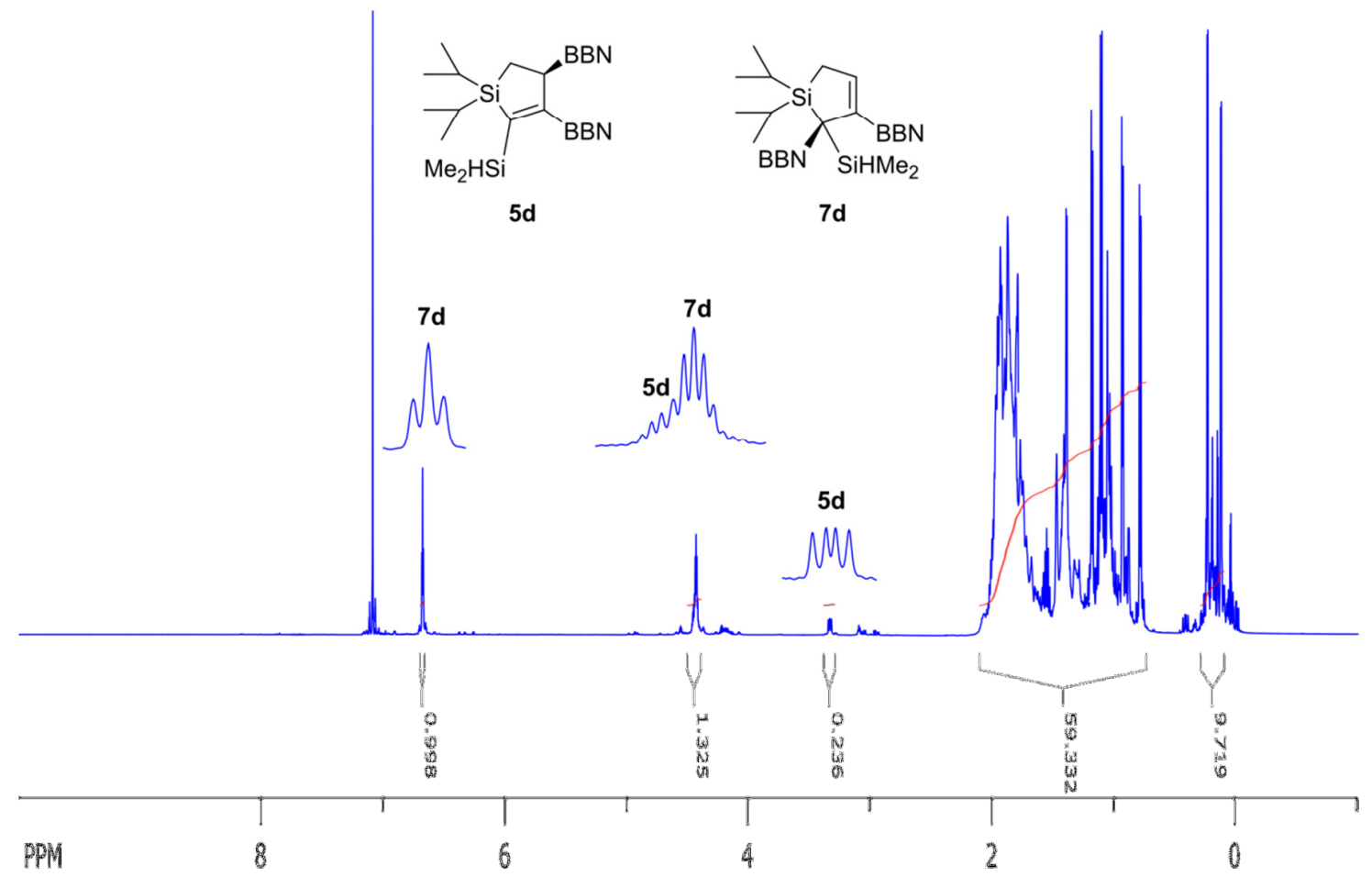

Figure S19. ${ }^{1} \mathrm{H}$ NMR spectrum $\left(600 \mathrm{MHz}, \mathrm{C}_{6} \mathrm{D}_{6}\right)$ of the reaction mixture containing $\mathbf{5 d}, \mathbf{6 d}$ and $7 \mathbf{d}$
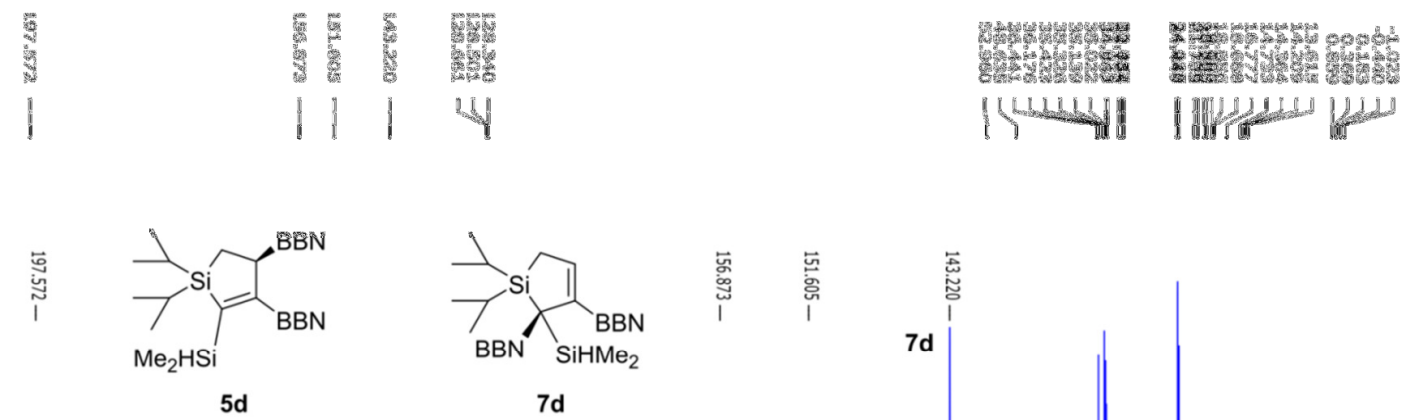

5d

$7 d$

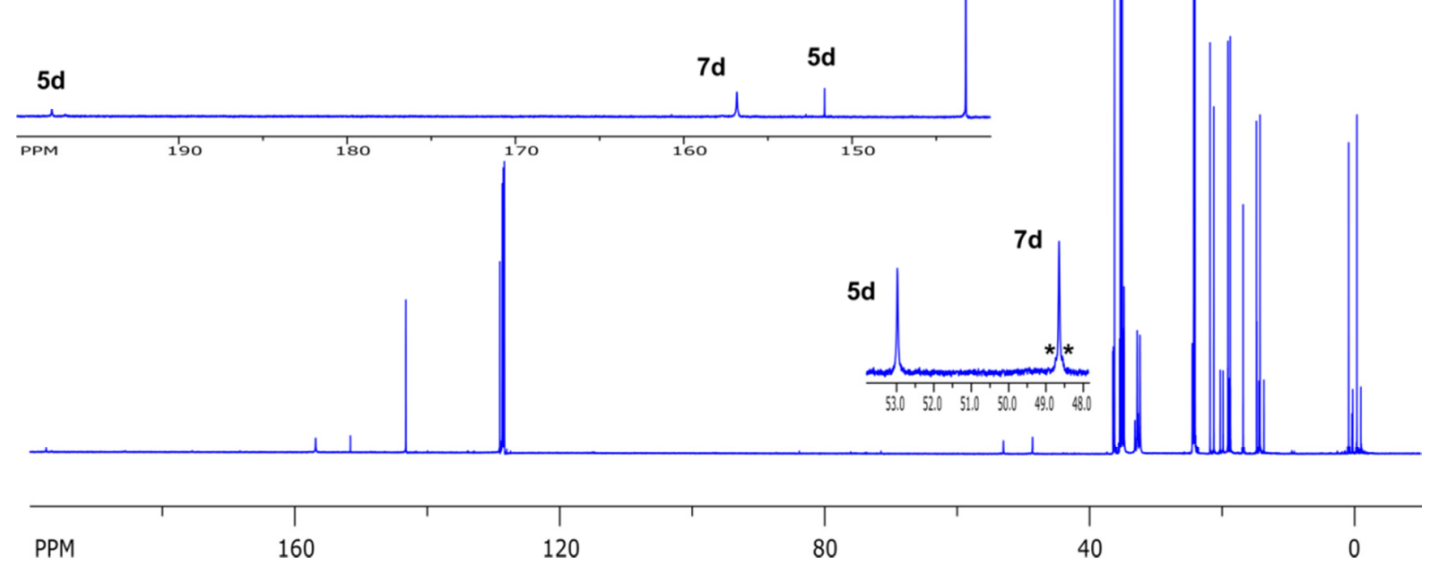

Figure S20. ${ }^{13} \mathrm{C}\left\{{ }^{1} \mathrm{H}\right\}$ NMR spectrum $\left(150.9 \mathrm{MHz}, \mathrm{C}_{6} \mathrm{D}_{6}\right)$ of the equilibrating mixture of $\mathbf{5 d}$ and $\mathbf{7 d}$. 


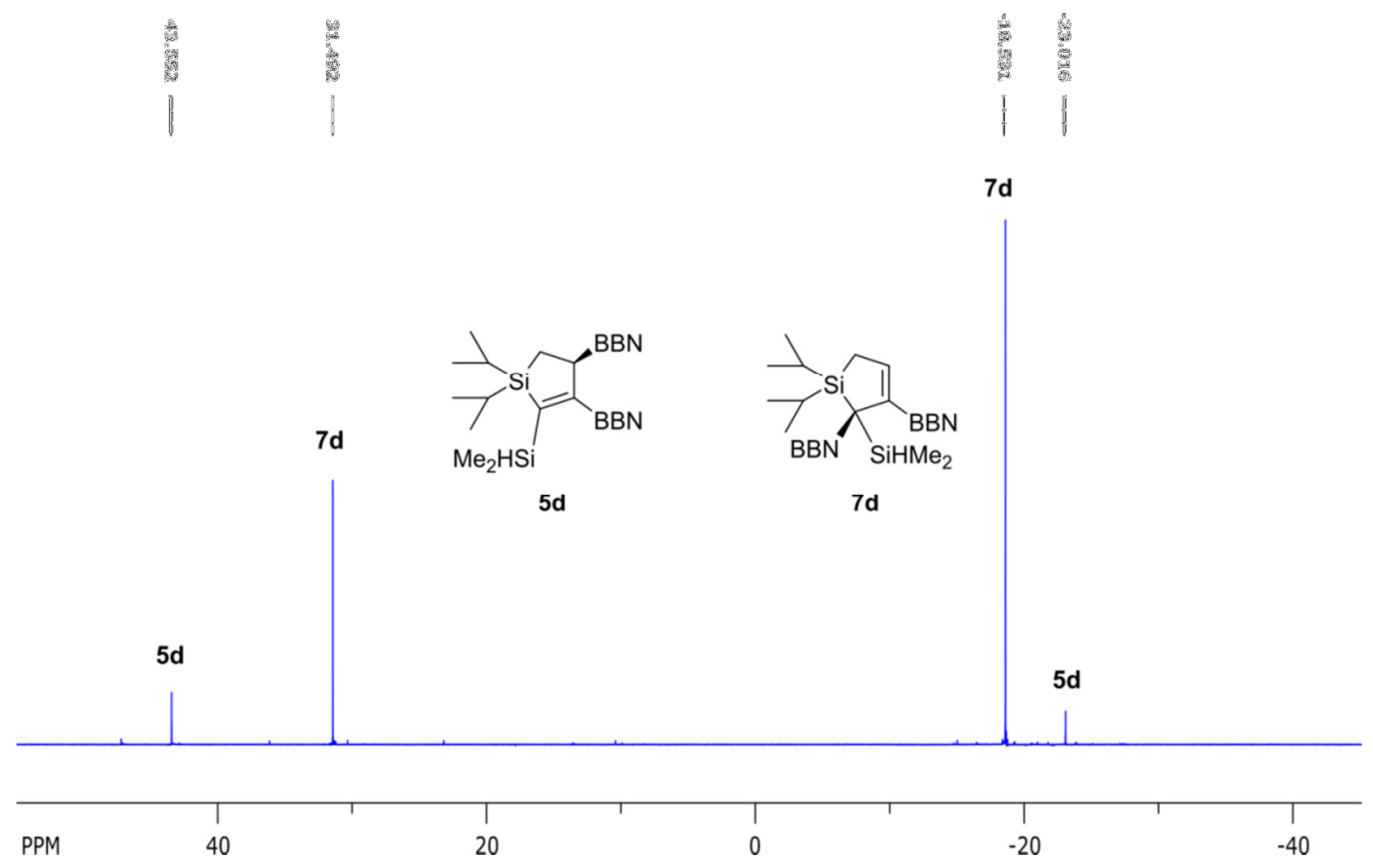

Figure S21. ${ }^{29} \mathrm{Si}\left\{{ }^{1} \mathrm{H}\right\}$ NMR spectrum (refocused INEPT, $119.2 \mathrm{MHz}, \mathrm{C}_{6} \mathrm{D}_{6}$ ) of the equilibrating mixture of $5 d$ and $7 d$.

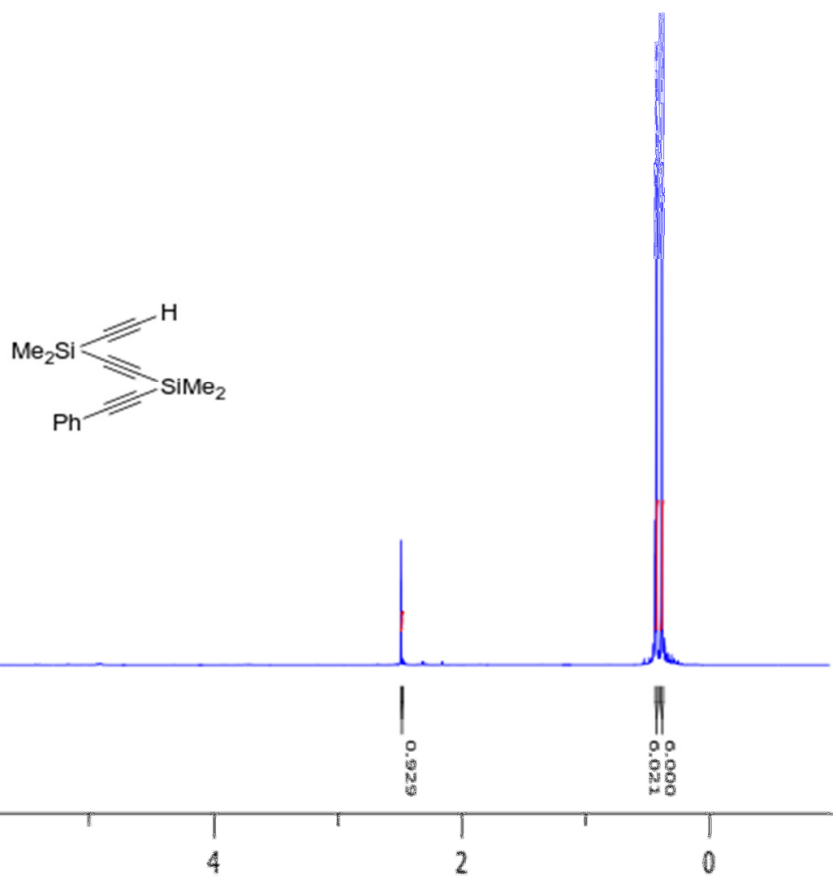

Figure S22. ${ }^{1} \mathrm{H}$ NMR spectrum $\left(600 \mathrm{MHz}, \mathrm{CDCl}_{3}\right)$ of $\mathbf{8 a}$. 


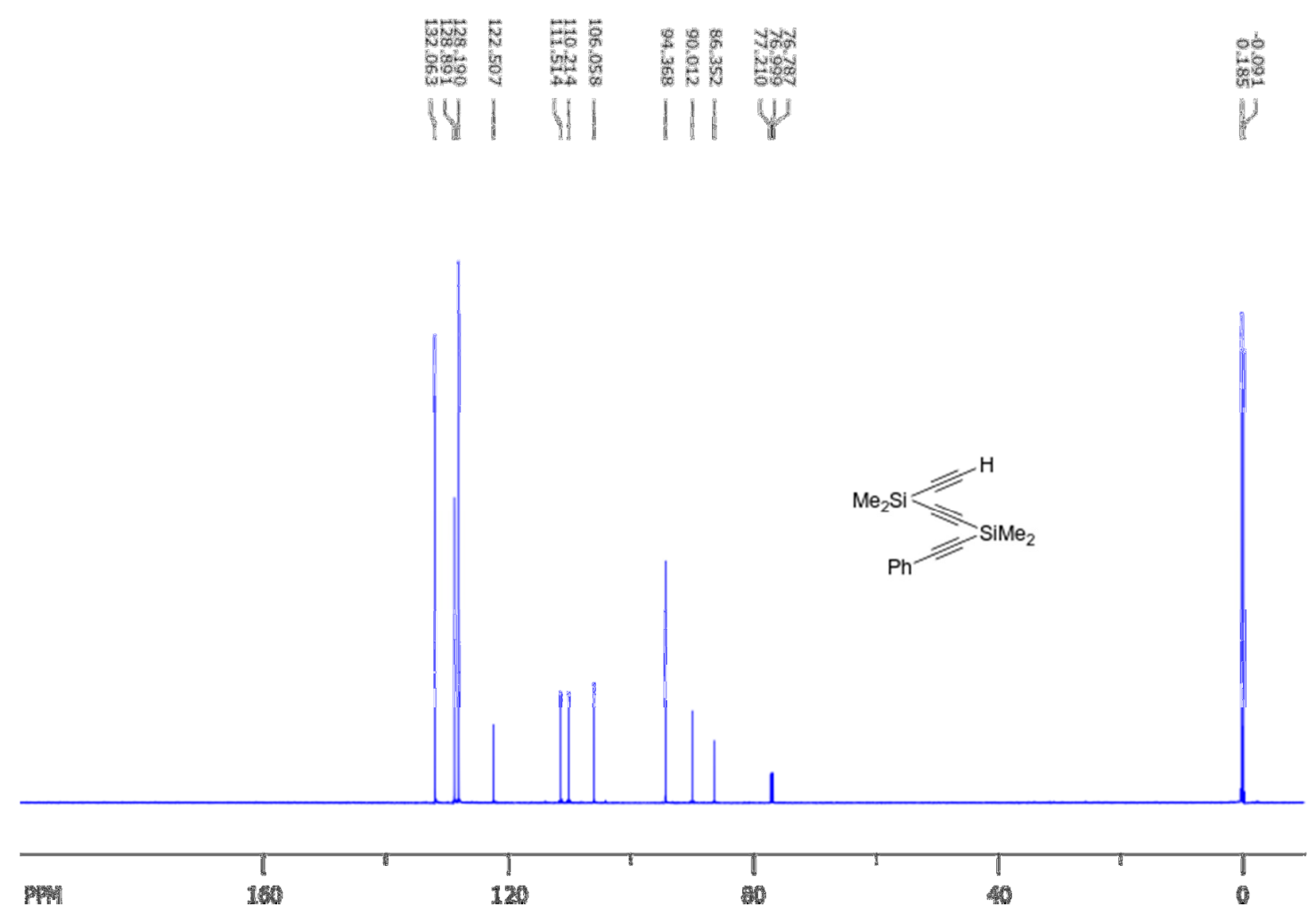

Figure S23. ${ }^{13} \mathrm{C}\left\{{ }^{1} \mathrm{H}\right\}$ NMR spectrum $\left(150.9 \mathrm{MHz}, \mathrm{CDCl}_{3}\right)$ of $\mathbf{8 a}$.

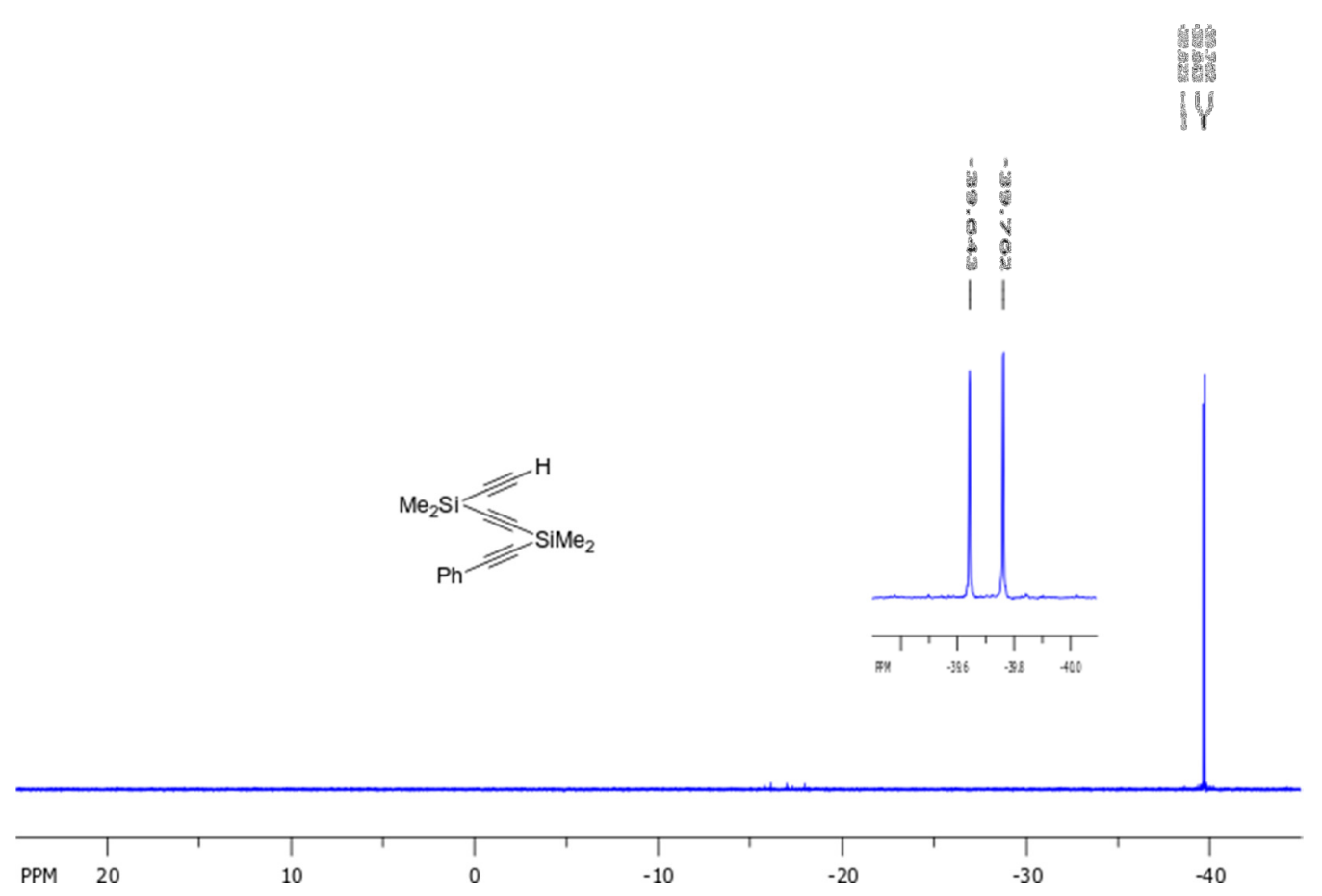

Figure S24. ${ }^{29} \mathrm{Si}\left\{{ }^{1} \mathrm{H}\right\}$ NMR spectrum (refocused INEPT, $119.2 \mathrm{MHz}, \mathrm{CDCl}_{3}$ ) of $\mathbf{8 a}$. 


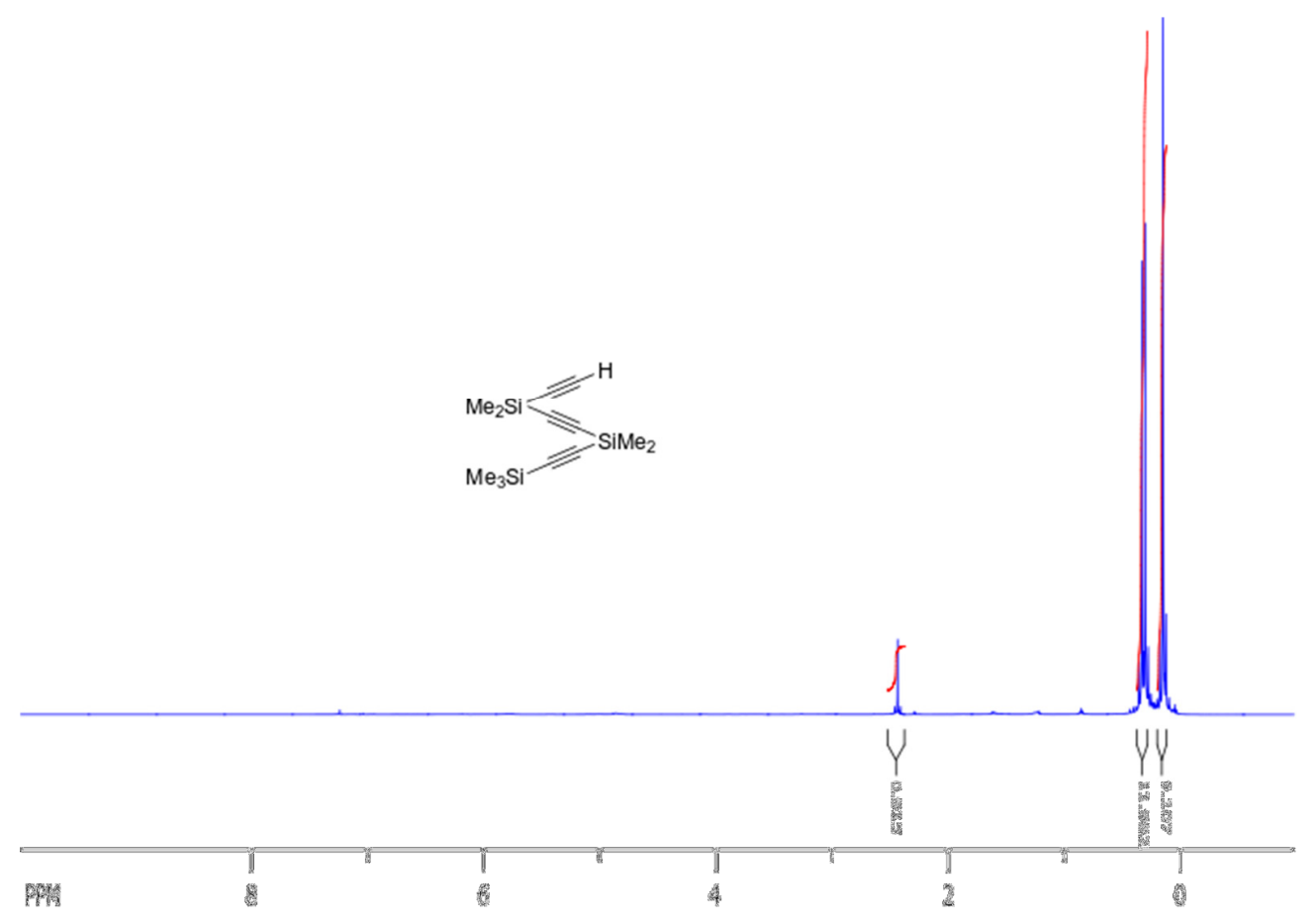

Figure S25. ${ }^{1} \mathrm{H}$ NMR spectrum $\left(600 \mathrm{MHz}, \mathrm{CDCl}_{3}\right)$ of $\mathbf{8 b}$.
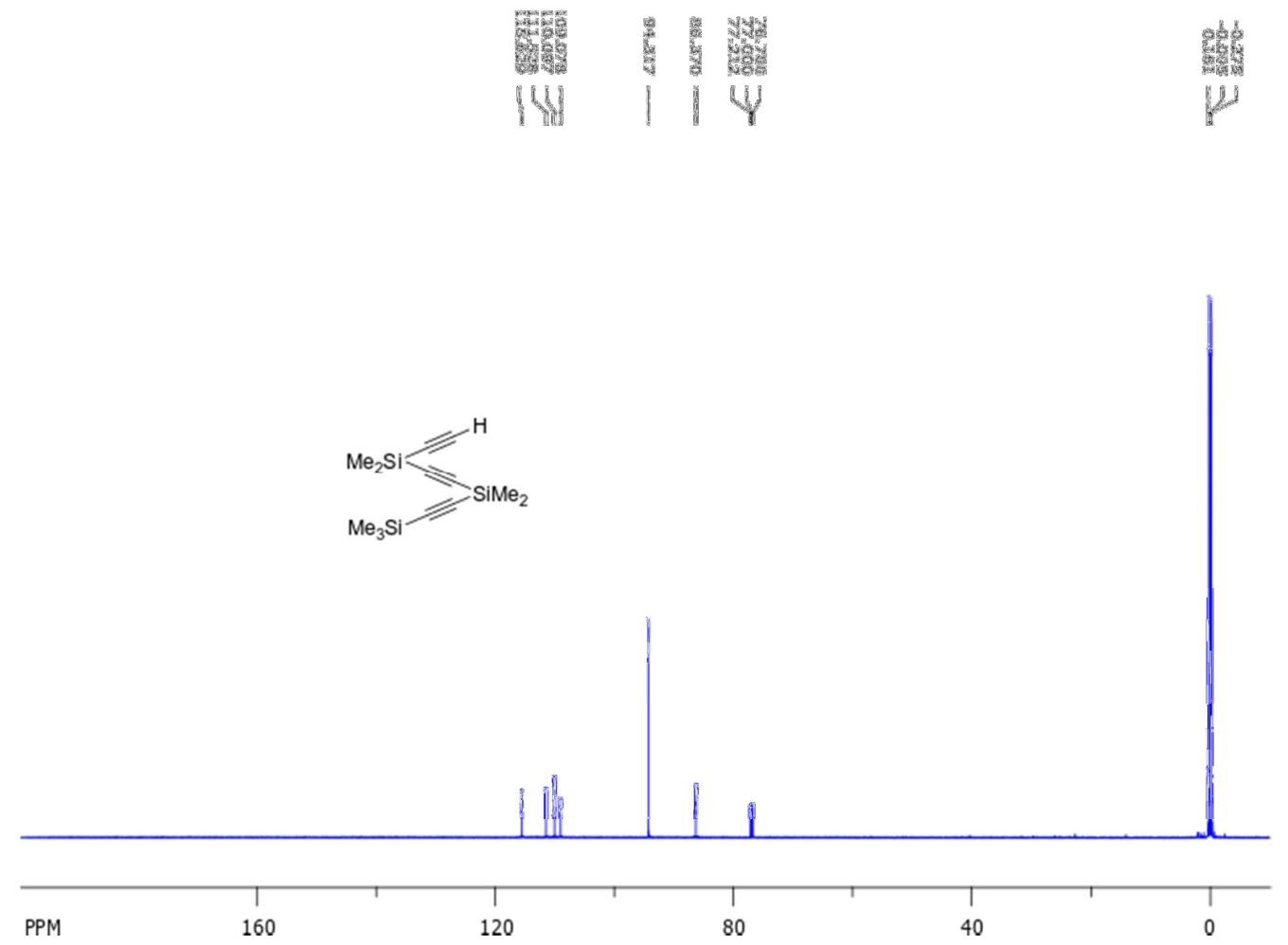

Figure S26. ${ }^{13} \mathrm{C}\left\{{ }^{1} \mathrm{H}\right\}$ NMR spectrum $\left(150.9 \mathrm{MHz}, \mathrm{CDCl}_{3}\right)$ of $\mathbf{8 b}$. 


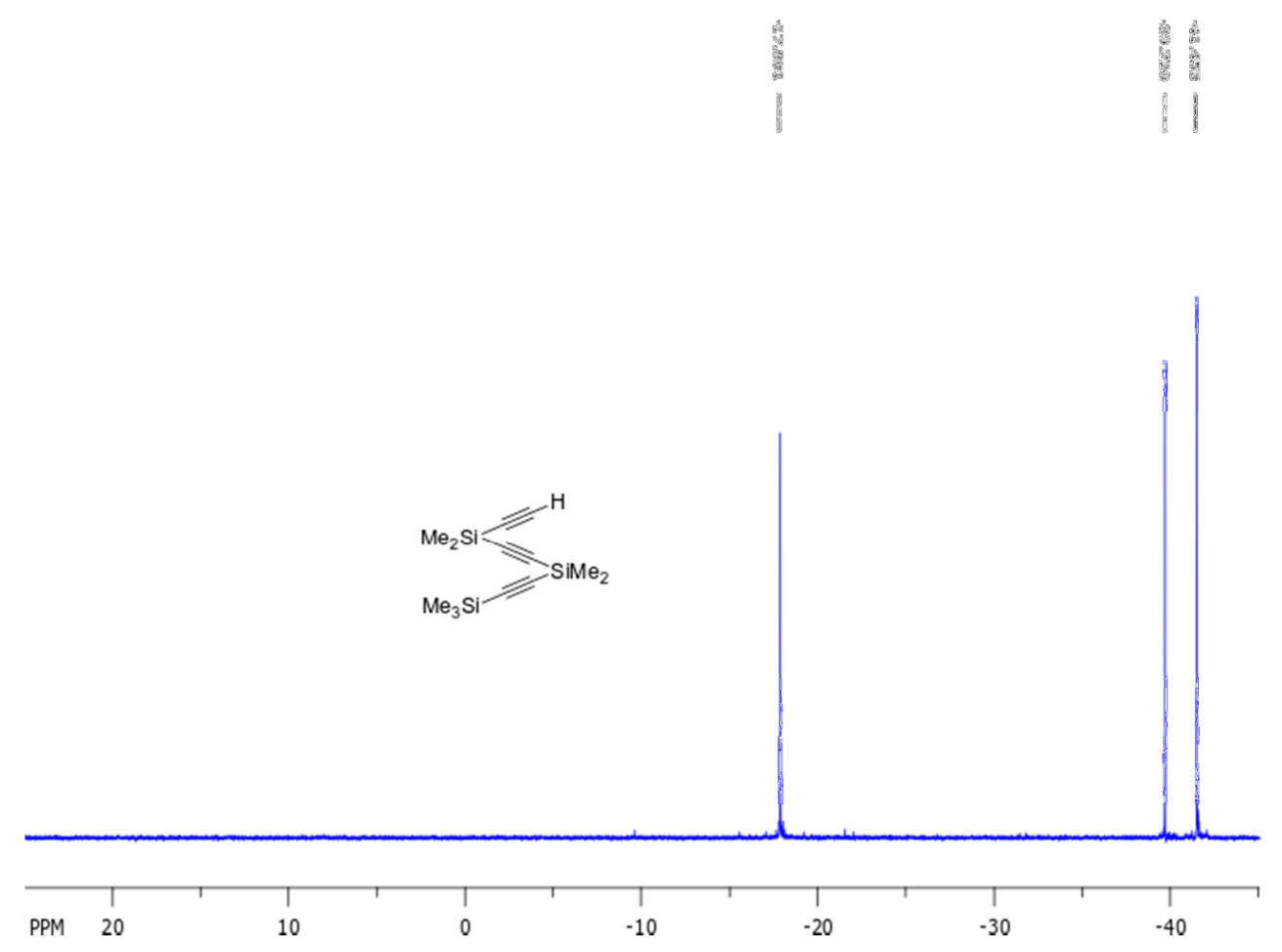

Figure S27. ${ }^{29} \mathrm{Si}\left\{{ }^{1} \mathrm{H}\right\}$ NMR spectrum (refocused INEPT, $119.2 \mathrm{MHz}, \mathrm{CDCl}_{3}$ ) of $\mathbf{8 b}$.

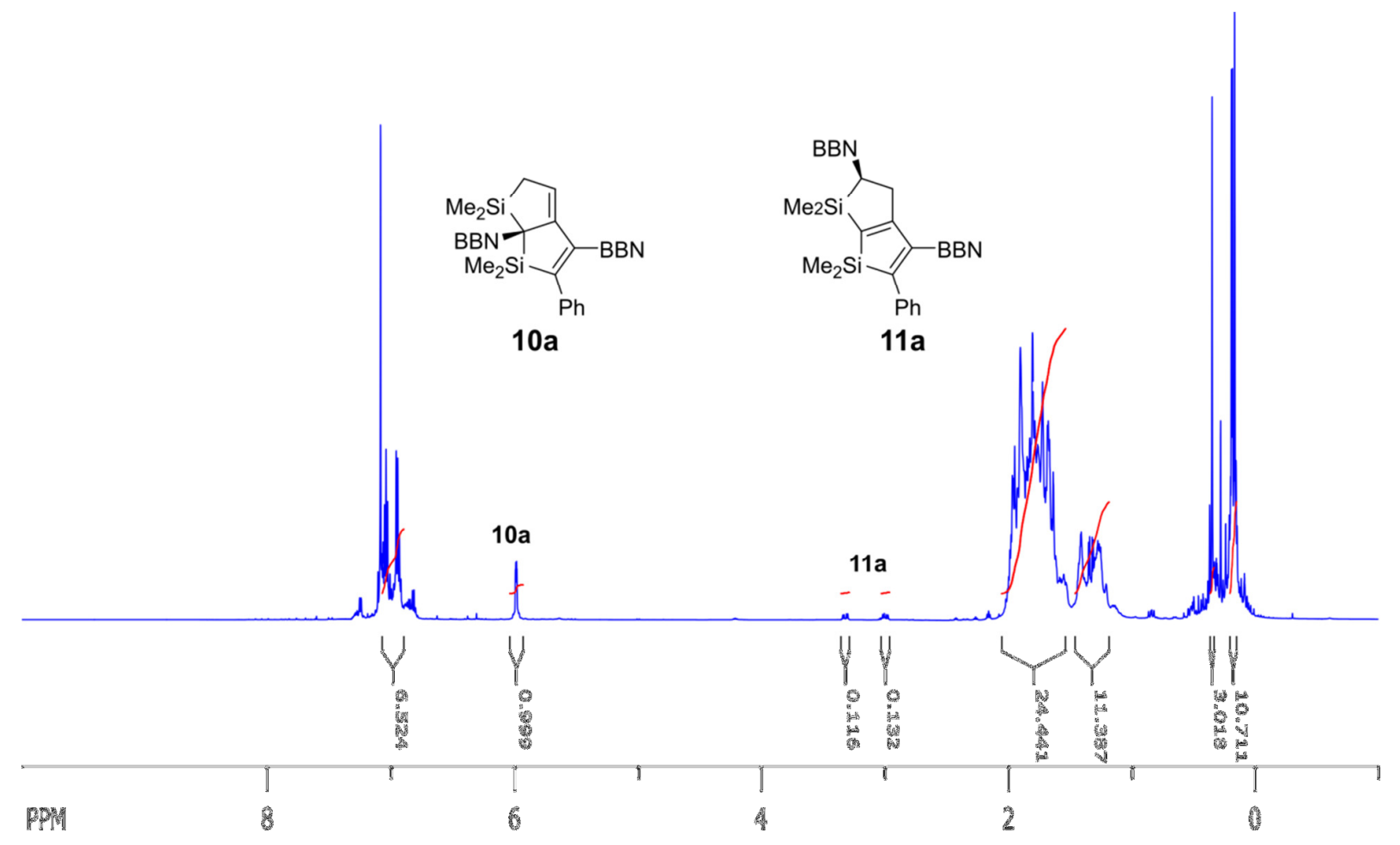

Figure S28. ${ }^{1} \mathrm{H}$ NMR spectrum $\left(600 \mathrm{MHz}, \mathrm{C}_{6} \mathrm{D}_{6}\right)$ of the reaction mixture containing 10a and 11a. 


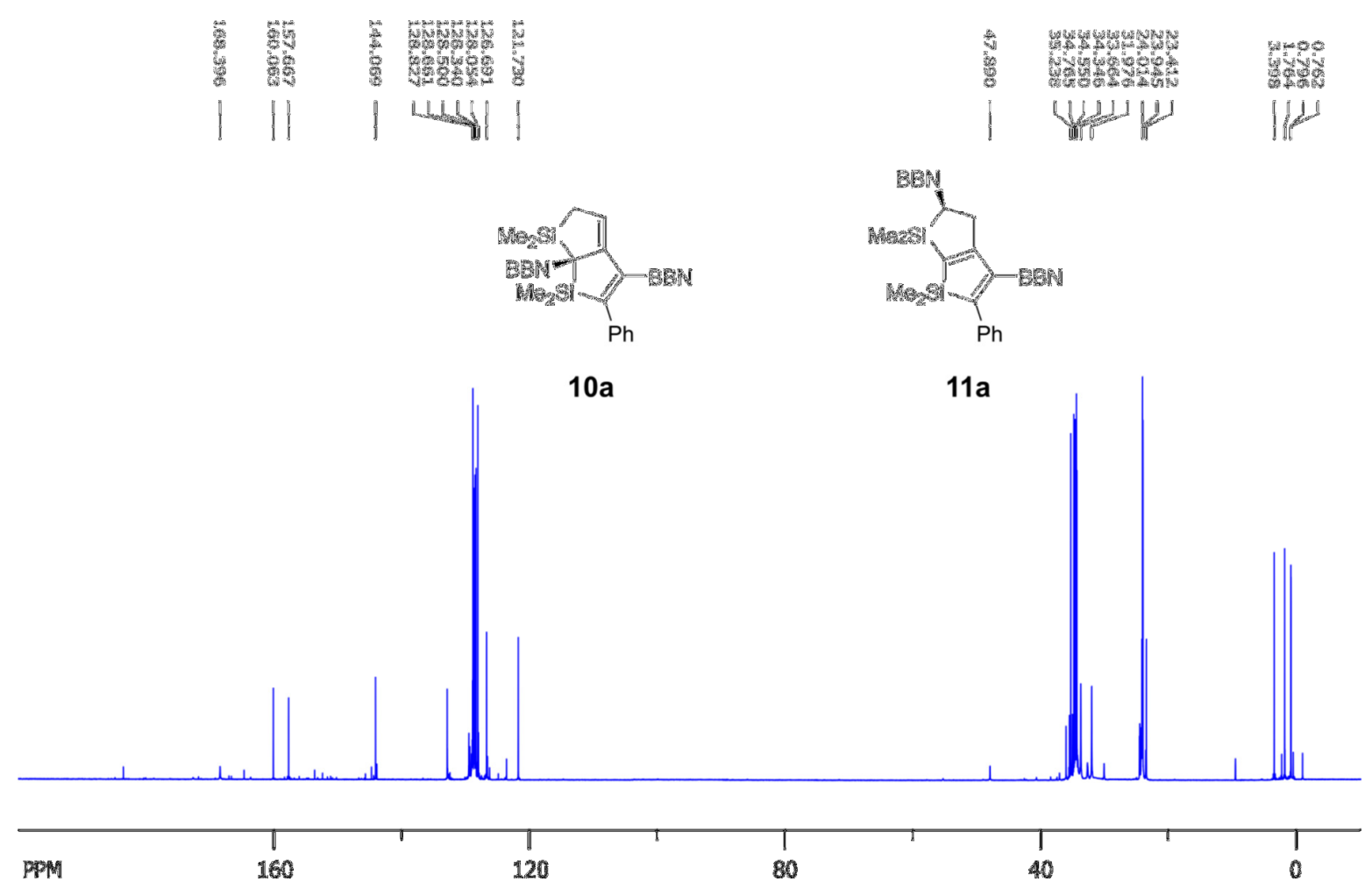

Figure S29. ${ }^{13} \mathrm{C}\left\{{ }^{1} \mathrm{H}\right\}$ NMR spectrum $\left(150.9 \mathrm{MHz}, \mathrm{C}_{6} \mathrm{D}_{6}\right)$ of the reaction mixture containing 10a and 11a.
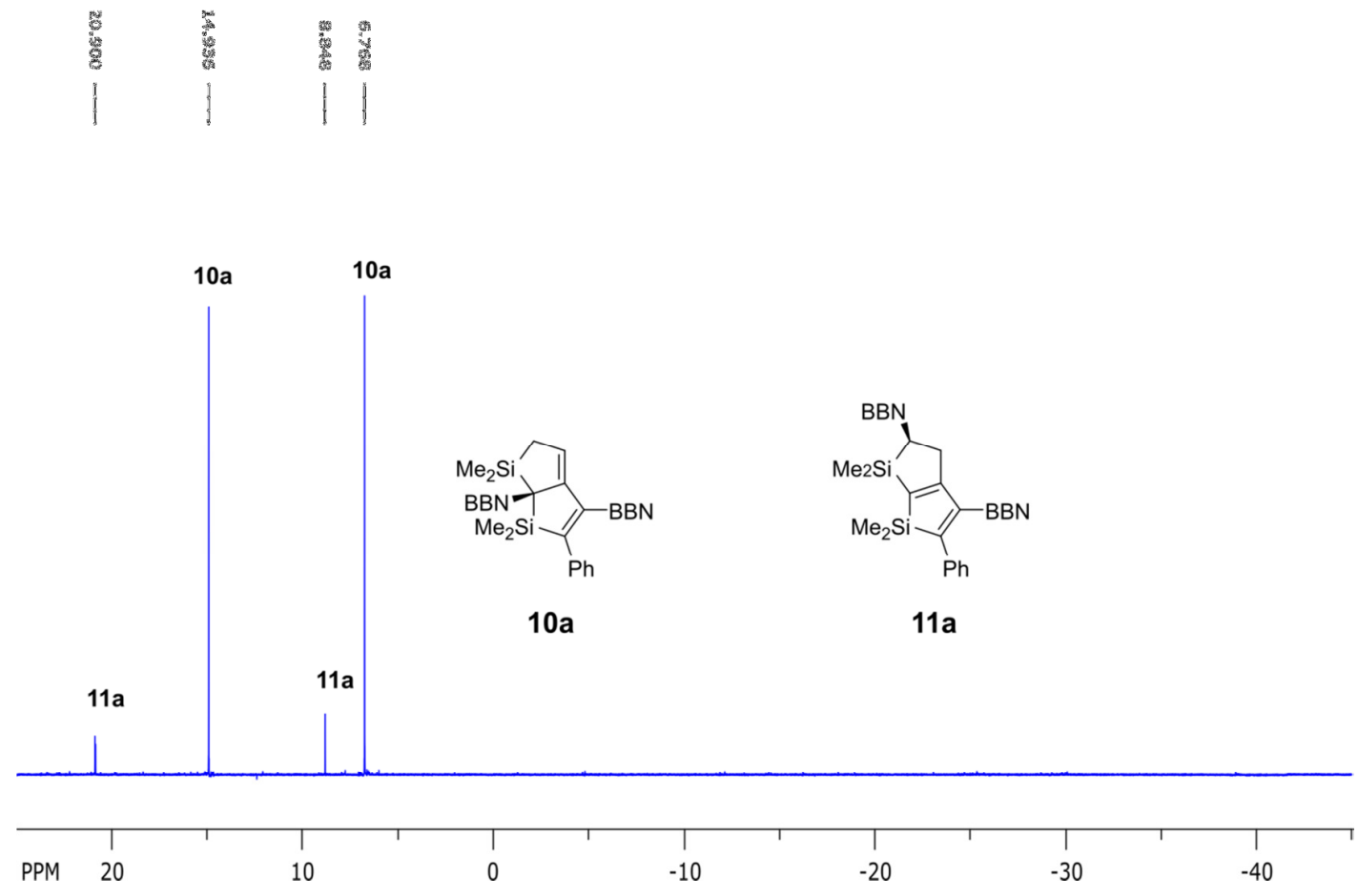

Figure S30. ${ }^{29} \mathrm{Si}\left\{{ }^{1} \mathrm{H}\right\}$ NMR spectrum (refocused INEPT, $119.2 \mathrm{MHz}, \mathrm{C}_{6} \mathrm{D}_{6}$ ) of the reaction mixture containing 10a and 11a. 


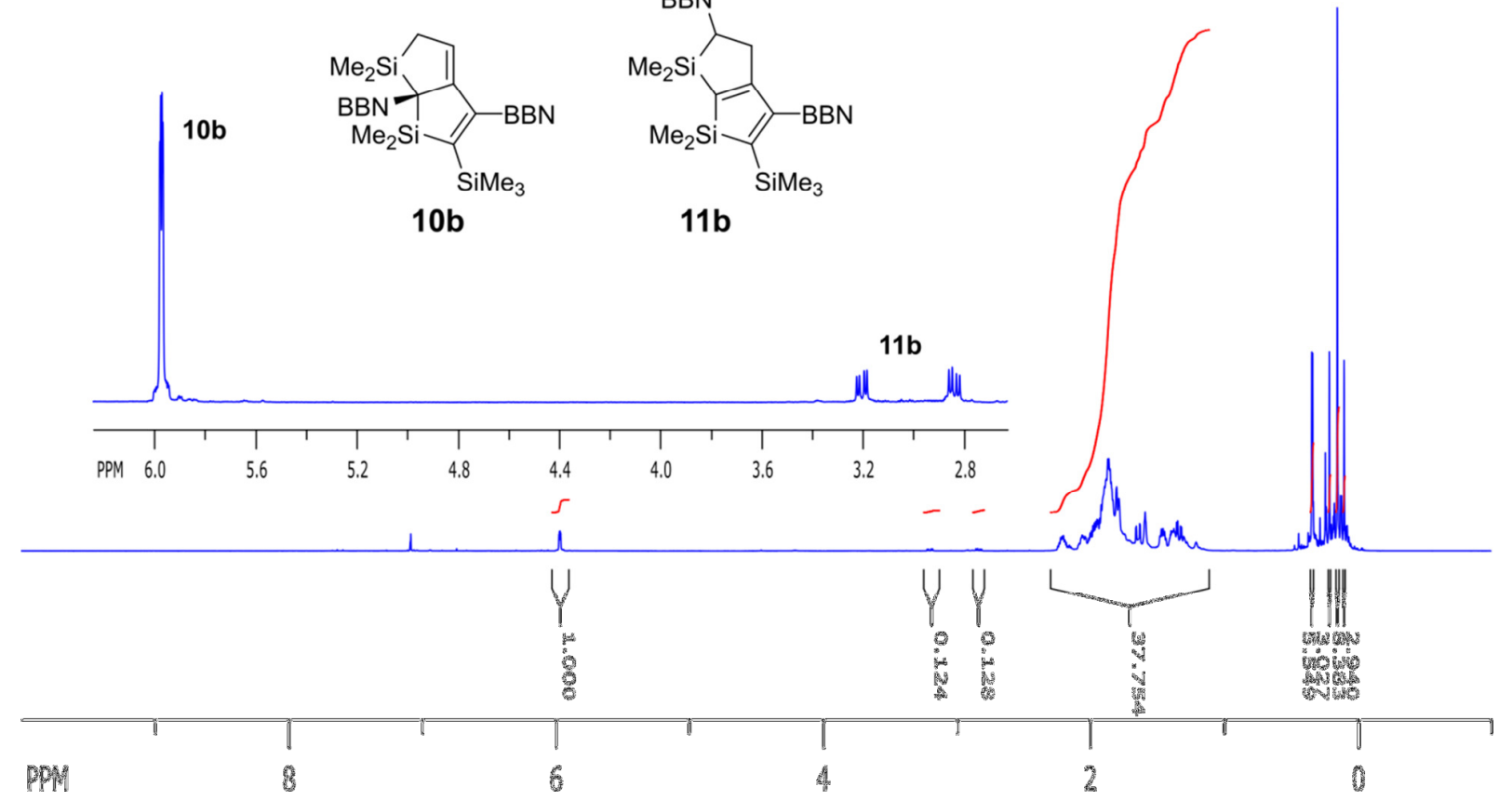

Figure S31. ${ }^{1} \mathrm{H}$ NMR spectrum $\left(600 \mathrm{MHz}, \mathrm{C}_{6} \mathrm{D}_{6}\right)$ of the reaction mixture containing $\mathbf{1 0 b}$ and $\mathbf{1 1 b}$.

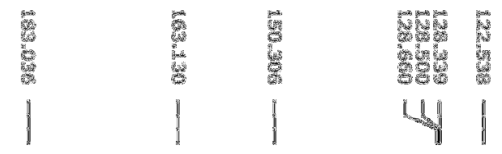
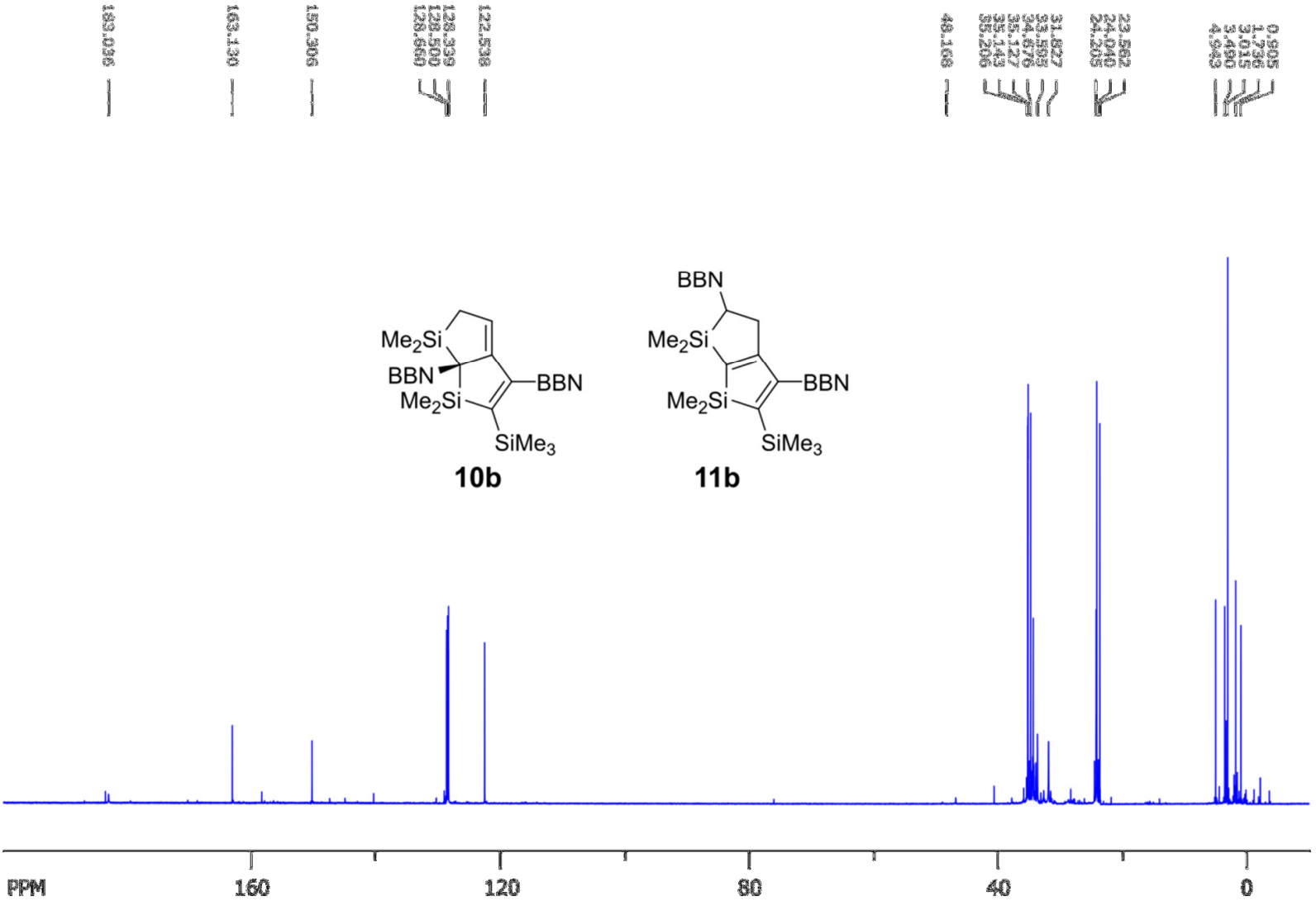

Figure S32. ${ }^{13} \mathrm{C}\left\{{ }^{1} \mathrm{H}\right\}$ NMR spectrum $\left(150.9 \mathrm{MHz}, \mathrm{C}_{6} \mathrm{D}_{6}\right)$ of the reaction mixture containing $\mathbf{1 0 b}$ and $\mathbf{1 1 b}$. 


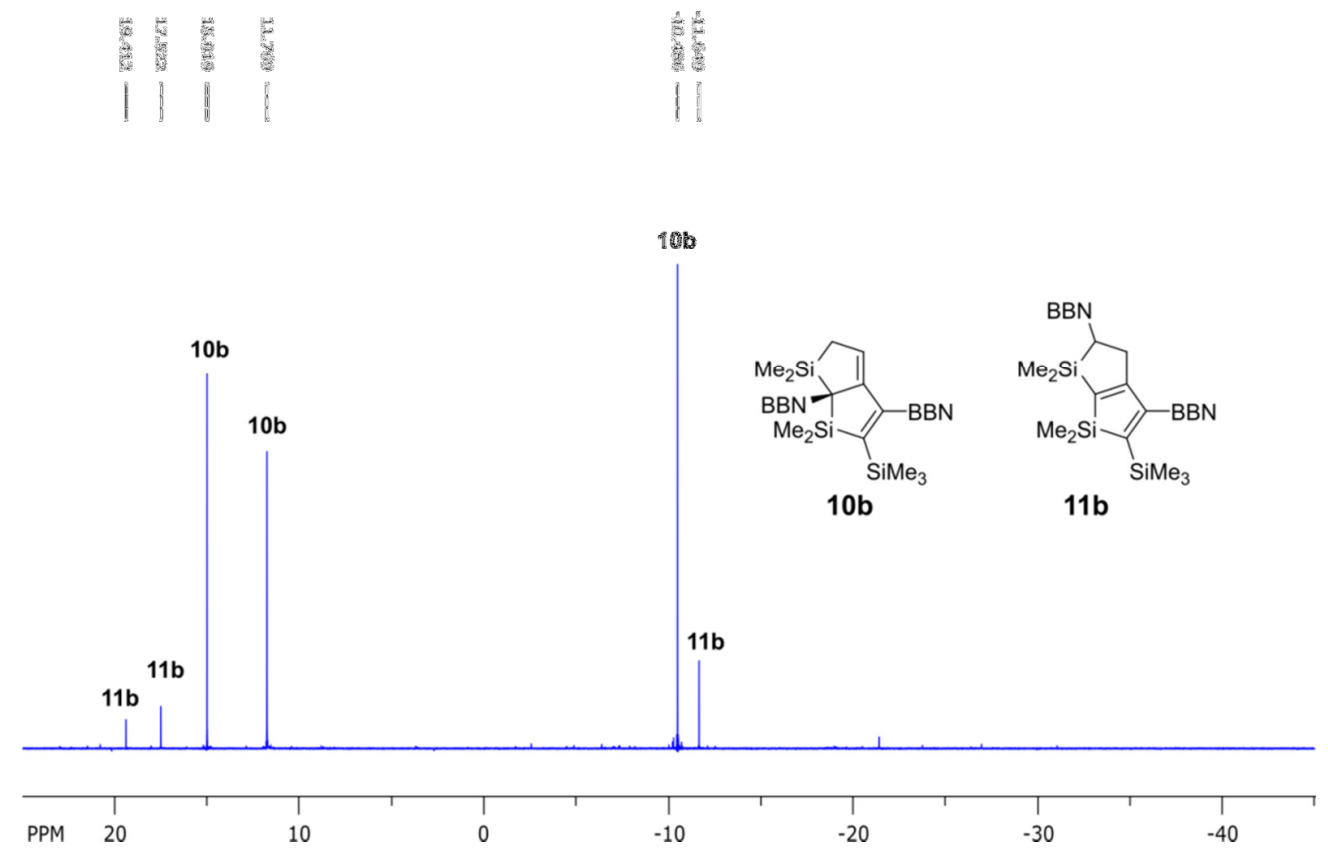

Figure S33. ${ }^{29} \mathrm{Si}\left\{{ }^{1} \mathrm{H}\right\}$ NMR spectrum (refocused INEPT, $119.2 \mathrm{MHz}, \mathrm{C}_{6} \mathrm{D}_{6}$ ) of the reaction mixture containing $\mathbf{1 0 b}$ and $\mathbf{1 1 b}$.

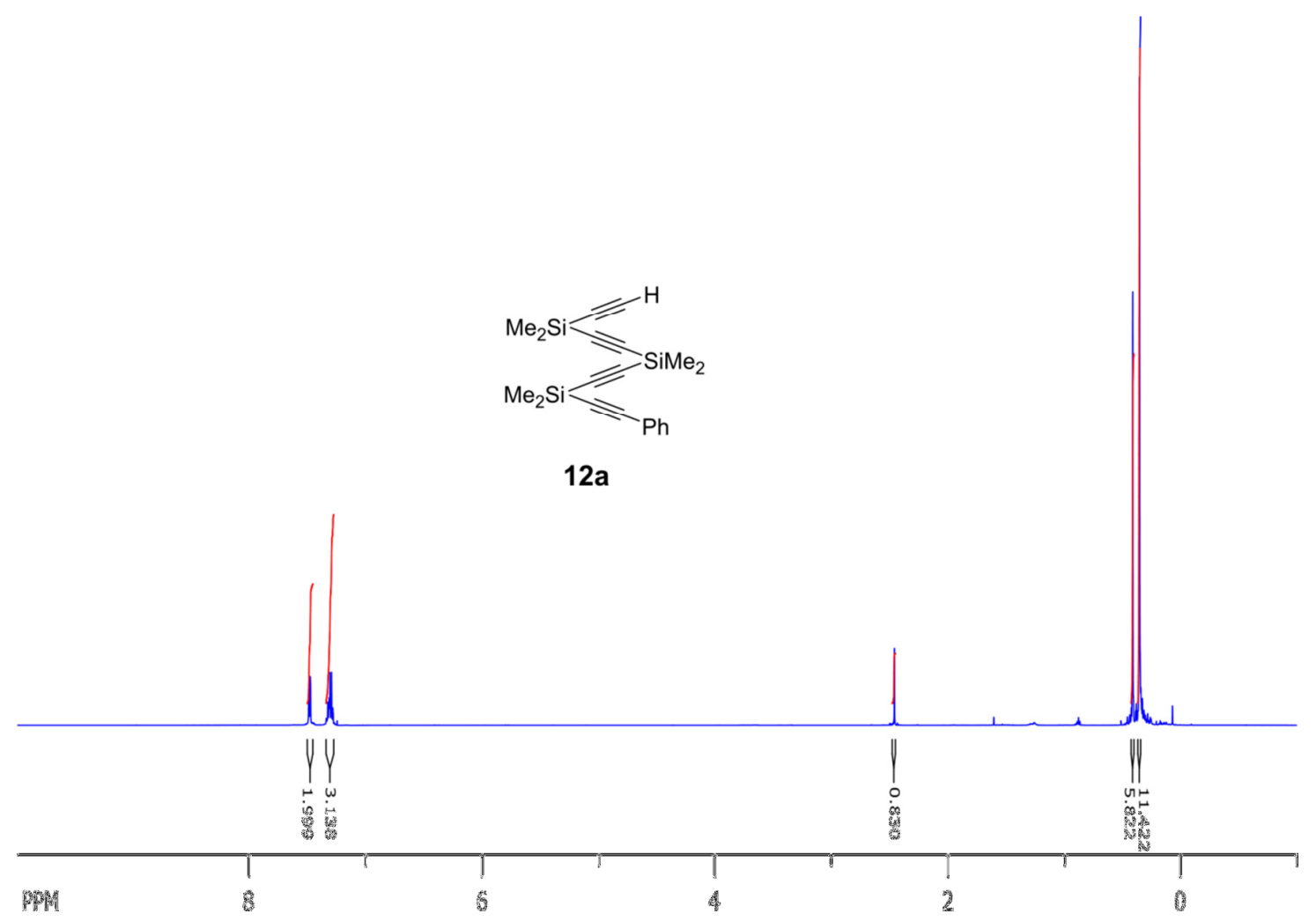

Figure S34. ${ }^{1} \mathrm{H}$ NMR spectrum $\left(600 \mathrm{MHz}, \mathrm{CDCl}_{3}\right)$ of 12a. 


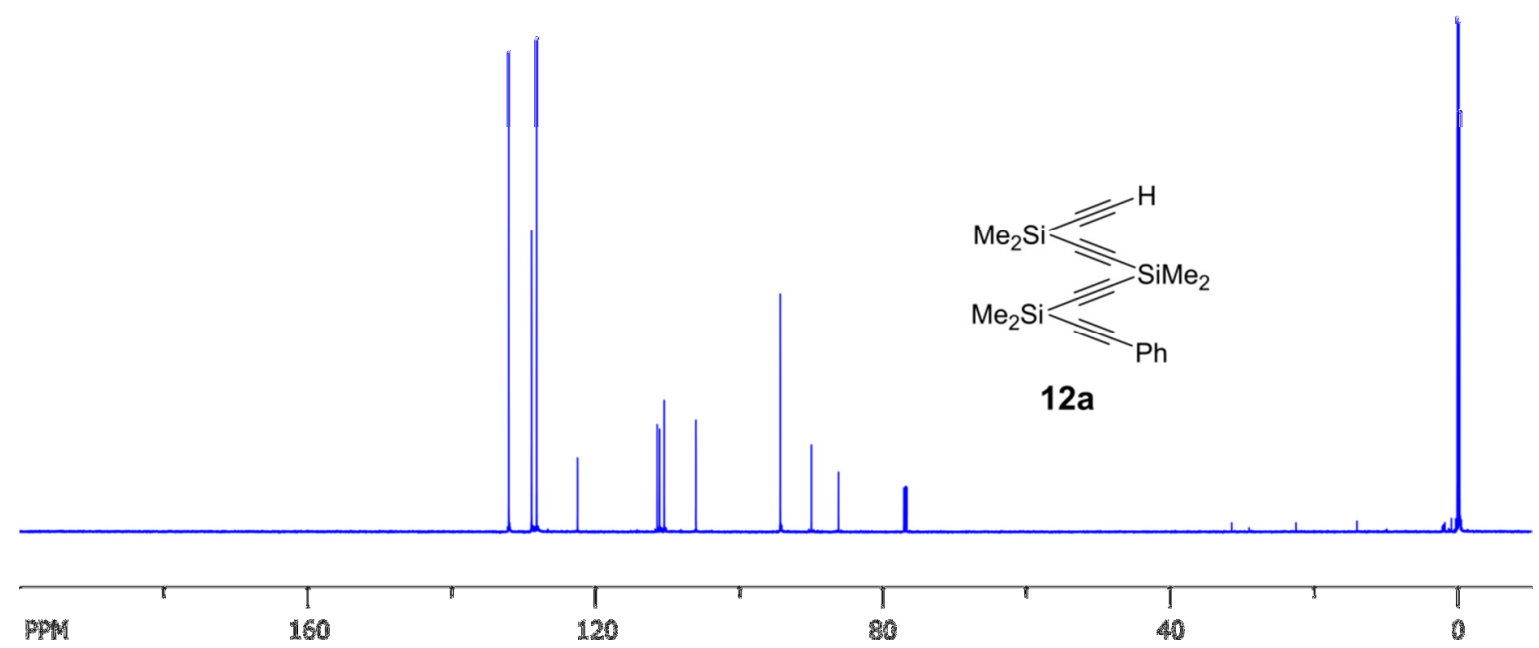

Figure S35. ${ }^{13} \mathrm{C}\left\{{ }^{1} \mathrm{H}\right\}$ NMR spectrum $\left(150.9 \mathrm{MHz}, \mathrm{CDCl}_{3}\right)$ of 12a.

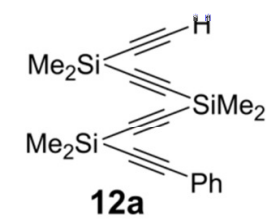

Figure S36. ${ }^{29} \mathrm{Si}\left\{{ }^{1} \mathrm{H}\right\}$ NMR spectrum (refocused INEPT, 119.2 $\mathrm{MHz}, \mathrm{CDCl}_{3}$ ) of 12a. 


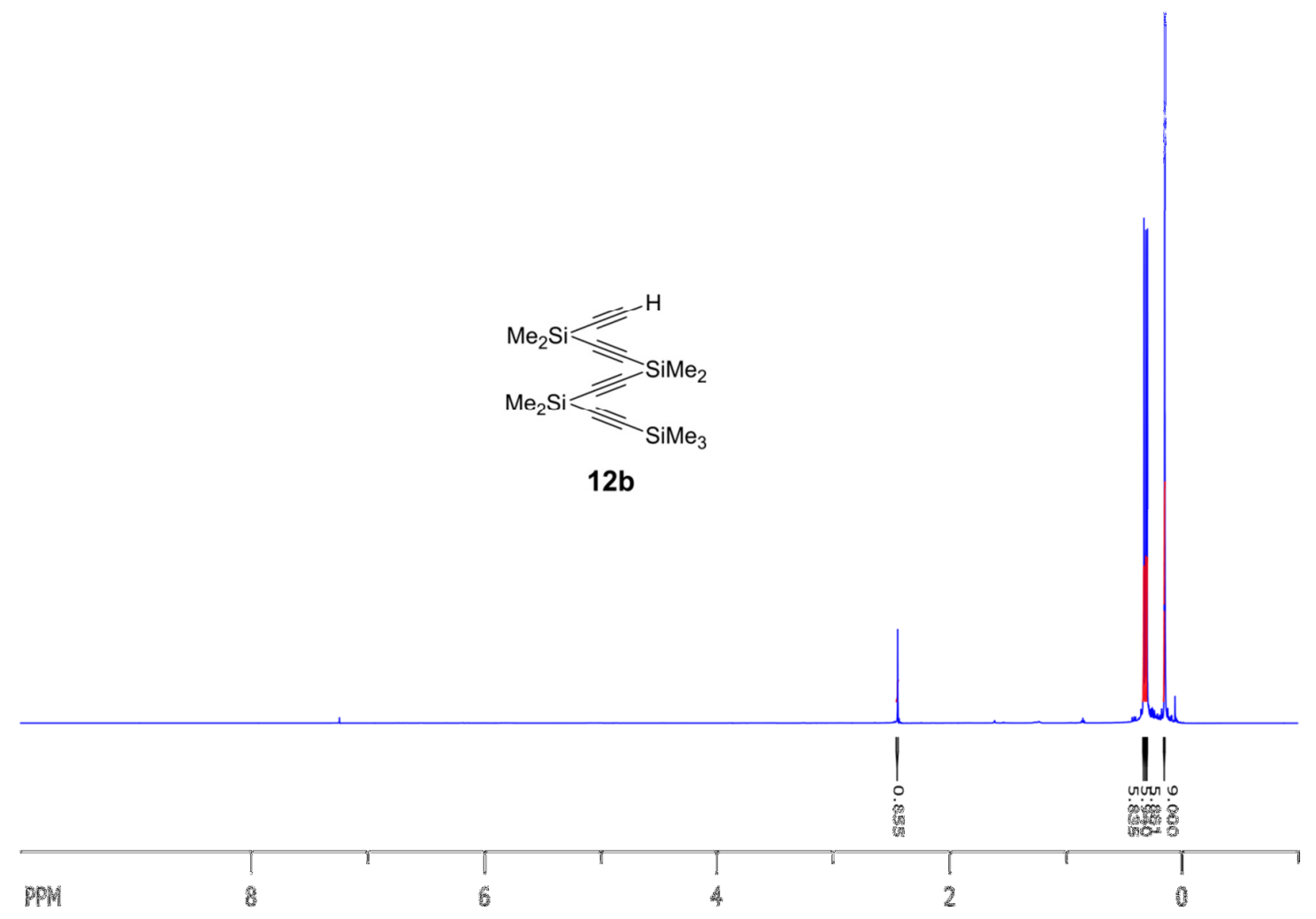

Figure S37. ${ }^{1} \mathrm{H}$ NMR spectrum $\left(600 \mathrm{MHz}, \mathrm{CDCl}_{3}\right)$ of $\mathbf{1 2 b}$.
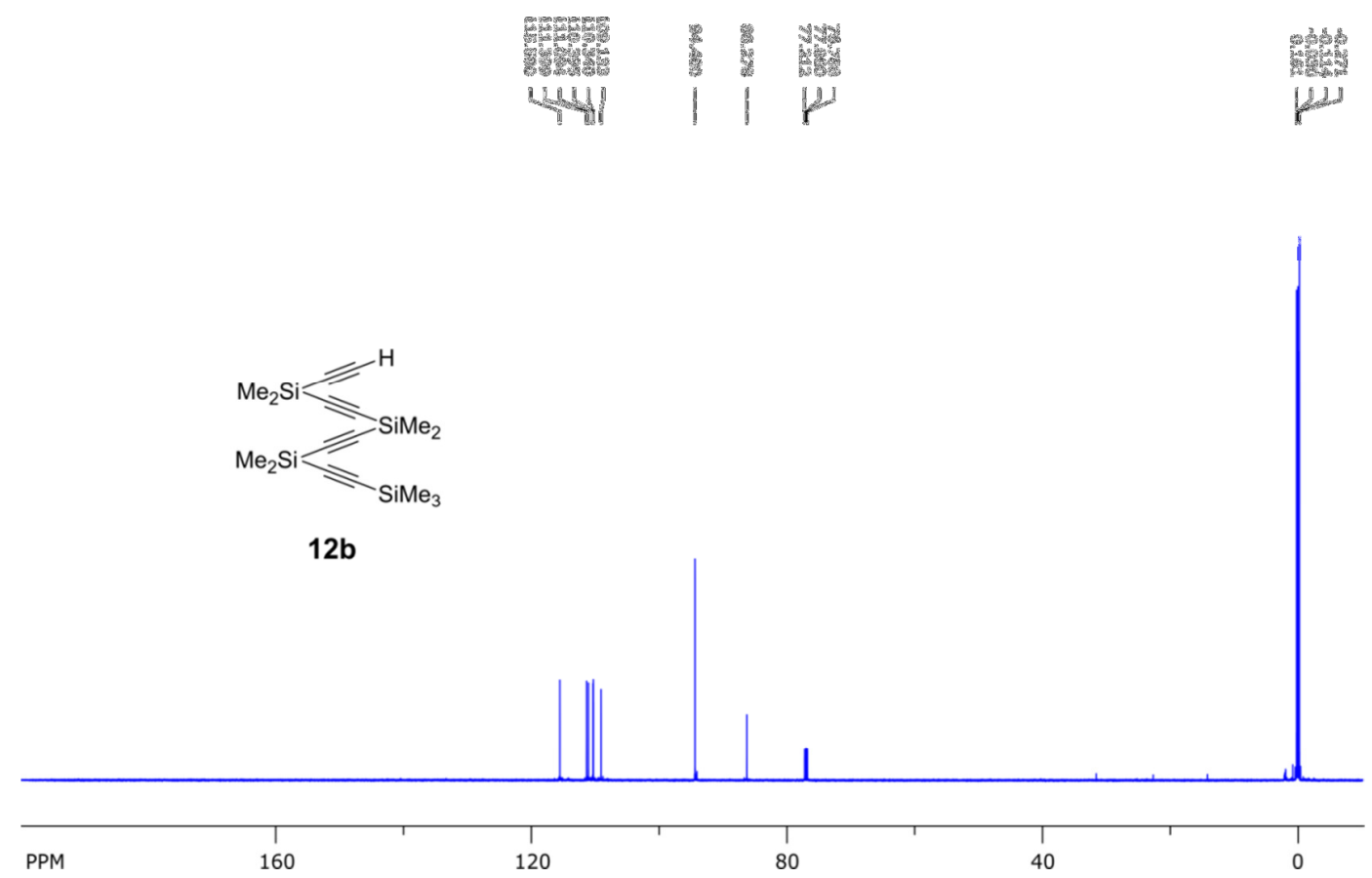

Figure S38. ${ }^{13} \mathrm{C}\left\{{ }^{1} \mathrm{H}\right\}$ NMR spectrum $\left(150.9 \mathrm{MHz}, \mathrm{CDCl}_{3}\right)$ of $\mathbf{1 2 b}$. 


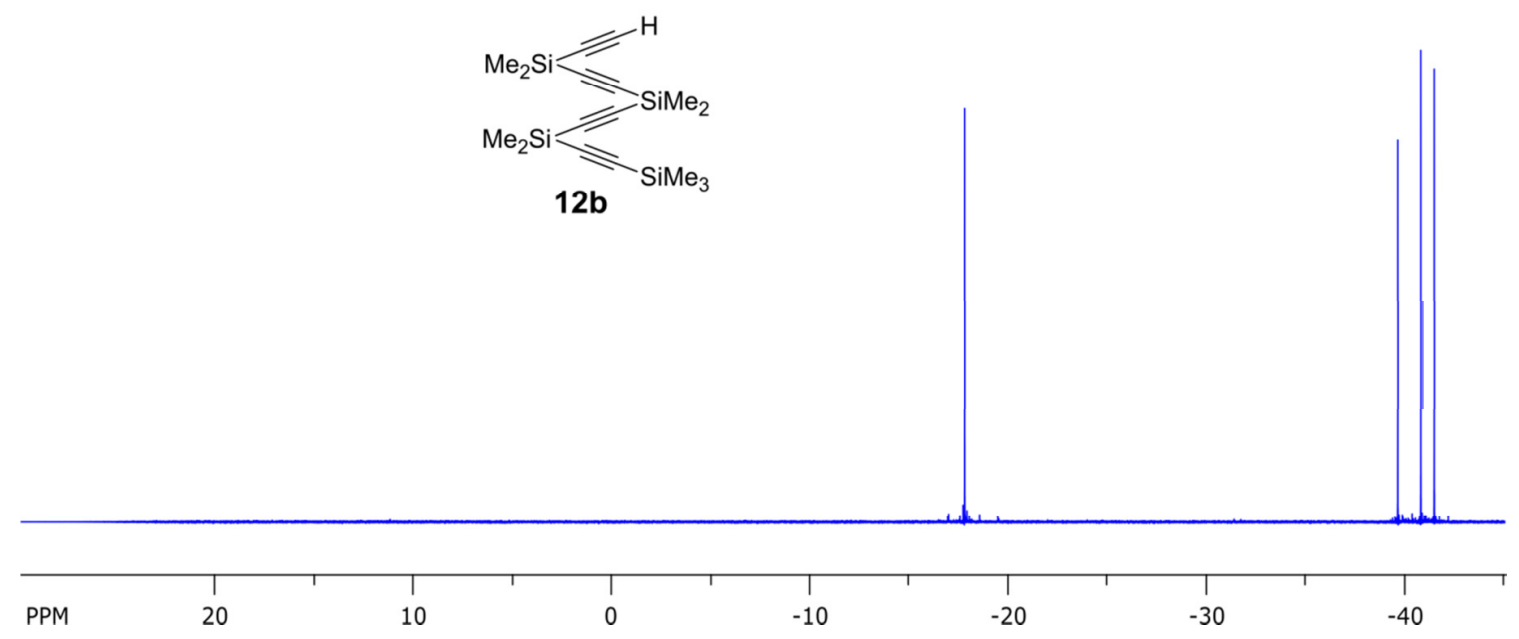

Figure S39. ${ }^{29} \mathrm{Si}\left\{{ }^{1} \mathrm{H}\right\}$ NMR spectrum (refocused INEPT, $119.2 \mathrm{MHz}, \mathrm{CDCl}_{3}$ ) of $\mathbf{1 2 b}$.

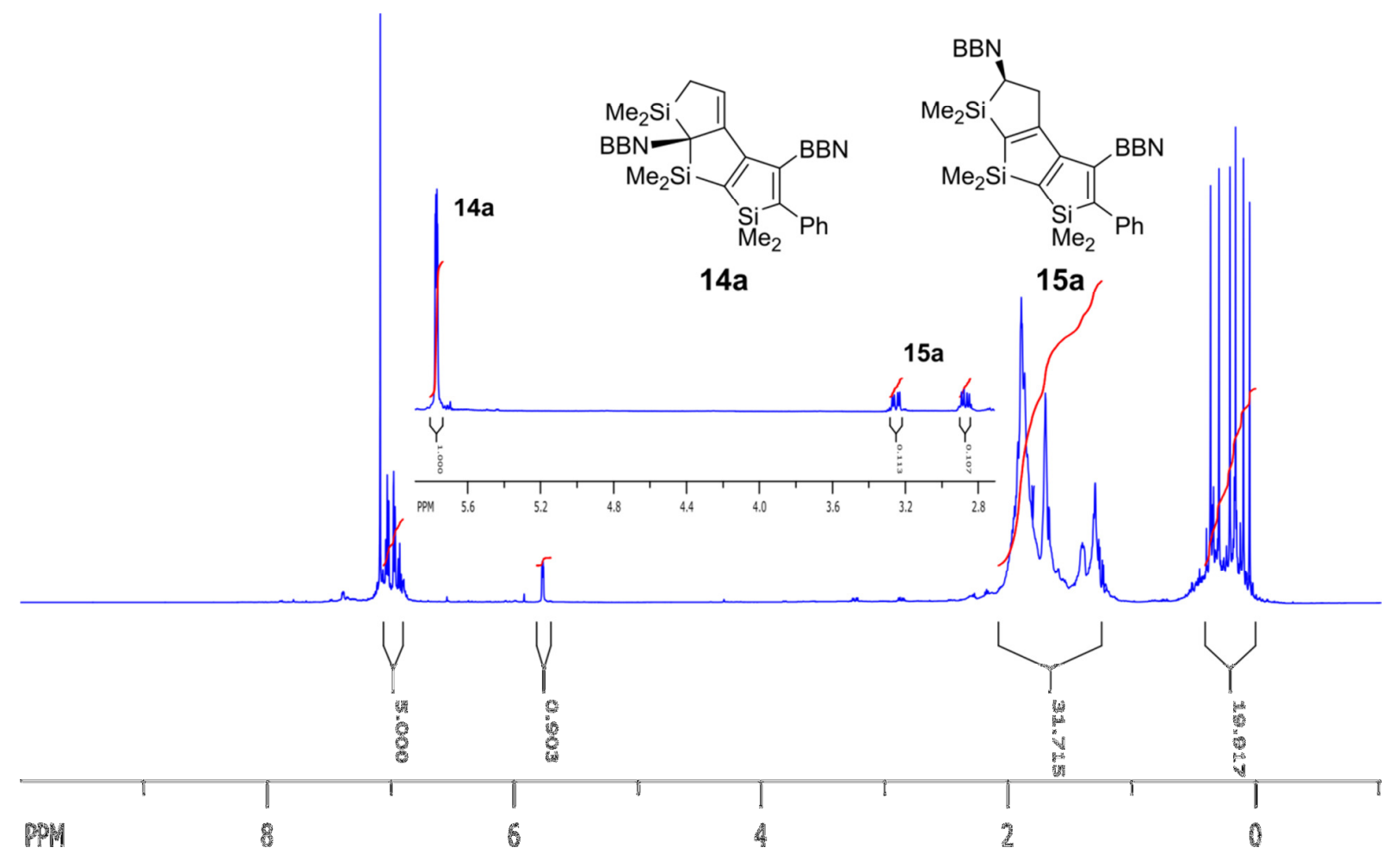

Figure S40. ${ }^{1} \mathrm{H}$ NMR spectrum $\left(600 \mathrm{MHz}, \mathrm{C}_{6} \mathrm{D}_{6}\right)$ of the reaction mixture containing $14 \mathbf{a}$ and $15 \mathrm{a}$. 


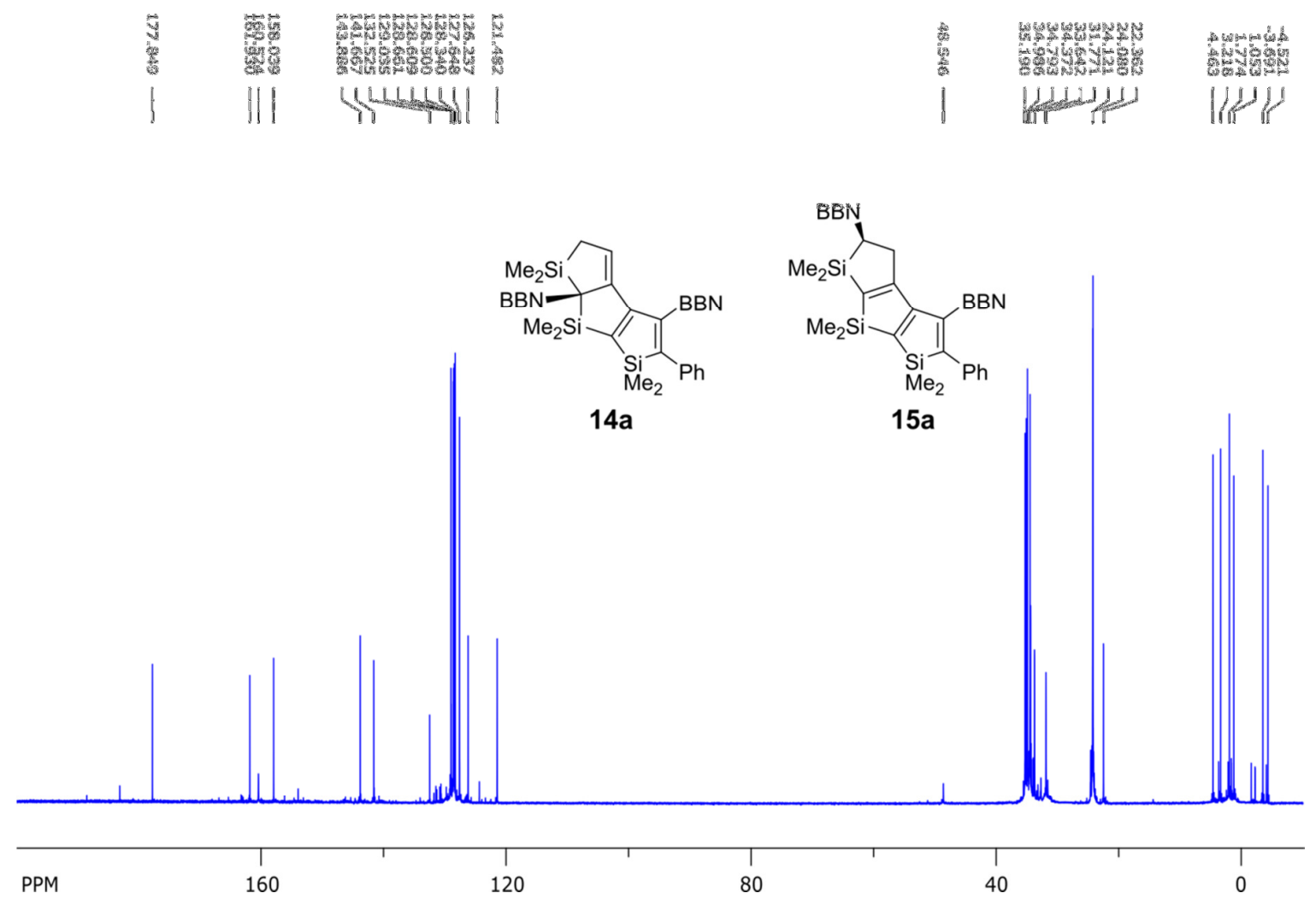

Figure S41. ${ }^{13} \mathrm{C}\left\{{ }^{1} \mathrm{H}\right\}$ NMR spectrum $\left(150.9 \mathrm{MHz}, \mathrm{C}_{6} \mathrm{D}_{6}\right)$ of the reaction mixture containing $\mathbf{1 4 a}$ and $\mathbf{1 5 a}$.
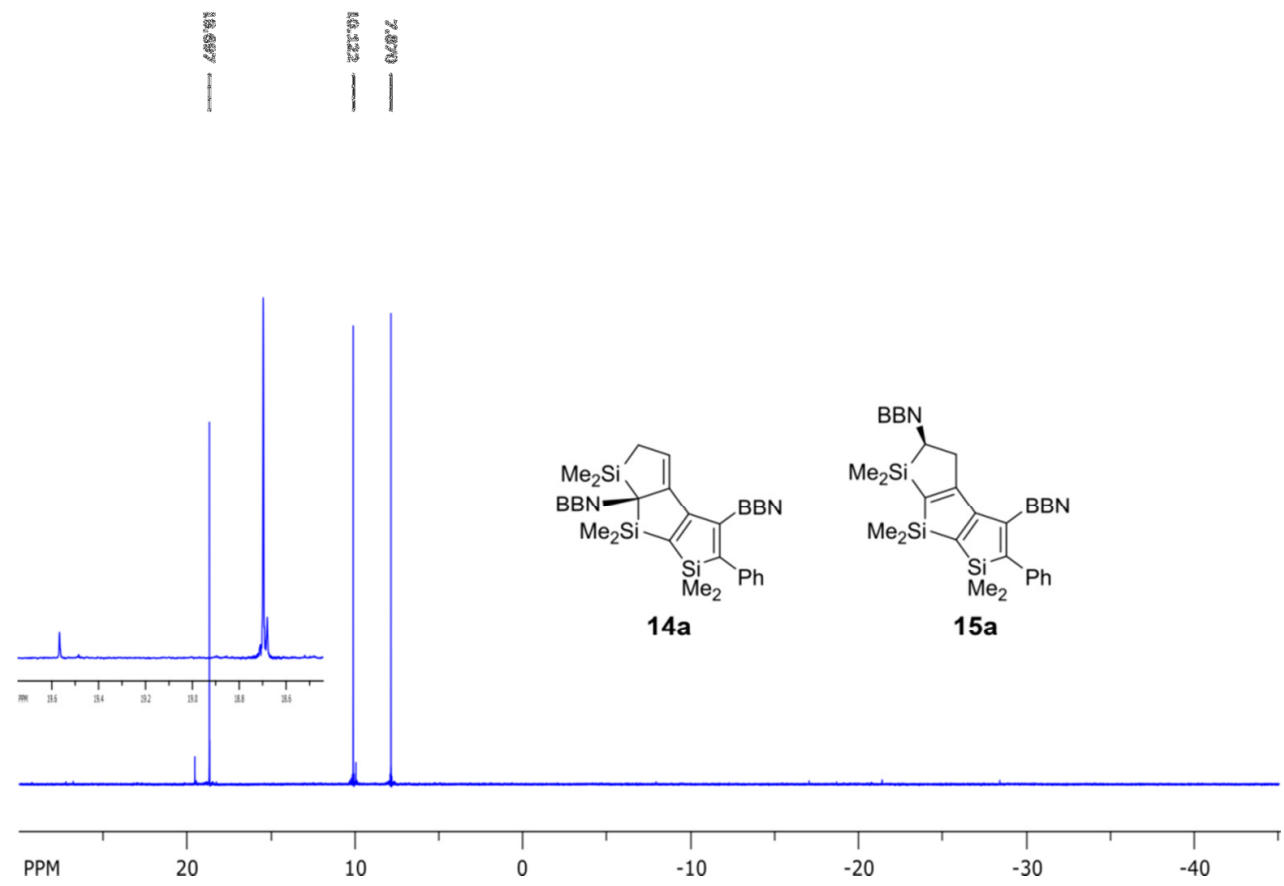

Figure $\mathbf{S 4 2} .{ }^{29} \mathrm{Si}\left\{{ }^{1} \mathrm{H}\right\}$ NMR spectrum (refocused INEPT, $119.2 \mathrm{MHz}, \mathrm{C}_{6} \mathrm{D}_{6}$ ) of the reaction mixture containing 14a and 15a. 


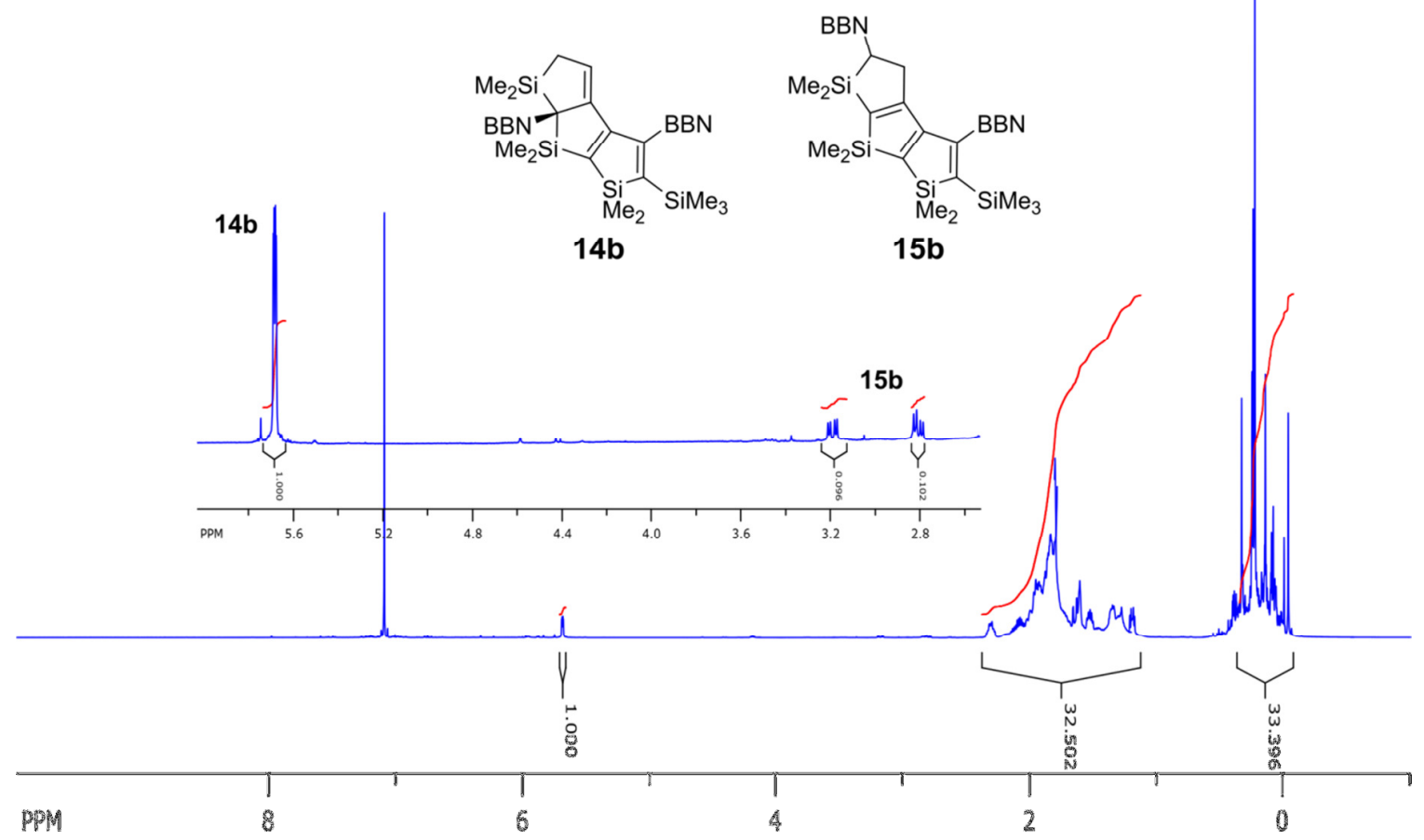

Figure S43. ${ }^{1} \mathrm{H}$ NMR spectrum $\left(600 \mathrm{MHz}, \mathrm{C}_{6} \mathrm{D}_{6}\right)$ of the reaction mixture containing $\mathbf{1 4 b}$ and $\mathbf{1 5 b}$.

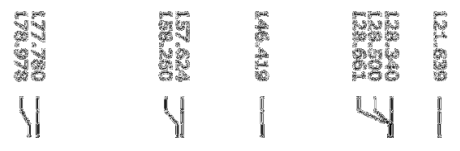

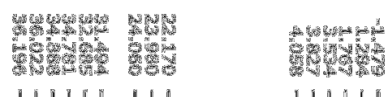

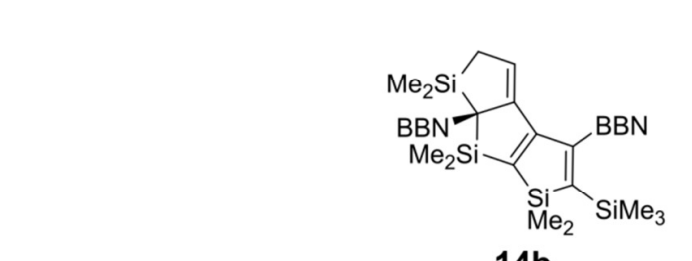

$14 \mathrm{~b}$

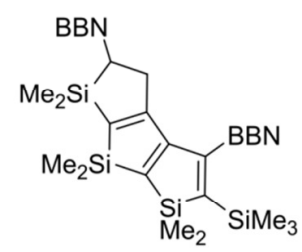

$15 b$

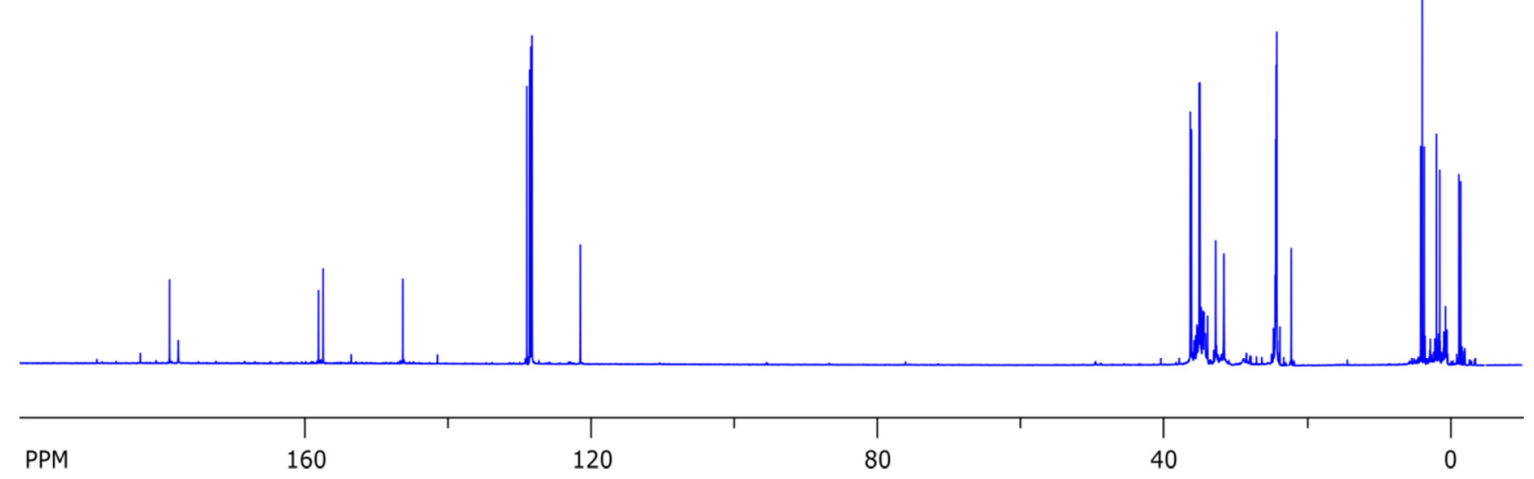

Figure S44. ${ }^{13} \mathrm{C}\left\{{ }^{1} \mathrm{H}\right\}$ NMR spectrum $\left(150.9 \mathrm{MHz}, \mathrm{C}_{6} \mathrm{D}_{6}\right)$ of the reaction mixture containing $\mathbf{1 4 b}$ and $\mathbf{1 5 b}$. 


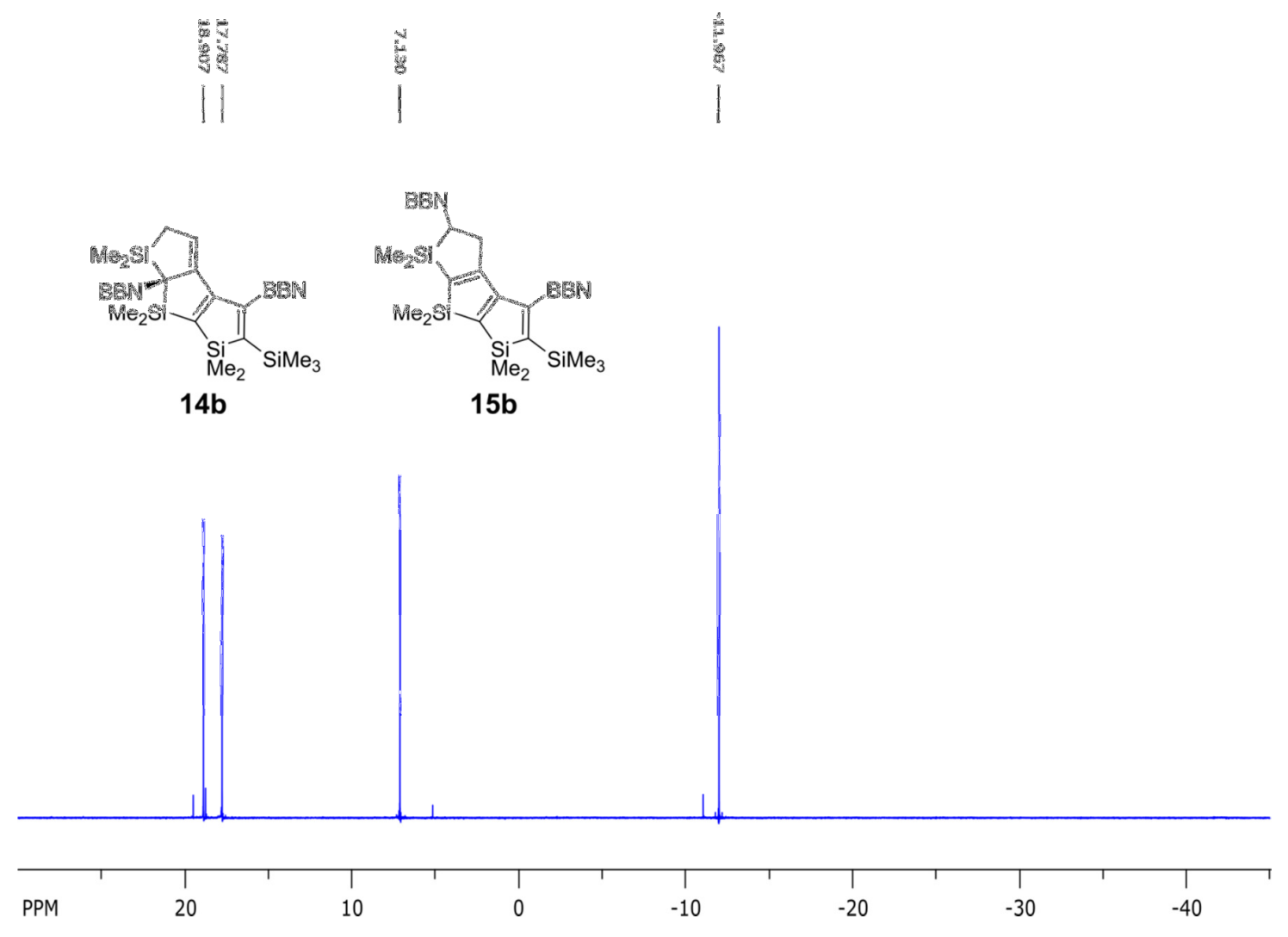

Figure S45. ${ }^{29} \mathrm{Si}\left\{{ }^{1} \mathrm{H}\right\}$ NMR spectrum (refocused INEPT, $119.2 \mathrm{MHz}, \mathrm{C}_{6} \mathrm{D}_{6}$ ) of the reaction mixture containing 14b and $\mathbf{1 5 b}$.

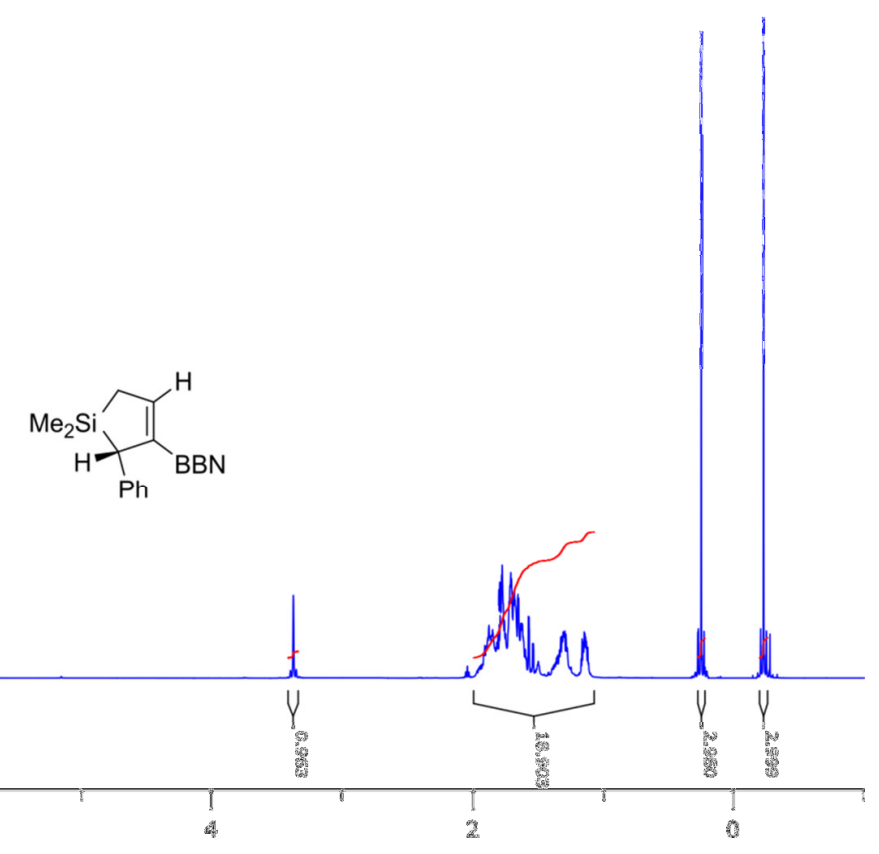

Figure S46. ${ }^{1} \mathrm{H}$ NMR spectrum $\left(600 \mathrm{MHz}, \mathrm{C}_{6} \mathrm{D}_{6}\right)$ of $\mathbf{1 6 a}$. 


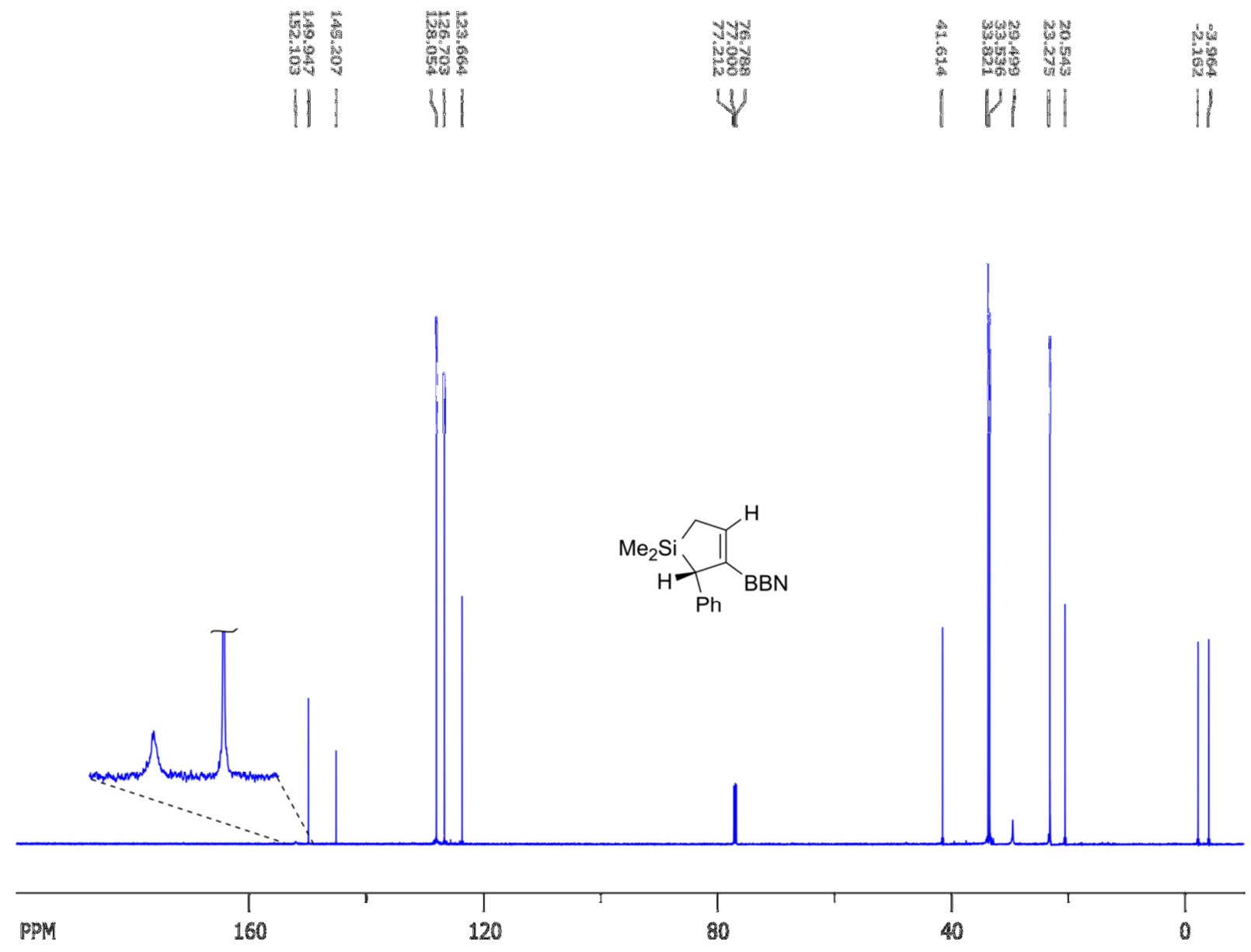

Figure S47. ${ }^{13} \mathrm{C}\left\{{ }^{1} \mathrm{H}\right\}$ NMR spectrum $\left(150.9 \mathrm{MHz}, \mathrm{CDCl}_{3}\right)$ of $\mathbf{1 6 a}$.

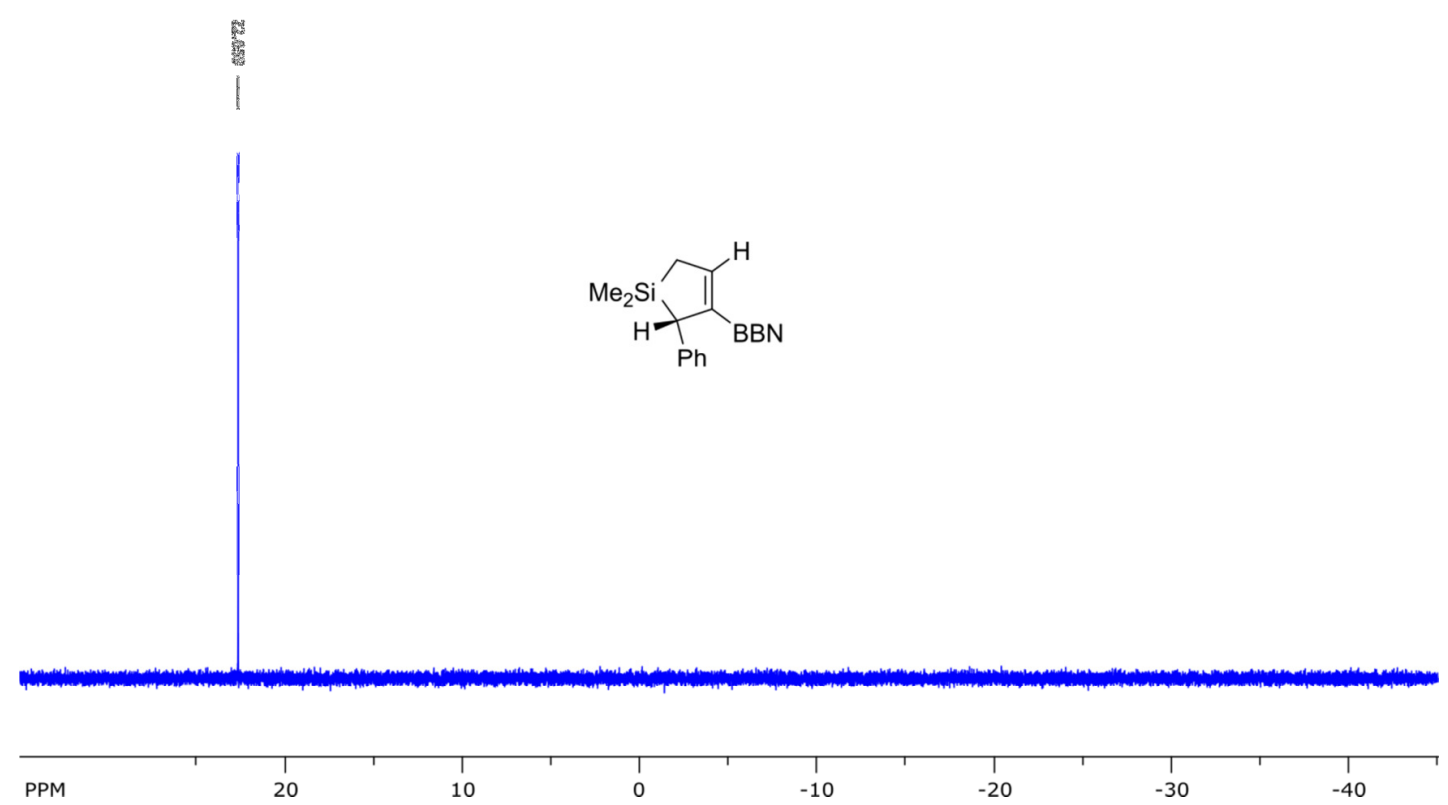

Figure S48. ${ }^{29} \mathrm{Si}\left\{{ }^{1} \mathrm{H}\right\}$ NMR spectrum (refocused INEPT, $119.2 \mathrm{MHz}, \mathrm{CDCl}_{3}$ ) of 16a. 


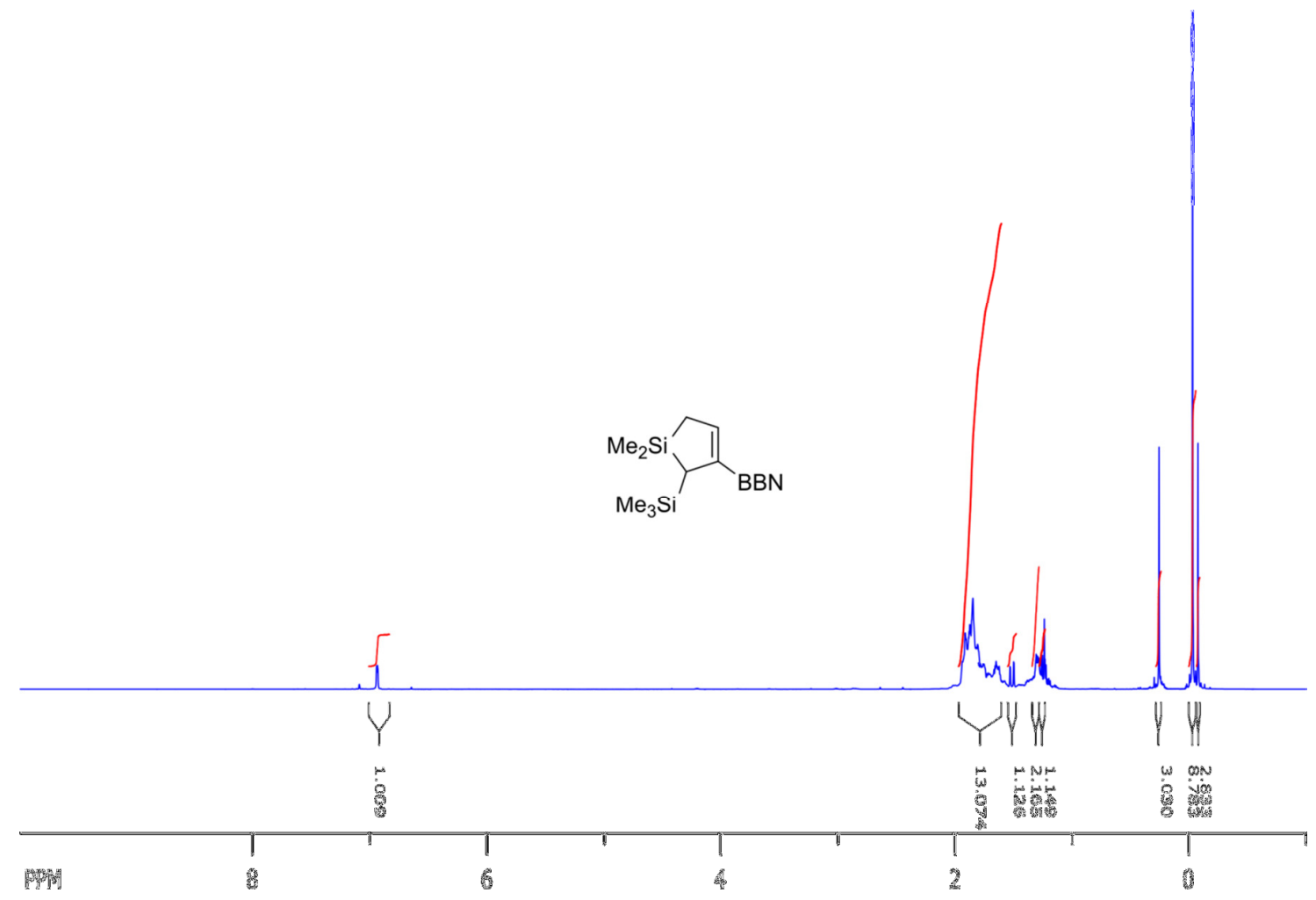

Figure S49. ${ }^{1} \mathrm{H}$ NMR spectrum $\left(600 \mathrm{MHz}, \mathrm{C}_{6} \mathrm{D}_{6}\right)$ of $\mathbf{1 6 b}$.

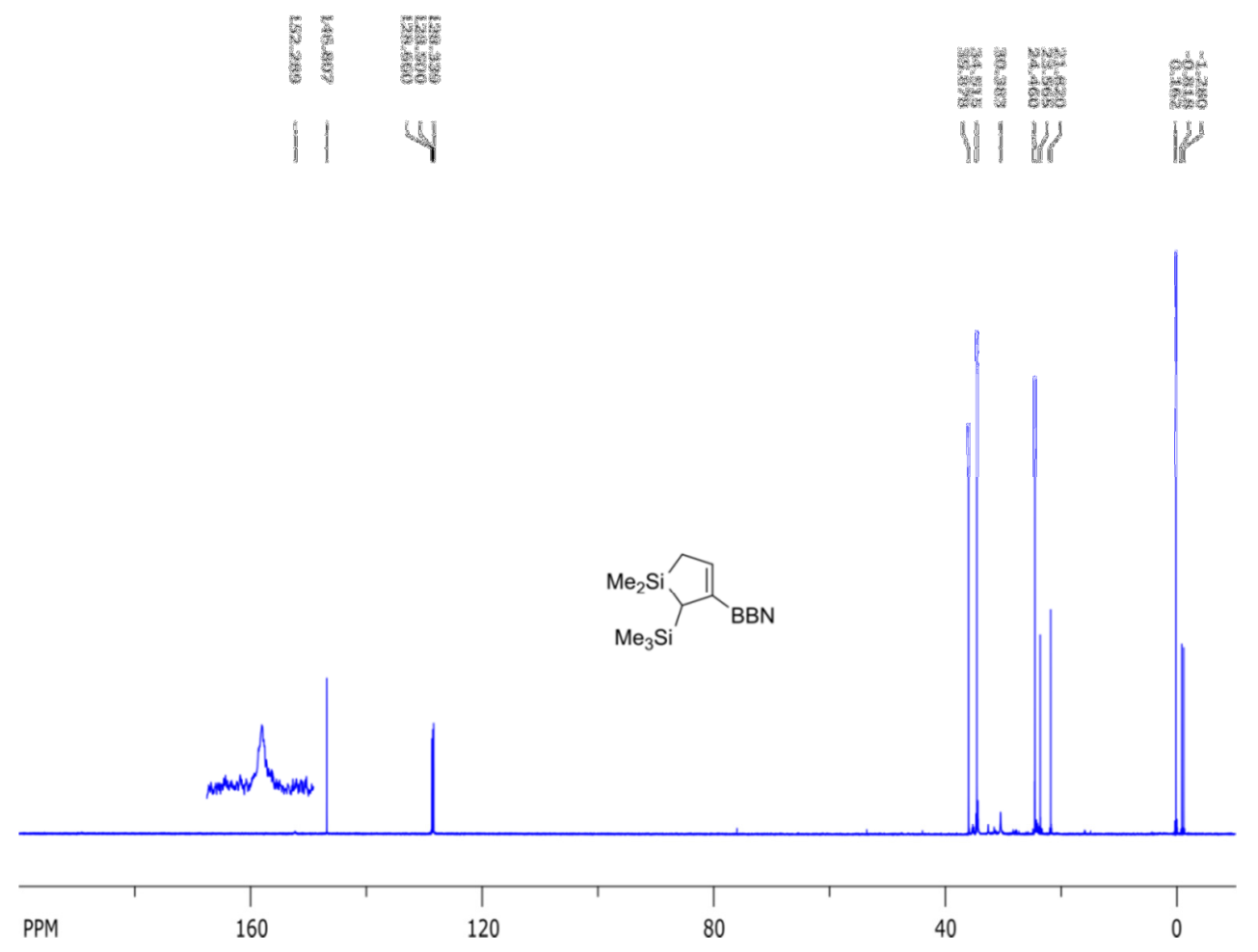

Figure S50. ${ }^{13} \mathrm{C}\left\{{ }^{1} \mathrm{H}\right\}$ NMR spectrum $\left(150.9 \mathrm{MHz}, \mathrm{C}_{6} \mathrm{D}_{6}\right)$ of $\mathbf{1 6 b}$. 

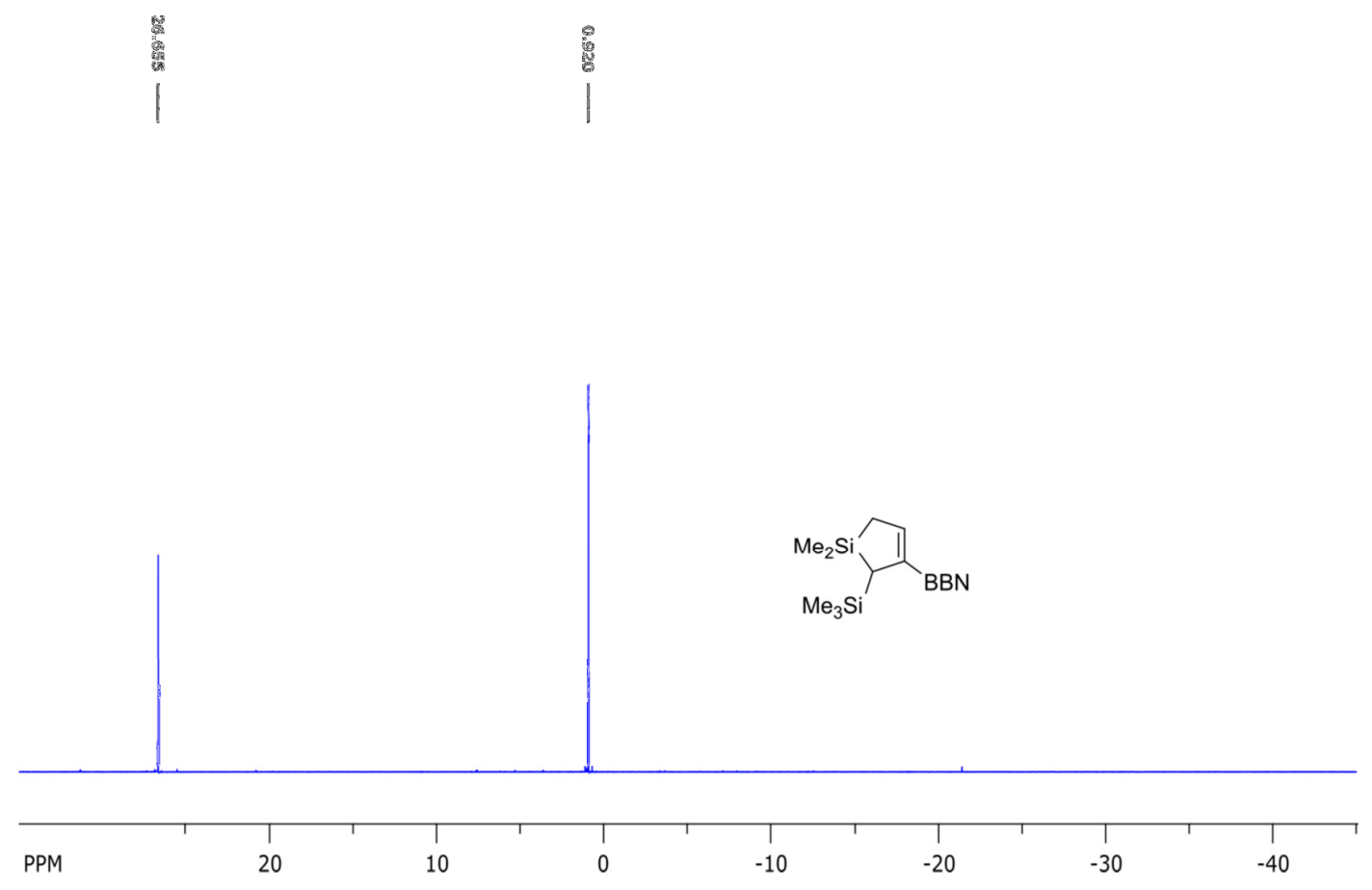

Figure S51. ${ }^{29} \mathrm{Si}\left\{{ }^{1} \mathrm{H}\right\}$ NMR spectrum (refocused INEPT, 119.2 MHz, $\mathrm{C}_{6} \mathrm{D}_{6}$ ) of $\mathbf{1 6 b}$.

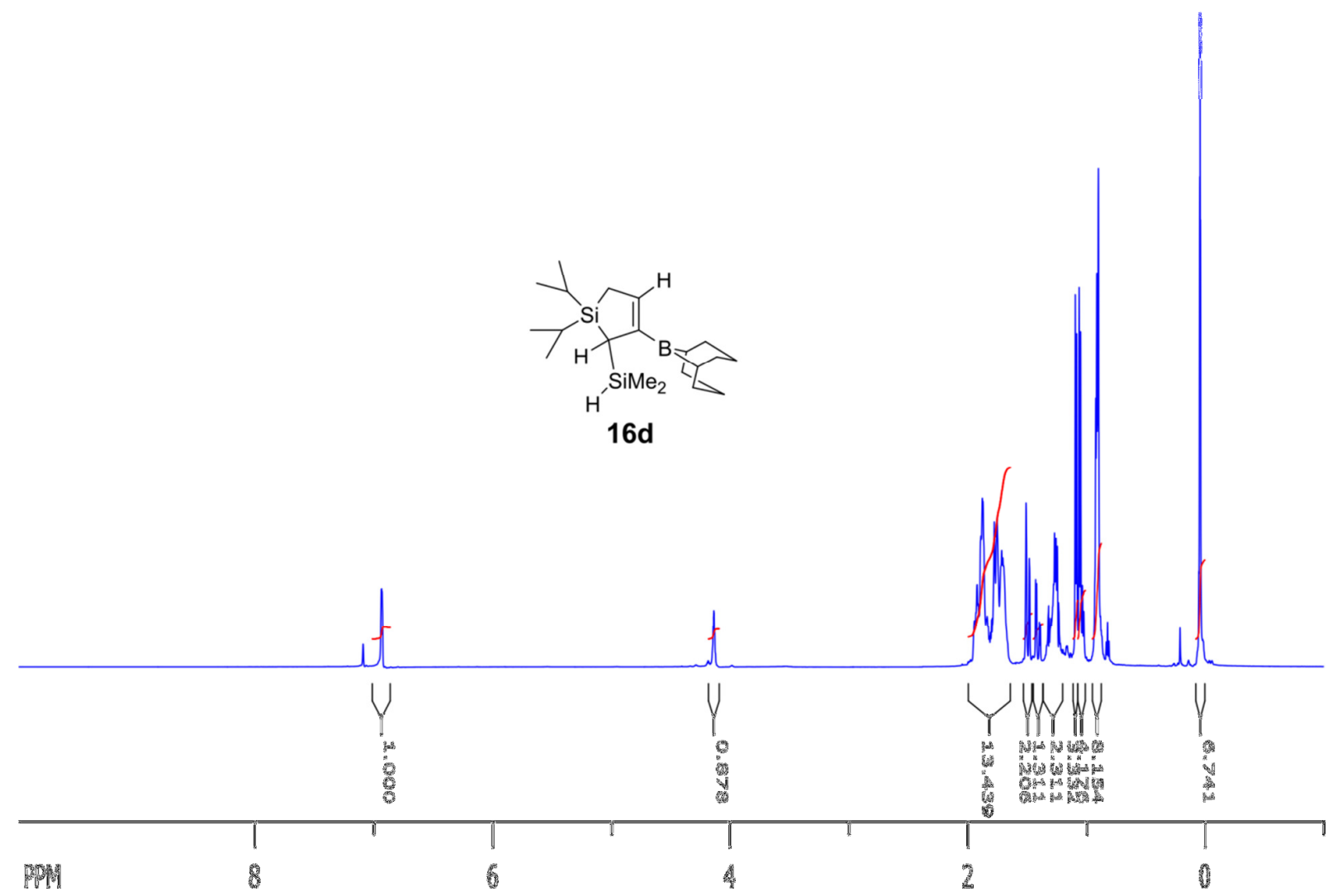

Figure S52. ${ }^{1} \mathrm{H}$ NMR spectrum $\left(600 \mathrm{MHz}, \mathrm{C}_{6} \mathrm{D}_{6}\right)$ of $\mathbf{1 6 d}$. 


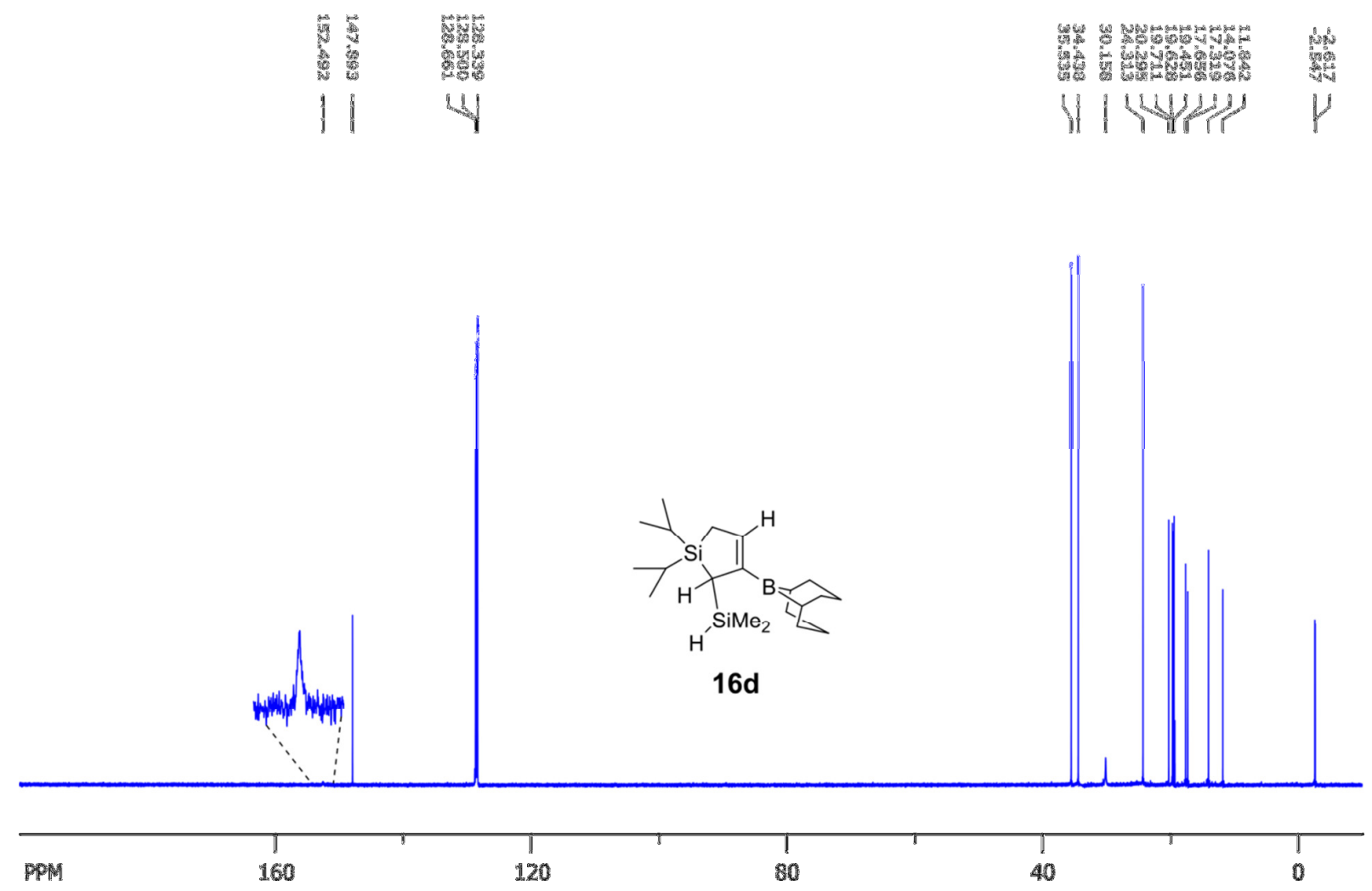

Figure S53. ${ }^{13} \mathrm{C}\left\{{ }^{1} \mathrm{H}\right\}$ NMR spectrum $\left(150.9 \mathrm{MHz}, \mathrm{C}_{6} \mathrm{D}_{6}\right)$ of $\mathbf{1 6 d}$.

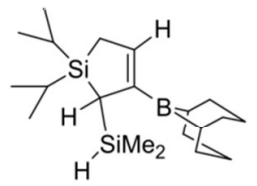

16d

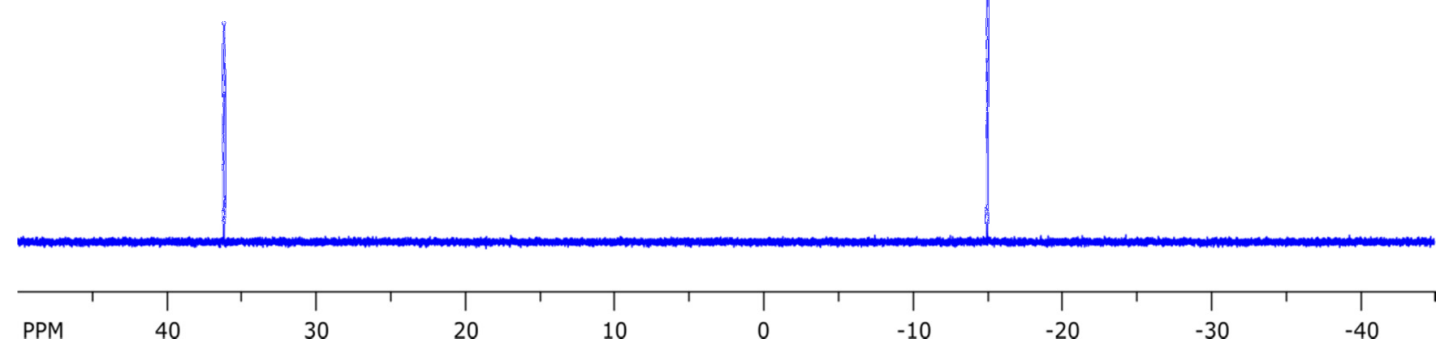

Figure S54. ${ }^{29} \mathrm{Si}\left\{{ }^{1} \mathrm{H}\right\}$ NMR spectrum (refocused INEPT, $119.2 \mathrm{MHz}, \mathrm{C}_{6} \mathrm{D}_{6}$ ) of $\mathbf{1 6 d}$. 


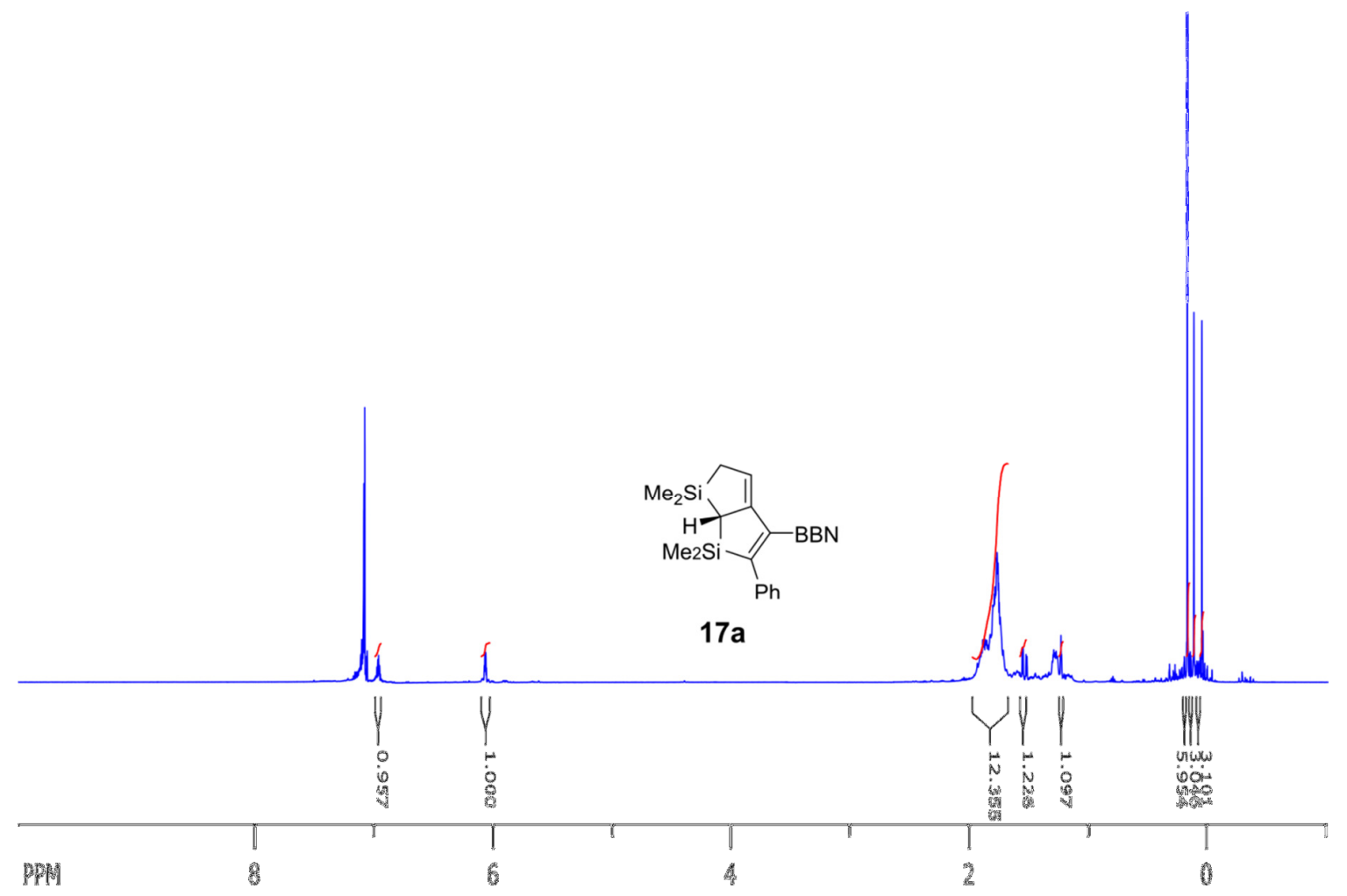

Figure S55. ${ }^{1} \mathrm{H}$ NMR spectrum $\left(600 \mathrm{MHz}, \mathrm{C}_{6} \mathrm{D}_{6}\right)$ of $\mathbf{1 7 a}$.

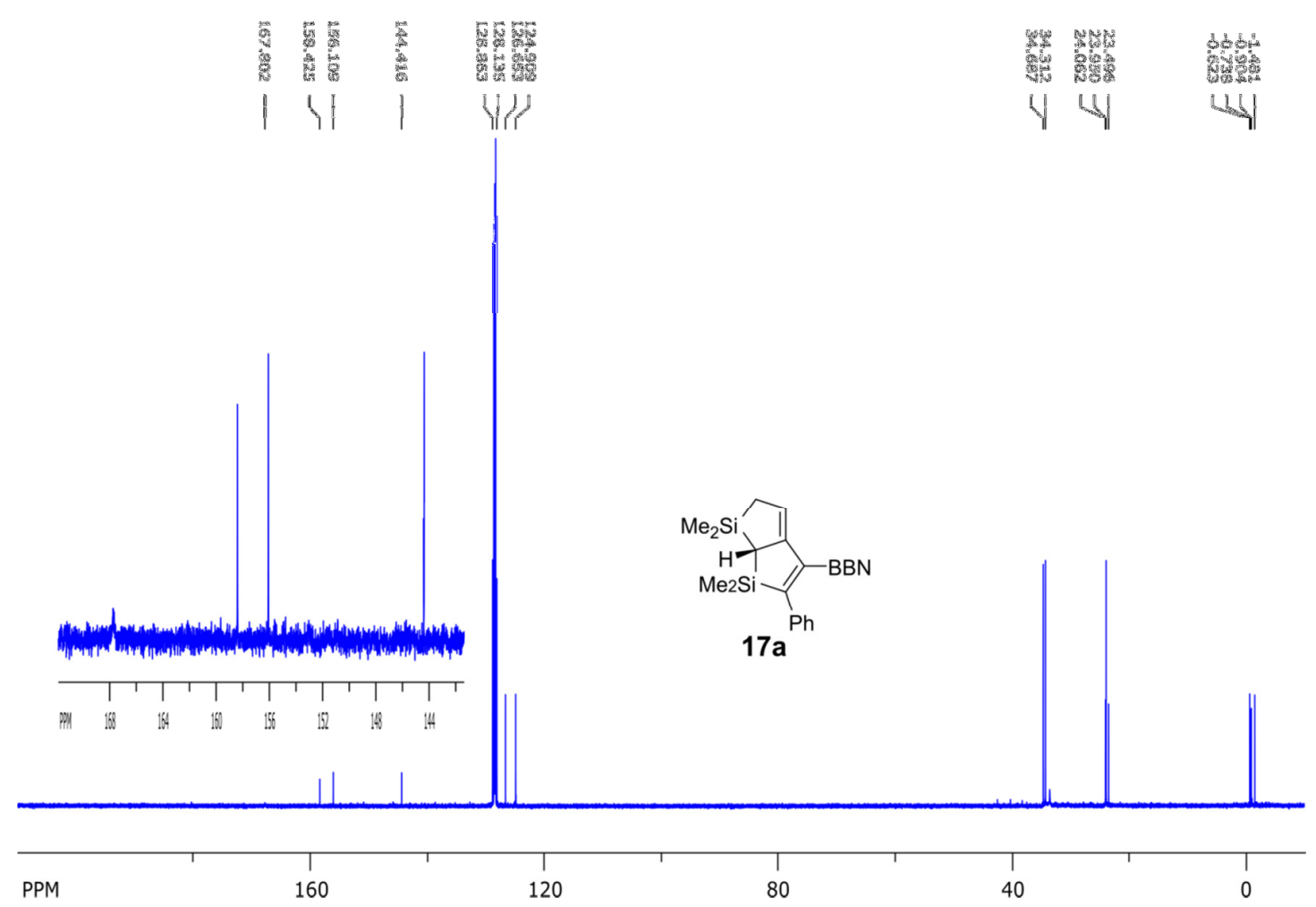

Figure S56. ${ }^{13} \mathrm{C}\left\{{ }^{1} \mathrm{H}\right\}$ NMR spectrum $\left(150.9 \mathrm{MHz}, \mathrm{C}_{6} \mathrm{D}_{6}\right)$ of $\mathbf{1 7 a}$. 


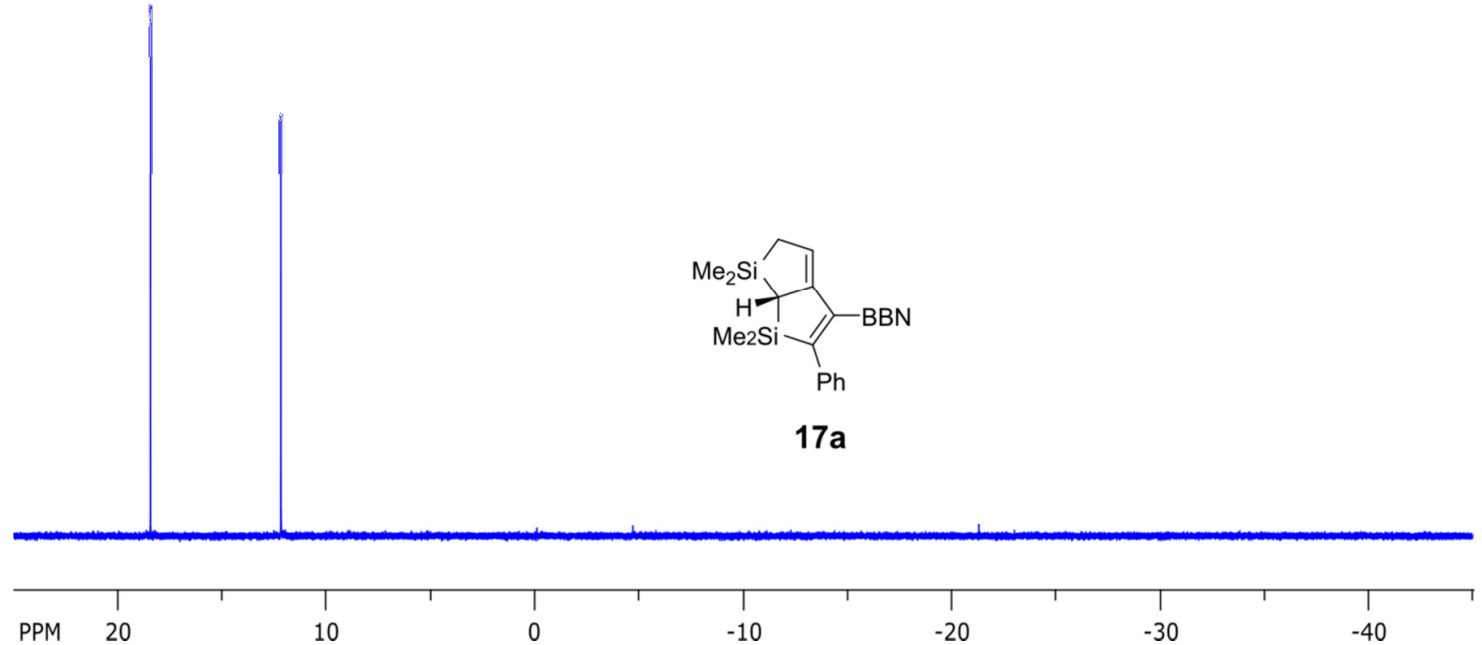

Figure S57. ${ }^{29} \mathrm{Si}\left\{{ }^{1} \mathrm{H}\right\}$ NMR spectrum (refocused INEPT, $119.2 \mathrm{MHz}, \mathrm{C}_{6} \mathrm{D}_{6}$ ) of $\mathbf{1 7 a}$.

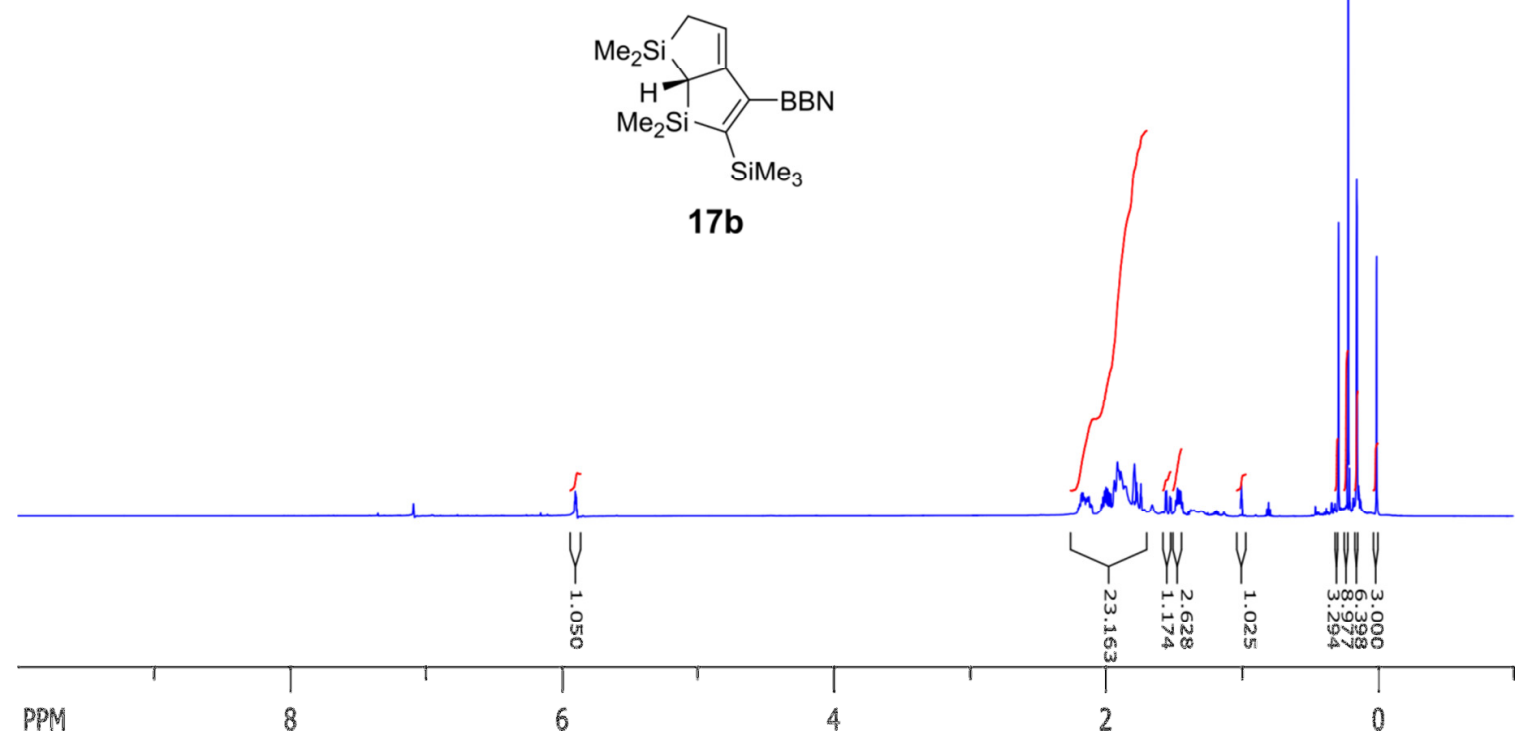

Figure S58. ${ }^{1} \mathrm{H}$ NMR spectrum $\left(600 \mathrm{MHz}, \mathrm{C}_{6} \mathrm{D}_{6}\right)$ of $\mathbf{1 7 b}$. 


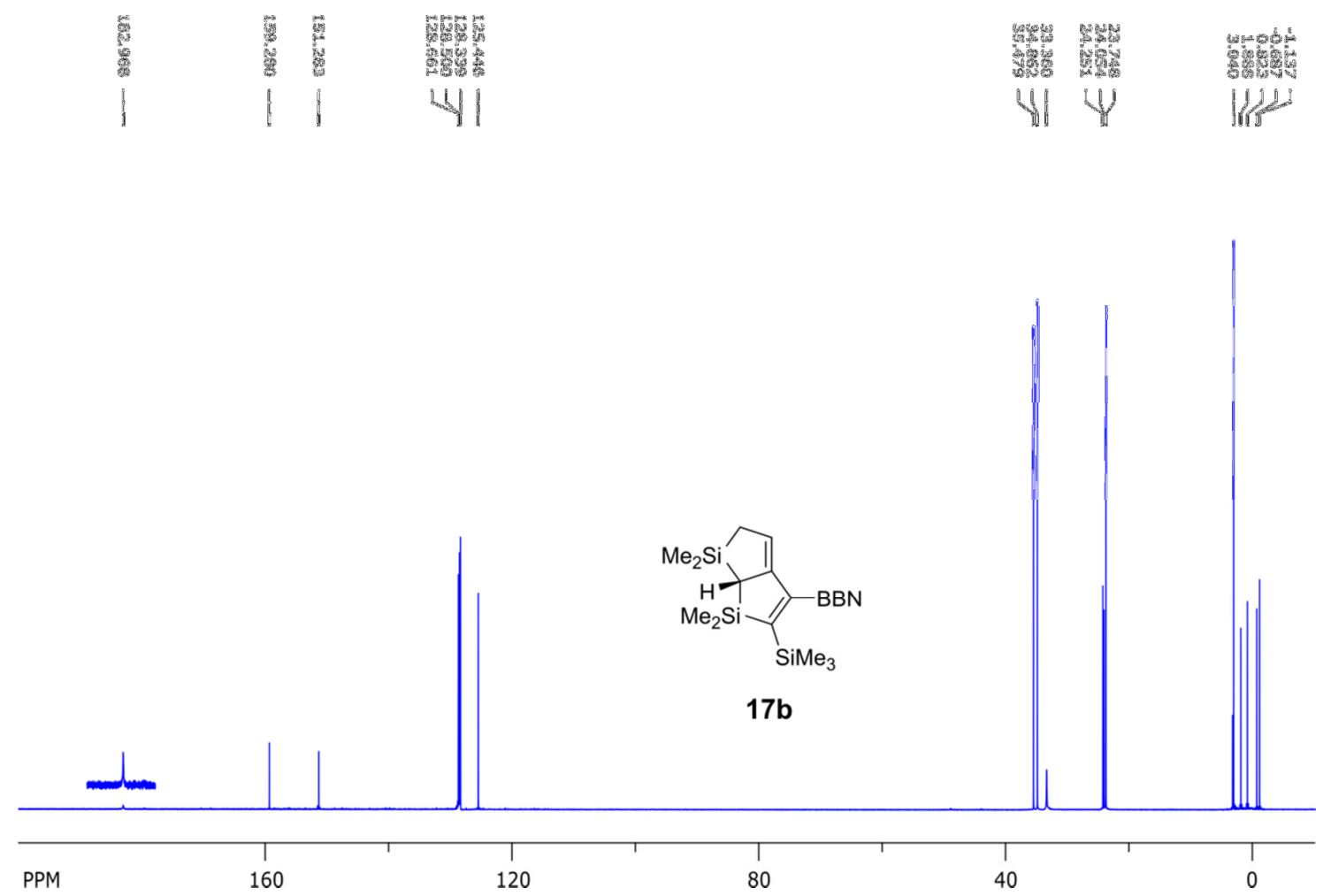

Figure S59. ${ }^{13} \mathrm{C}\left\{{ }^{1} \mathrm{H}\right\}$ NMR spectrum $\left(150.9 \mathrm{MHz}, \mathrm{C}_{6} \mathrm{D}_{6}\right)$ of $\mathbf{1 7 b}$.

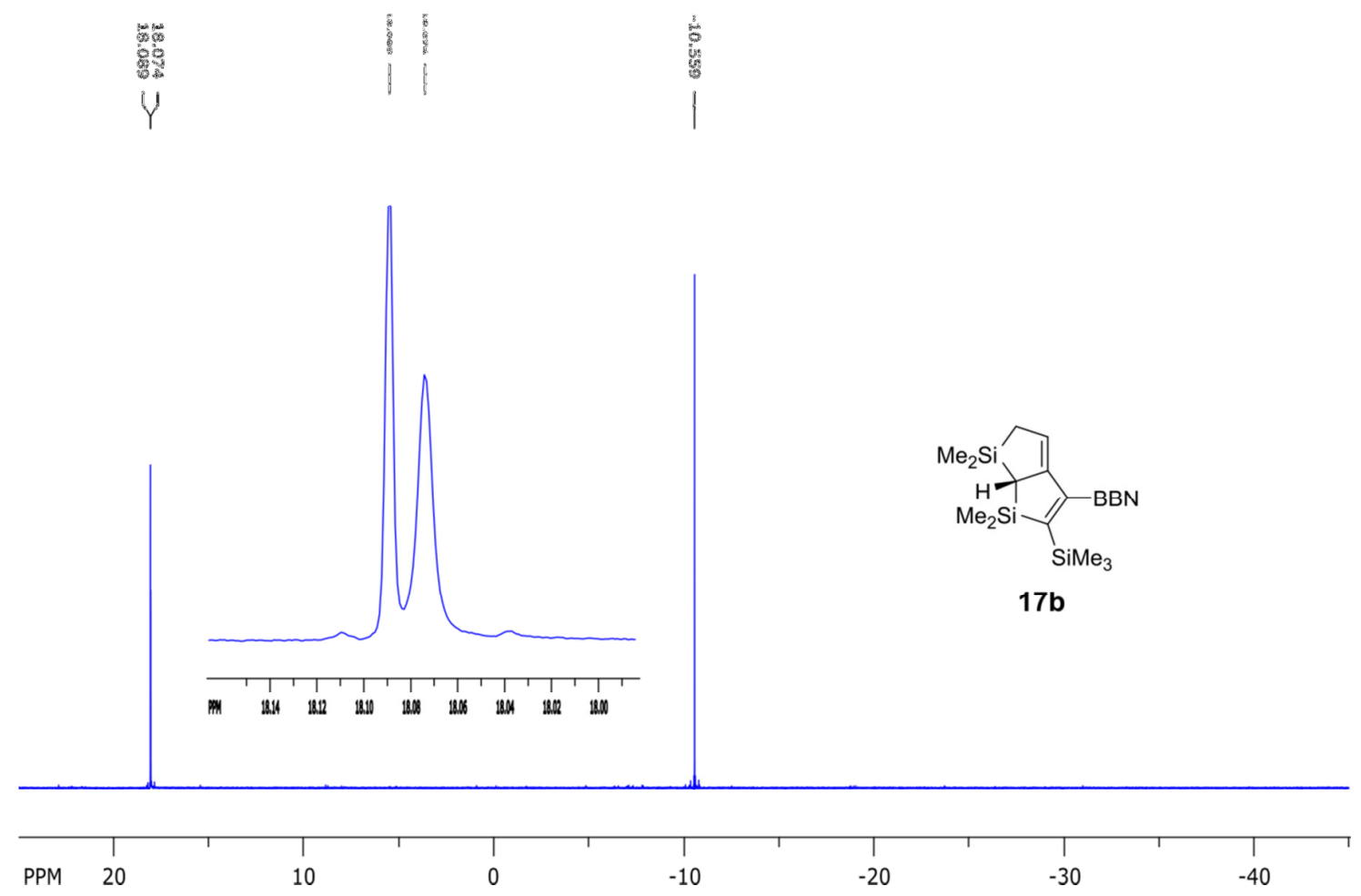

Figure S60. ${ }^{29} \mathrm{Si}\left\{{ }^{1} \mathrm{H}\right\}$ NMR spectrum (refocused INEPT, $119.2 \mathrm{MHz}, \mathrm{C}_{6} \mathrm{D}_{6}$ ) of $\mathbf{1 7 b}$. 


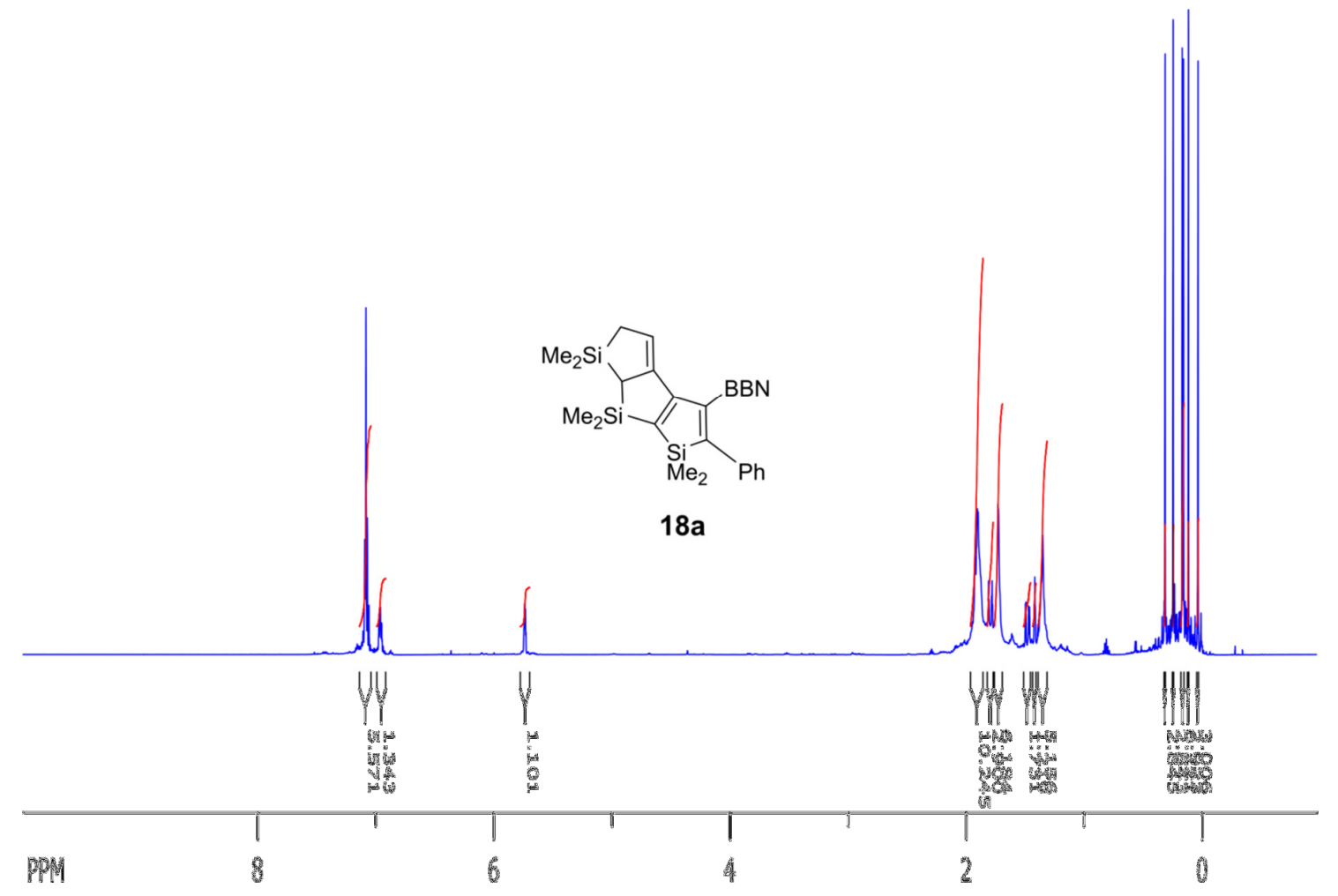

Figure S61. ${ }^{1} \mathrm{H}$ NMR spectrum $\left(600 \mathrm{MHz}, \mathrm{C}_{6} \mathrm{D}_{6}\right)$ of $\mathbf{1 8 a}$.

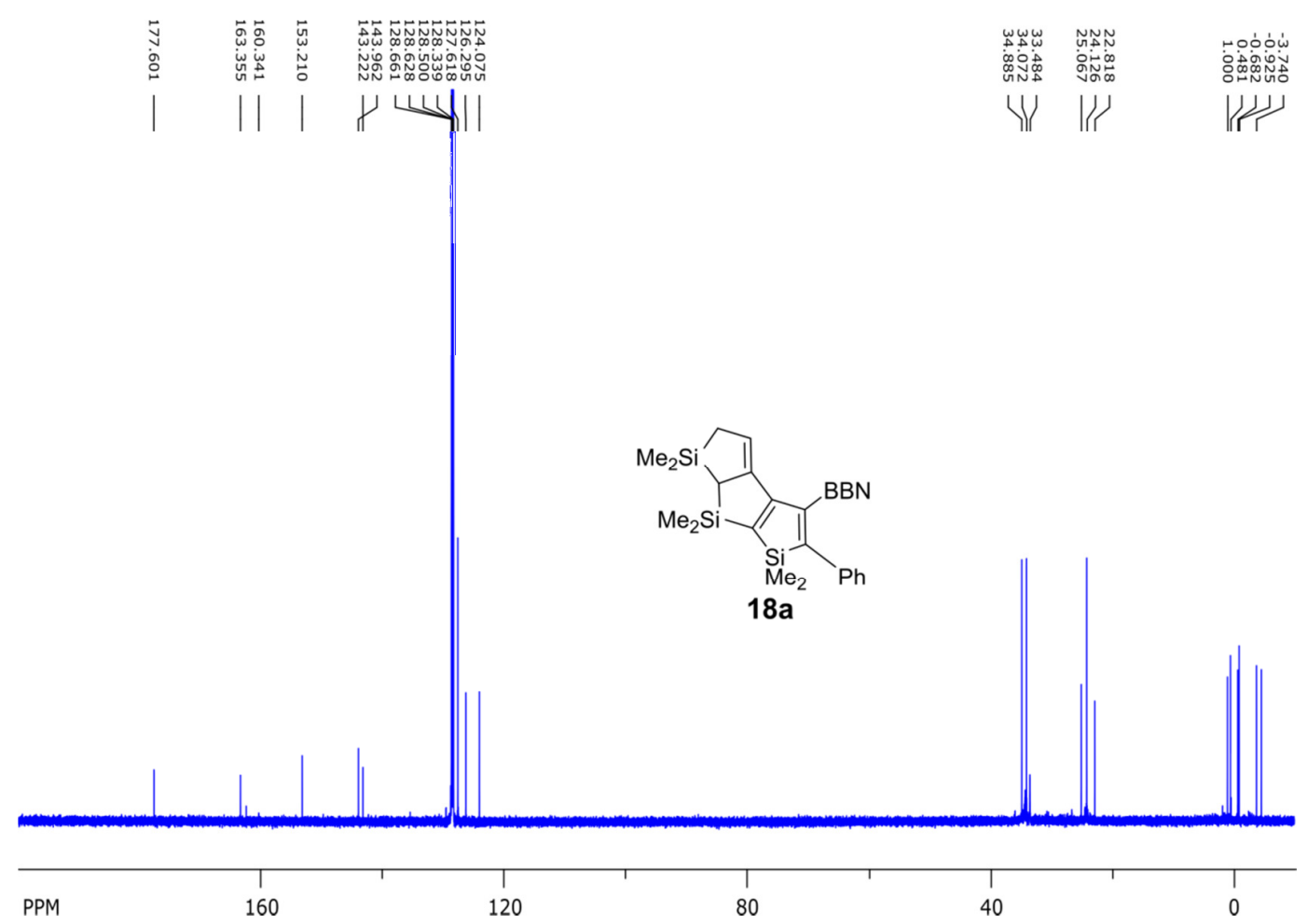

Figure S62. ${ }^{13} \mathrm{C}\left\{{ }^{1} \mathrm{H}\right\}$ NMR spectrum $\left(150.9 \mathrm{MHz}, \mathrm{C}_{6} \mathrm{D}_{6}\right)$ of 18 . 


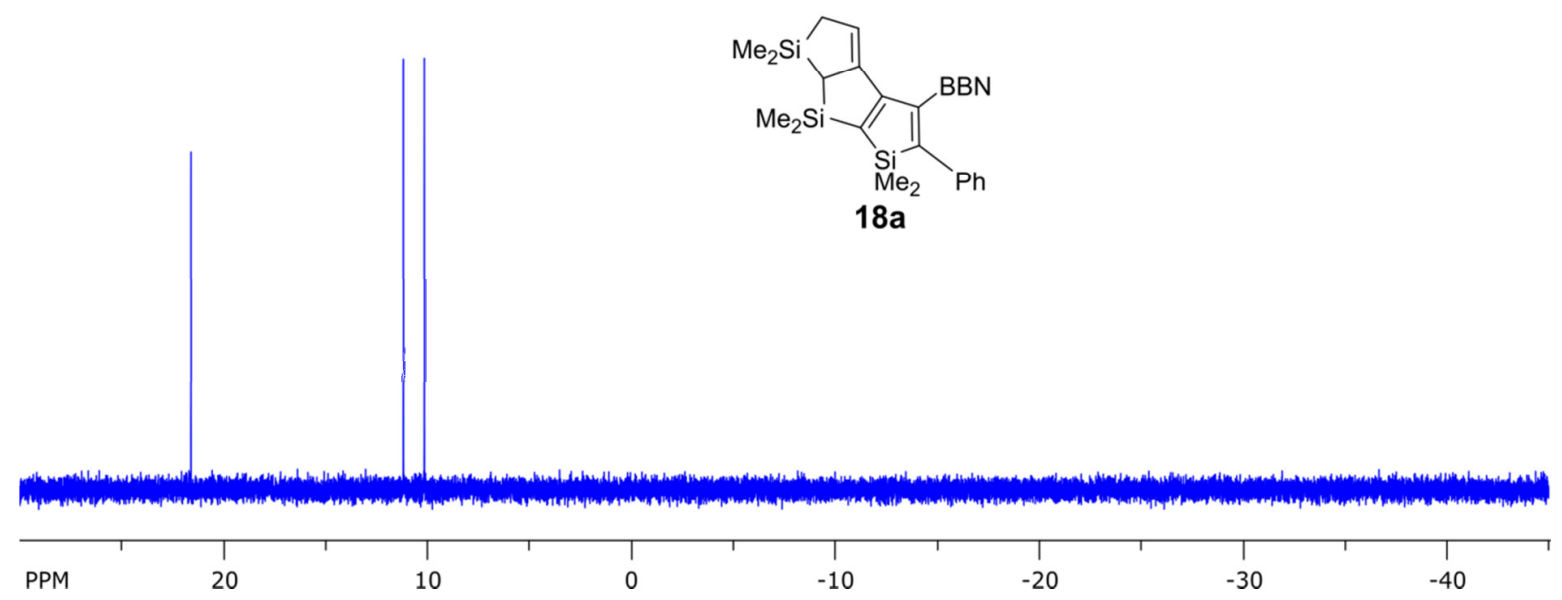

Figure S63. ${ }^{29} \mathrm{Si}\left\{{ }^{1} \mathrm{H}\right\}$ NMR spectrum (refocused INEPT, 119.2 MHz, $\mathrm{C}_{6} \mathrm{D}_{6}$ ) of 18a.

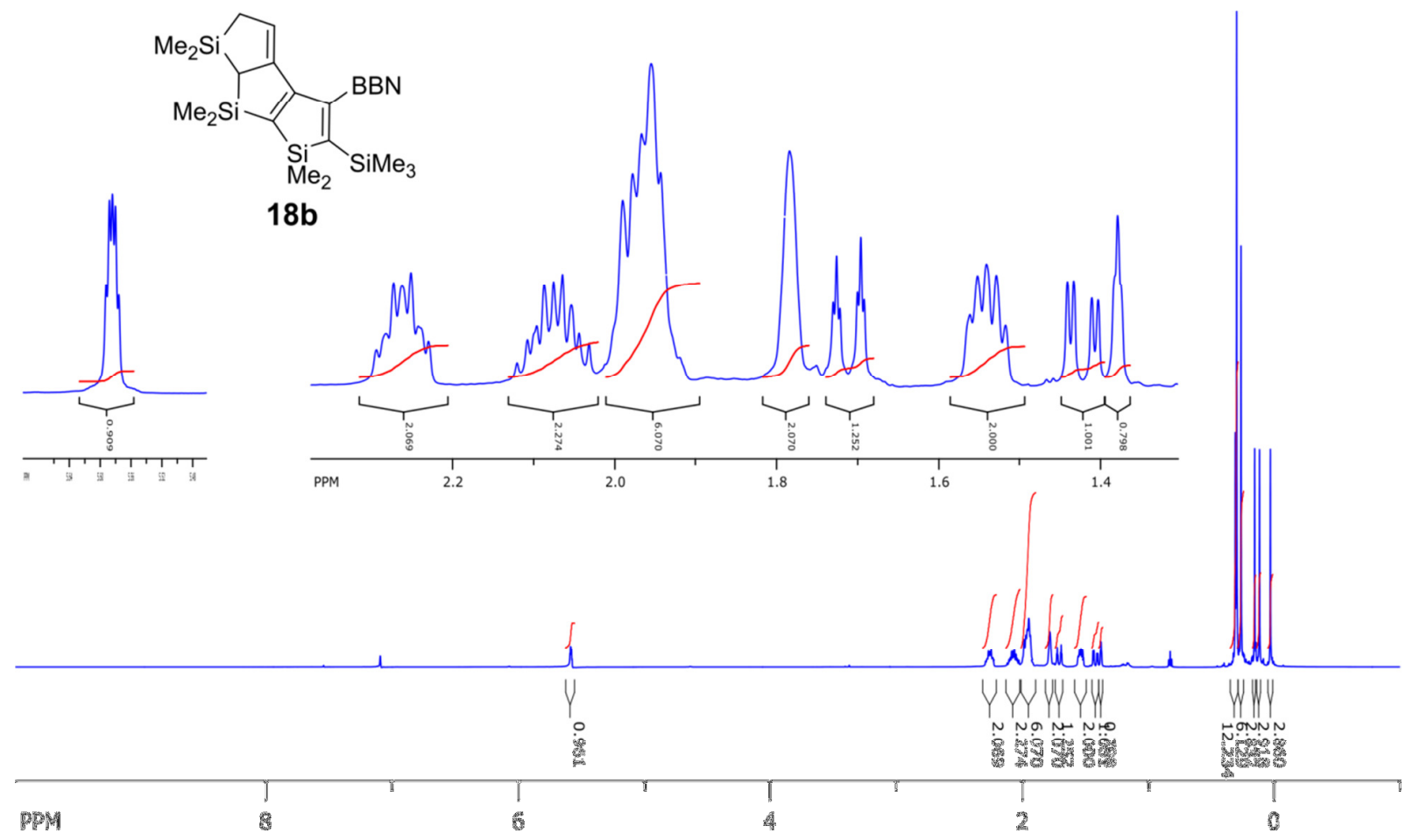

Figure S64. ${ }^{1} \mathrm{H}$ NMR spectrum $\left(600 \mathrm{MHz}, \mathrm{C}_{6} \mathrm{D}_{6}\right)$ of $\mathbf{1 8 b}$. 


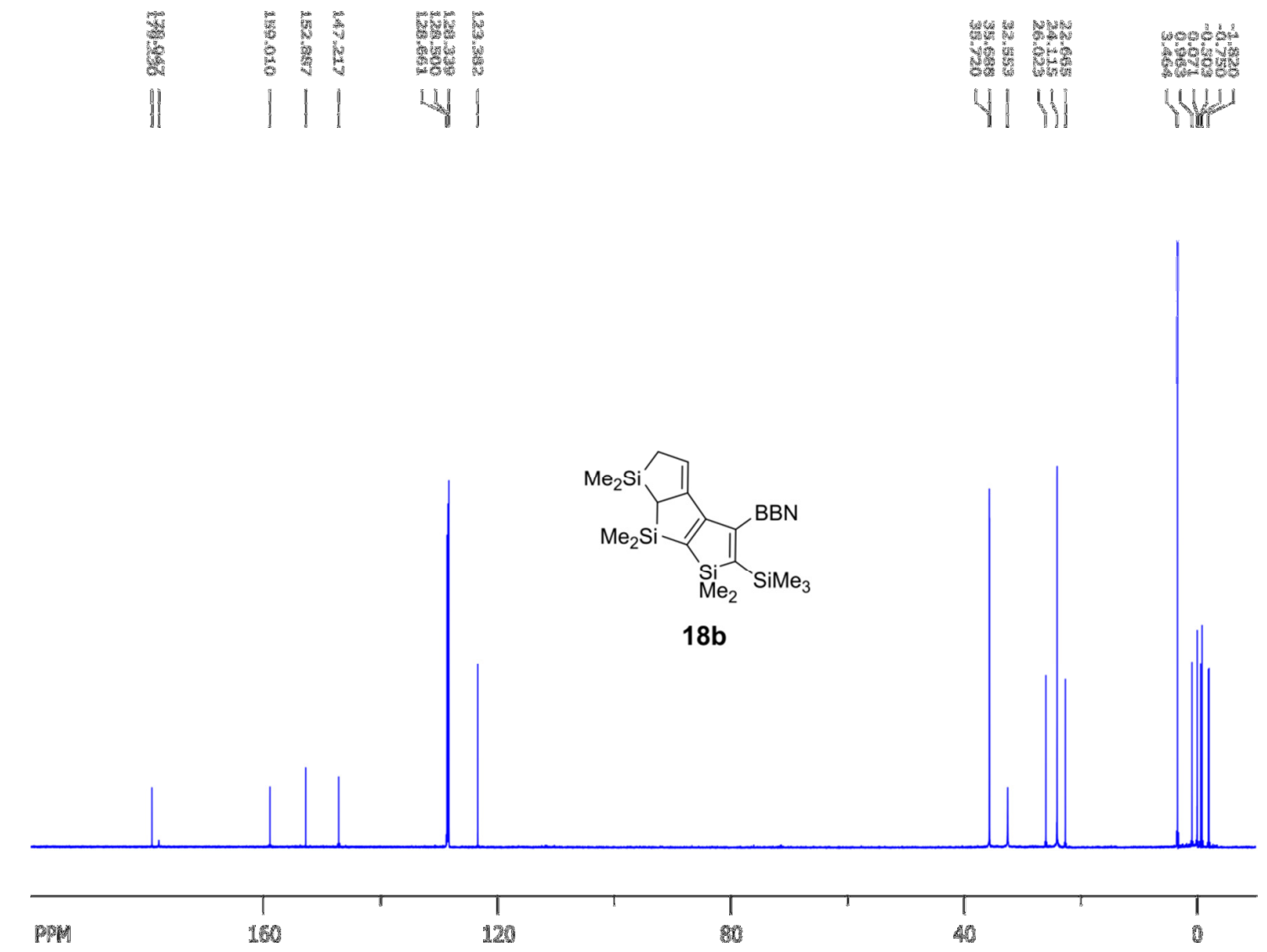

Figure S65. ${ }^{13} \mathrm{C}\left\{{ }^{1} \mathrm{H}\right\}$ NMR spectrum $\left(150.9 \mathrm{MHz}, \mathrm{C}_{6} \mathrm{D}_{6}\right)$ of $\mathbf{1 8 b}$.
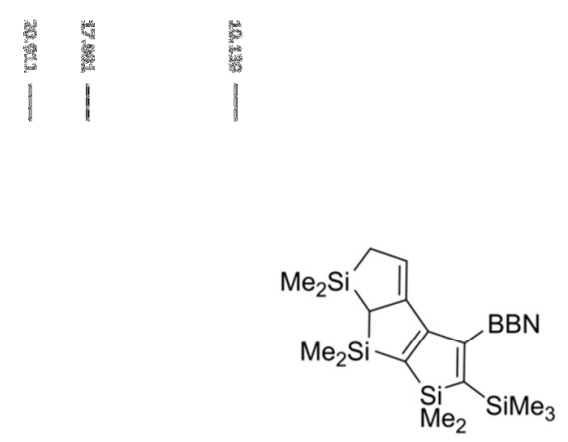

$18 b$

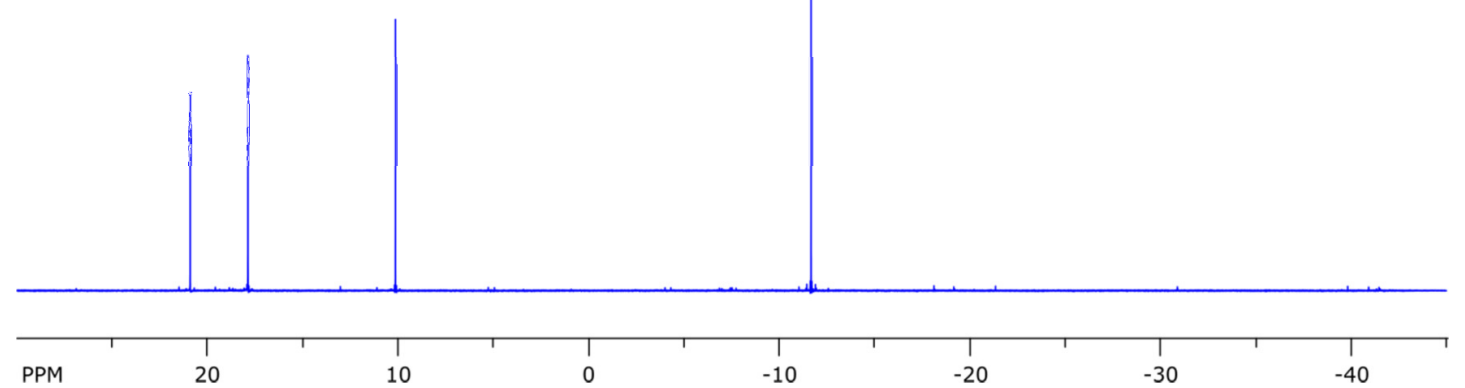

Figure S66. ${ }^{29} \mathrm{Si}\left\{{ }^{1} \mathrm{H}\right\}$ NMR spectrum (refocused INEPT, 119.2 MHz, $\mathrm{C}_{6} \mathrm{D}_{6}$ ) of $\mathbf{1 8 b}$. 


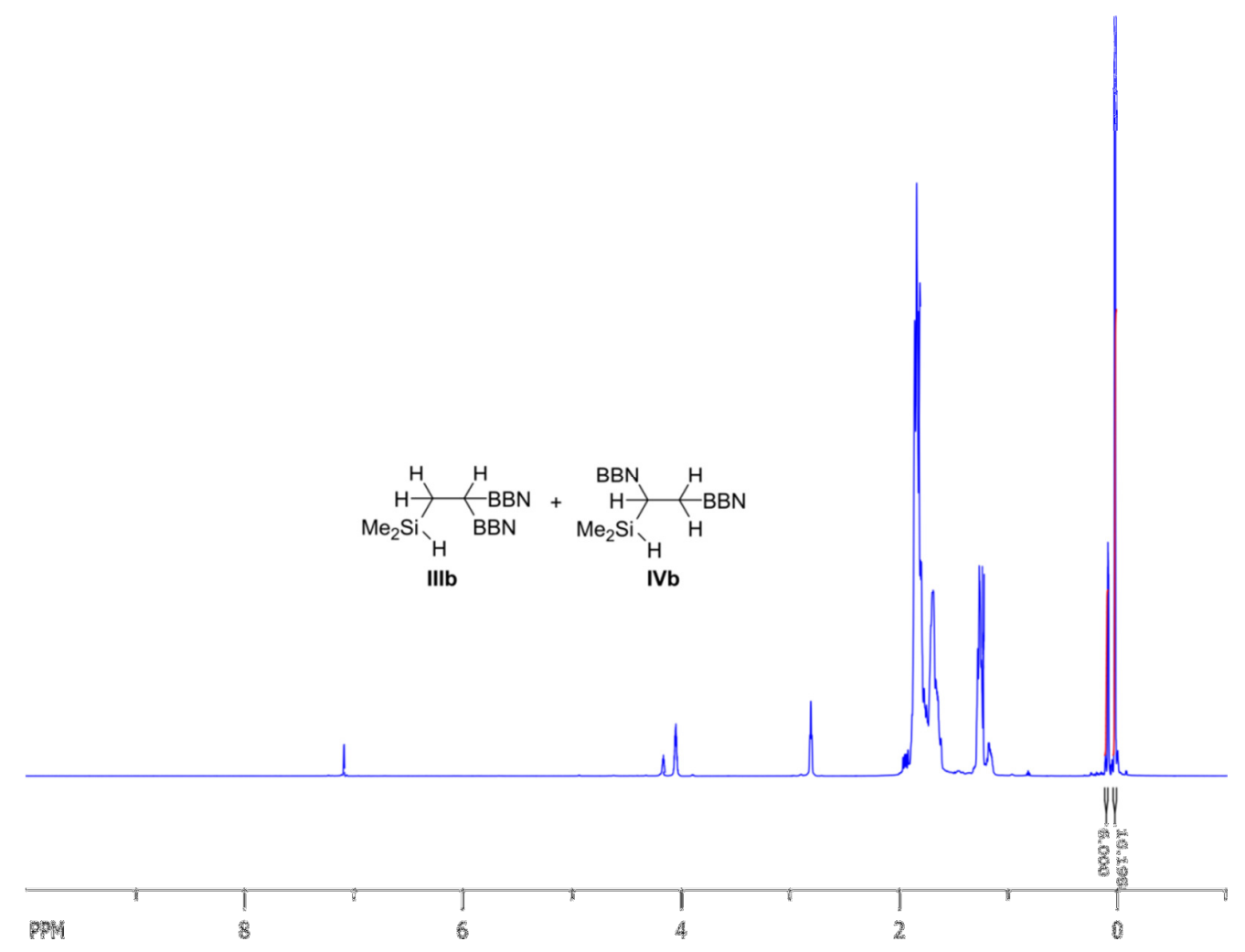

Figure S67. ${ }^{1} \mathrm{H}$ NMR spectrum $\left(600 \mathrm{MHz}, \mathrm{C}_{6} \mathrm{D}_{6}\right)$ of the mixture IIIb and IVb.

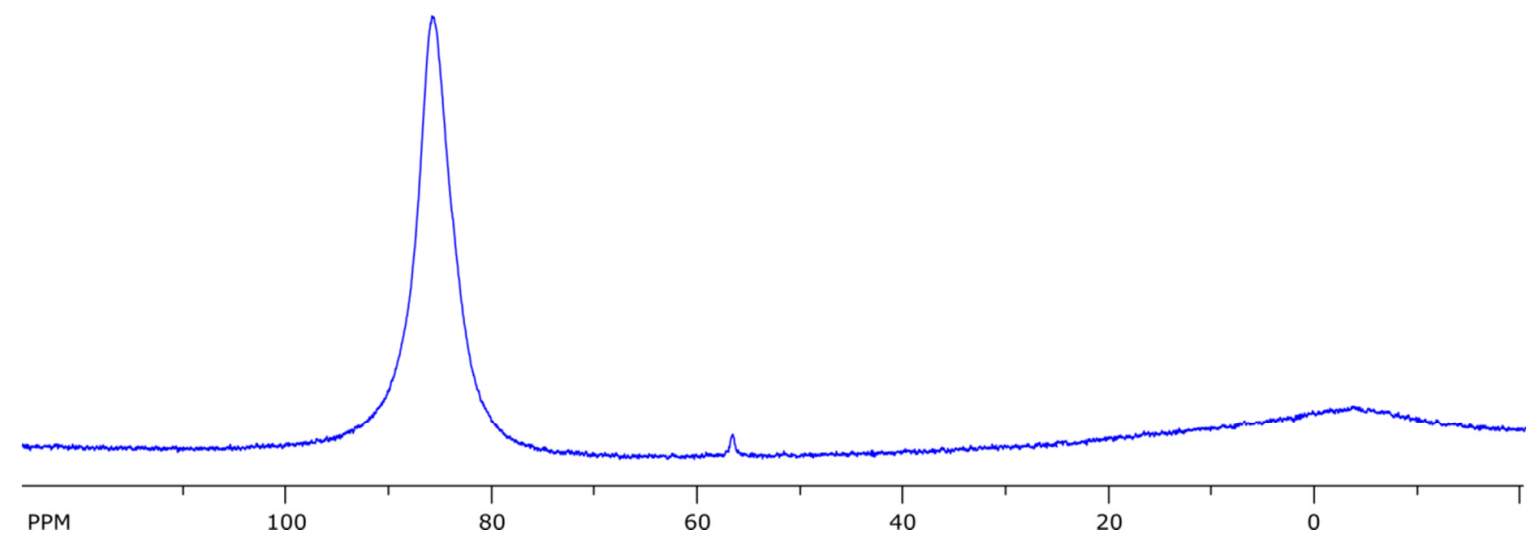

Figure S68. ${ }^{11} \mathrm{~B}$ NMR spectrum $\left(192.6 \mathrm{MHz}, \mathrm{C}_{6} \mathrm{D}_{6}\right)$ of the mixture IIIb and IVb. 


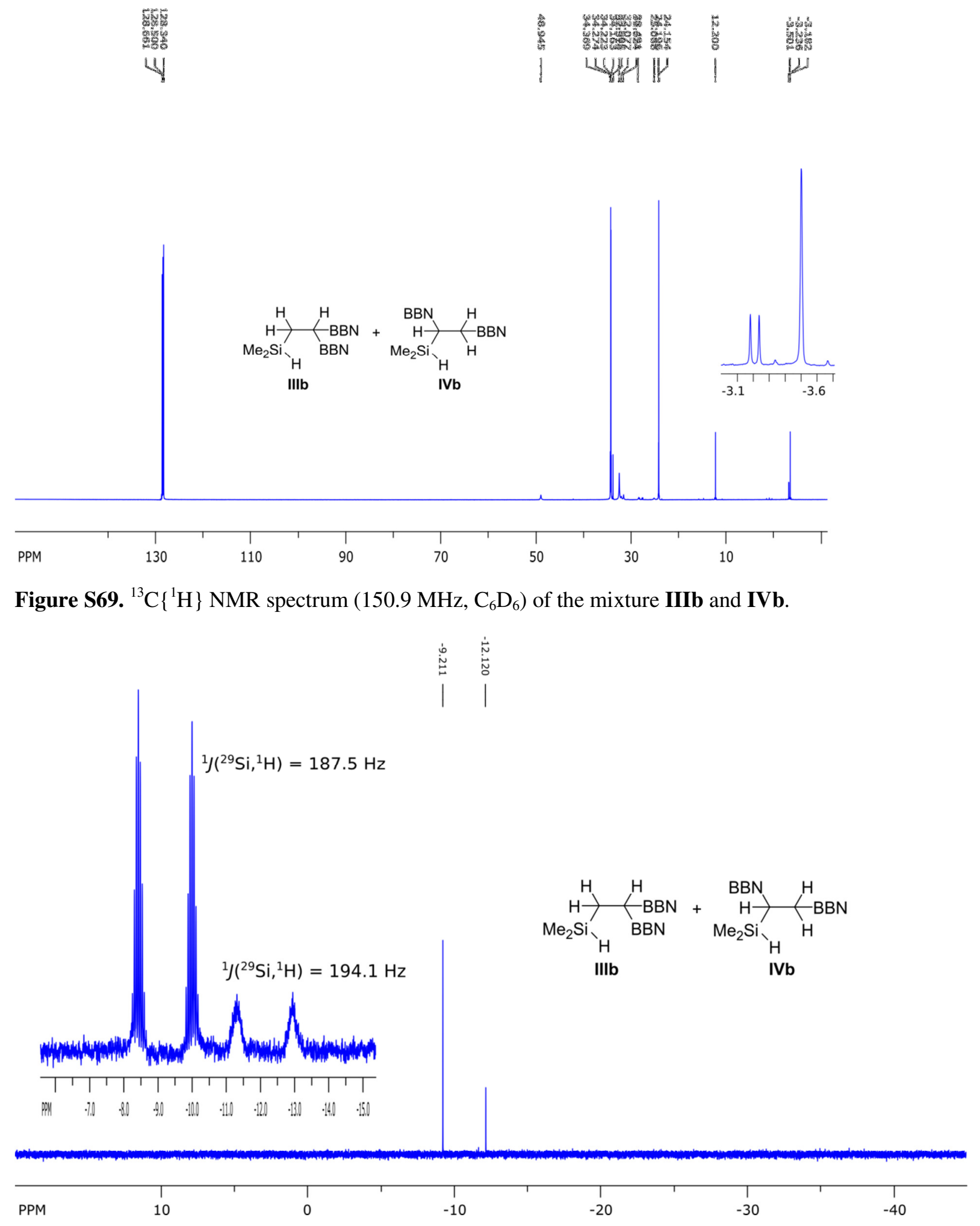

Figure S70. ${ }^{29} \mathrm{Si}\left\{{ }^{1} \mathrm{H}\right\}$ and ${ }^{29} \mathrm{Si}$ (expansion) NMR spectra (refocused INEPT based on ${ }^{1} J\left({ }^{29} \mathrm{~S},{ }^{1} \mathrm{H}\right)=$ $190 \mathrm{~Hz}, 119.2 \mathrm{MHz}, \mathrm{C}_{6} \mathbf{D}_{6}$ ) of the mixture of IIIb and $\mathbf{I V b}$. 


\section{X-ray crystallographic analysis details.}

The diffraction data of compound 7d was collected on the Super Nova diffractometer of Rigaku Oxford Diffraction equipped with mirror-collimated CuK $\alpha$ radiation (1.54184 $\AA$ ) from the sealed micro-focus Xray tube, CCD detector Atlas S2, and the Cryostream $800+$ chiller. The structure was solved by charge flipping using the Superflip program, and refined by full-matrix least squares on $\mathrm{F}^{2}$ with the program Jana2006. ${ }^{[1]}$

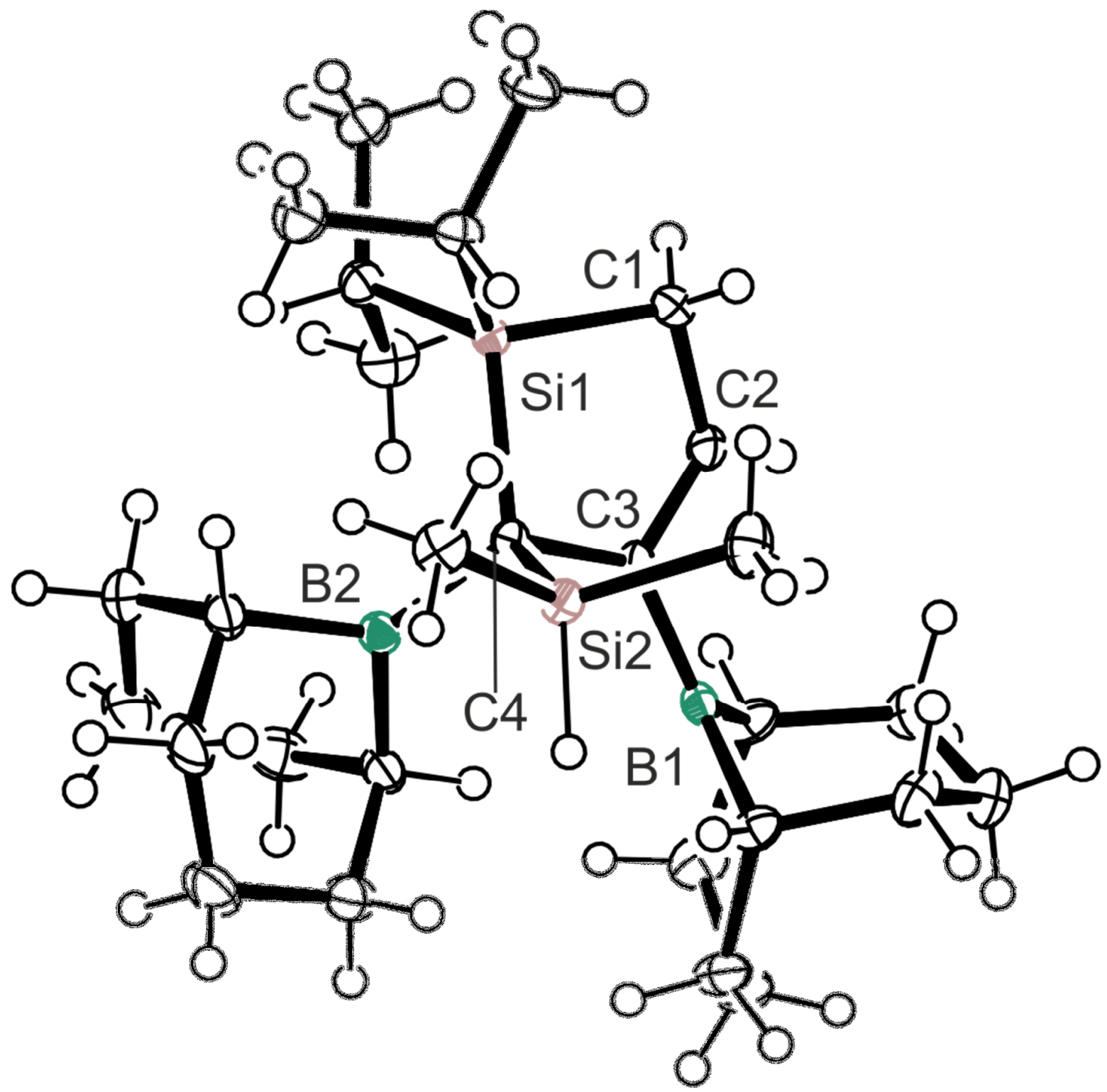

Figure S71. Molecular structure of 3,4-diboryl-2,5-dihydrosilole 7d with $50 \%$ probability ellipsoids. 
Table S1. Crystal data and structure refinement.

Identification code

CCDC Number

Formula

Formula weight

Size

Crystal morphology

Temperature

Wavelength

Crystal system

Space group

Unit cell dimensions

Volume

Z

Density (calculated)

Absorption coefficient

$F(000)$

Data collection range

Index ranges

Reflections collected

Independent reflections

Observed reflections

Absorption correction

Max. and min. transmission

Refinement method

Data / constraints / parameters

Goodness of fit

$R$ indices (all data)

\section{Compound 7d}

2041809

$\mathrm{C}_{28} \mathrm{H}_{52} \mathrm{~B}_{2} \mathrm{Si}_{2}$

466.5

$0.412 \times 0.296 \times 0.134 \mathrm{~mm}$

prismatic

120(2) K

$1.54184 \AA\left[\mathrm{Cu}-K_{\alpha}\right]$

triclinic

$\mathrm{P}-1$

$a=9.7621(2) \AA \quad \alpha=94.663(1)^{\circ}$

$b=10.1778(2) \AA \quad \beta=107.294(1)^{\circ}$

$c=16.3576(2) \AA \quad \gamma=111.476(1)^{\circ}$

$1410.58(5) \AA^{3}$

2

$1.0984 \mathrm{Mg} / \mathrm{m}^{3}$

$1.216 \mathrm{~mm}^{-1}$

516

$4.78 \leq \theta \leq 67.36^{\circ}$

$-11 \leq h \leq 11,-12 \leq k \leq 12,-19 \leq l \leq 19$

30520

5043

$4784[I>3 \sigma(I)]$

analytical

0.715 and 0.885

Full

5043 / 205 / 292

2.375

$R_{1}=0.0338, w R_{2}=0.1131$ 


\section{DFT calculations details.}

Quantum mechanical calculations were performed by DFT using the Gaussian 09 package. ${ }^{[2]}$ The structures were initially optimized using standard methods at the STO-3G level. The final optimizations, including frequency analyses to confirm the true minima (i.e. no imaginary frequencies) were performed at the MO62X/6-31+G(d,p) level. Energies include Zero Point Energy correction.

DFT MO62X/6-31+G(d p) calculated Cartesian atomic coordinates.

\section{3,4-(BBN)2-1,1-dimethyl-5-trimethylsilyl-2,5-dihydrosilole (5b) $\mathbf{E}=-1607.971241$}

$\begin{array}{llcl}\text { C } & 1.6937332529 & -0.5864377785 & -0.4389889404 \\ \mathrm{C} & 0.874566987 & 0.4414116265 & -0.7949691337 \\ \mathrm{C} & -0.5076304878 & 0.1156113531 & -1.3724772784 \\ \mathrm{H} & -1.5106466059 & -1.8705848511 & -1.6298452304 \\ \mathrm{H} & -0.7242913218 & 0.7765894947 & -2.2239395479 \\ \mathrm{H} & -0.4204451781 & -1.4212492778 & -2.927318109 \\ \mathrm{~B} & -1.65631408 & 0.265916376 & -0.2840551845 \\ \mathrm{C} & -1.6088755363 & -0.4114593165 & 1.1407609339 \\ \mathrm{C} & -3.0717621438 & 0.9047234114 & -0.5606010498 \\ \mathrm{H} & -0.6234950727 & -0.8365079553 & 1.3744366978 \\ \mathrm{C} & -1.9240304352 & 0.6445942391 & 2.2282402596 \\ \mathrm{C} & -2.6364991987 & -1.5769971408 & 1.113282302 \\ \mathrm{C} & -3.4206037583 & 1.9370693319 & 0.539029329 \\ \mathrm{H} & -3.1269340366 & 1.4160946766 & -1.5315626738 \\ \mathrm{C} & -4.0555983782 & -0.2976605728 & -0.620712619 \\ \mathrm{H} & -1.9961467602 & 0.1486749021 & 3.2063427412 \\ \mathrm{H} & -1.064055362 & 1.3236833782 & 2.3018527299 \\ \mathrm{C} & -3.2028850073 & 1.4706419238 & 1.9927482113 \\ \mathrm{C} & -4.0464621478 & -1.1984283376 & 0.6247940962 \\ \mathrm{H} & -2.7059903737 & -2.0251432027 & 2.1148541481 \\ \mathrm{H} & -2.241822106 & -2.3661738905 & 0.4590621178 \\ \mathrm{H} & -2.8106386746 & 2.8325852886 & 0.3611585543 \\ \mathrm{H} & -4.4647162193 & 2.2593756445 & 0.4203222149 \\ \mathrm{H} & -3.8004593235 & -0.9045153603 & -1.5012908977 \\ \mathrm{H} & -5.074947265 & 0.0736092298 & -0.7941568518 \\ \mathrm{H} & -4.0699512658 & 0.896803004 & 2.3278254437 \\ \mathrm{H} & -3.1734727865 & 2.35420784 & 2.6416001856 \\ \mathrm{H} & -4.5986289709 & -2.1184499083 & 0.3985021766 \\ \mathrm{H} & -4.6035125667 & -0.7153106375 & 1.4298724508 \\ \mathrm{Si} & 3.3060190758 & -0.422428068 & 0.4930277903 \\ \mathrm{C} & 3.6059321252 & -2.0399617634 & 1.4206687104 \\ \mathrm{H} & 2.7819082652 & -2.2632043103 & 2.1067777063 \\ \mathrm{H} & 3.7058790363 & -2.8846564745 & 0.7291133863 \\ \mathrm{H} & 4.5288110121 & -1.9830638567 & 2.0078051119 \\ \mathrm{C} & 4.7853279685 & -0.1352352931 & -0.6434368224 \\ \mathrm{H} & 4.7802688363 & -0.854174481 & -1.4698252721 \\ & & & \end{array}$




$\begin{array}{cccc}\mathrm{H} & 4.7835538511 & 0.8709733825 & -1.0726193184 \\ \mathrm{H} & 5.7212055877 & -0.2641358806 & -0.0886314 \\ \mathrm{Si} & 0.9293446293 & -2.2091100112 & -0.9889705201 \\ \mathrm{C} & -0.5539649578 & -1.3773927467 & -1.8401002134 \\ \mathrm{C} & 3.1794917373 & 0.9659150914 & 1.7649566857 \\ \mathrm{H} & 3.0207454702 & 1.9407392964 & 1.2919351534 \\ \mathrm{H} & 2.3373629032 & 0.782621913 & 2.4419657996 \\ \mathrm{H} & 4.0920172433 & 1.0406345142 & 2.3666240529 \\ \mathrm{~B} & 1.169481613 & 1.9758534281 & -0.6420260422 \\ \mathrm{C} & 2.3001958415 & 2.7199514215 & -1.4443437105 \\ \mathrm{C} & 0.1613779012 & 3.0009193809 & 0.0049858757 \\ \mathrm{H} & 3.0721450928 & 2.0409199534 & -1.8248233976 \\ \mathrm{C} & 1.4804083339 & 3.2047268861 & -2.6827348949 \\ \mathrm{C} & 2.9810390549 & 3.8724771092 & -0.6744034381 \\ \mathrm{C} & -0.5631335849 & 3.6837612086 & -1.1939804466 \\ \mathrm{H} & -0.616217995 & 2.5201338267 & 0.6131693421 \\ \mathrm{C} & 0.9242298735 & 3.9880835876 & 0.9123900425 \\ \mathrm{H} & 2.1644976632 & 3.6752271072 & -3.4007701263 \\ \mathrm{H} & 1.0572242 & 2.3277515213 & -3.1945618645 \\ \mathrm{C} & 0.3491427959 & 4.181042512 & -2.3307669482 \\ \mathrm{C} & 2.062353517 & 4.7450460455 & 0.2088110554 \\ \mathrm{H} & 3.516763756 & 4.5226805994 & -1.3806607445 \\ \mathrm{H} & 3.7580138256 & 3.4286354673 & -0.0390064645 \\ \mathrm{H} & -1.2725033517 & 2.9576197013 & -1.6132869073 \\ \mathrm{H} & -1.1717129467 & 4.5177796902 & -0.8159841197 \\ \mathrm{H} & 1.3293186018 & 3.4276310116 & 1.7658356488 \\ \mathrm{H} & 0.2223466591 & 4.7191336009 & 1.336870435 \\ \mathrm{H} & 0.7800210716 & 5.1524601997 & -2.0782670715 \\ \mathrm{H} & -0.2643554045 & 4.3525693154 & -3.2230054701 \\ \mathrm{H} & 2.6776851761 & 5.2407858067 & 0.9686923967 \\ \mathrm{H} & 1.6317650249 & 5.5549075687 & -0.3851344151 \\ \mathrm{C} & 2.0344189791 & -3.1220998716 & -2.2179085094 \\ \mathrm{H} & 2.9368101851 & -3.504804573 & -1.7279361592 \\ \mathrm{H} & 1.5116234375 & -3.977198184 & -2.6604465509 \\ \mathrm{H} & 2.3463822931 & -2.4549549847 & -3.02752239 \\ \mathrm{C} & 0.4741119123 & -3.4451293481 & 0.3653827386 \\ \mathrm{H} & -0.0002326586 & -2.9728770013 & 1.2302572126 \\ \mathrm{H} & -0.2159937827 & -4.1995288896 & -0.0295678293 \\ \mathrm{H} & 1.3697071644 & -3.9686922091 & 0.7151850363 \\ & & & \\ & & & \end{array}$

4,5-(BBN) 2-1,1-dimethyl-5-trimethylsilyl-2,5-dihydrosilole (7b) $\mathbf{E}=-1607.979512$

$\begin{array}{llll}\text { C } & -0.6602928735 & 0.1231922092 & 0.4736418438 \\ \text { C } & -1.2404456865 & -1.2890854092 & 0.238524075 \\ \text { C } & -1.4129714331 & -2.0308044259 & 1.3623674524 \\ \text { H } & -1.8397169626 & -1.7604471232 & 3.4801984353 \\ \text { H } & -0.124367696 & -1.7993019688 & 3.0619802591 \\ \text { Si } & -1.3692222231 & 1.5926013787 & -0.5366200752\end{array}$




\begin{tabular}{|c|c|c|c|}
\hline $\mathrm{C}$ & -3.2357192967 & 1.3897335752 & -0.7765218295 \\
\hline $\mathrm{H}$ & -3.556068433 & 0.3707527904 & -0.5375618679 \\
\hline $\mathrm{H}$ & -3.7904024024 & 2.0770083001 & -0.1306600733 \\
\hline $\mathrm{H}$ & -3.5177156455 & 1.5950211754 & -1.8146214594 \\
\hline $\mathrm{C}$ & -1.0269983524 & 3.2169130217 & 0.3726257107 \\
\hline $\mathrm{H}$ & -1.5447938246 & 3.3035504572 & 1.3321989755 \\
\hline $\mathrm{H}$ & 0.0453831252 & 3.3559298197 & 0.5496301517 \\
\hline $\mathrm{H}$ & -1.3652735642 & 4.0457718097 & -0.260137627 \\
\hline $\mathrm{Si}$ & -1.1334527619 & 0.3928706036 & 2.3110649752 \\
\hline $\mathrm{C}$ & -1.1117648071 & -1.4506267938 & 2.723048522 \\
\hline $\mathrm{C}$ & -0.5161758444 & 1.8410497249 & -2.2092109289 \\
\hline $\mathrm{H}$ & 0.501058101 & 2.2250908478 & -2.0713747214 \\
\hline $\mathrm{H}$ & -0.4513518777 & 0.9361849763 & -2.8183339356 \\
\hline $\mathrm{H}$ & -1.0726300189 & 2.5930473277 & -2.7813907742 \\
\hline B & 0.8539909735 & 0.162690019 & 0.0710039332 \\
\hline $\mathrm{C}$ & 1.5003252611 & -0.8678715694 & -0.9437985385 \\
\hline $\mathrm{C}$ & 1.9847709721 & 1.0526087302 & 0.7277059246 \\
\hline $\mathrm{H}$ & 0.7505463936 & -1.4393902451 & -1.5097726679 \\
\hline $\mathrm{C}$ & 2.427017832 & -0.1974934814 & -1.9801342805 \\
\hline $\mathrm{C}$ & 2.2249956455 & -1.8802074455 & -0.0160624259 \\
\hline $\mathrm{C}$ & 2.8900556761 & 1.7334199218 & -0.3196841653 \\
\hline $\mathrm{H}$ & 1.6009923964 & 1.8360283543 & 1.3924871425 \\
\hline $\mathrm{C}$ & 2.7345120531 & 0.0254732127 & 1.6326881534 \\
\hline $\mathrm{H}$ & 2.9638148292 & -0.9710518994 & -2.5479968884 \\
\hline $\mathrm{H}$ & 1.7914624182 & 0.3219813431 & -2.7082047863 \\
\hline $\mathrm{C}$ & 3.4458293677 & 0.8117339868 & -1.4187932483 \\
\hline $\mathrm{C}$ & 3.2578199168 & -1.2349713114 & 0.9225132571 \\
\hline $\mathrm{H}$ & 2.7118453515 & -2.6568205679 & -0.6226564569 \\
\hline $\mathrm{H}$ & 1.4628725344 & -2.3937162414 & 0.589701701 \\
\hline $\mathrm{H}$ & 2.3069668765 & 2.5362815095 & -0.7922298332 \\
\hline $\mathrm{H}$ & 3.728896778 & 2.233912342 & 0.1841033835 \\
\hline $\mathrm{H}$ & 2.0446098043 & -0.3030466916 & 2.4236796773 \\
\hline $\mathrm{H}$ & 3.5634610279 & 0.5329717046 & 2.1455327419 \\
\hline $\mathrm{H}$ & 4.3250976279 & 0.2839243238 & -1.042750988 \\
\hline $\mathrm{H}$ & 3.8140677803 & 1.4329034175 & -2.2440378589 \\
\hline $\mathrm{H}$ & 3.5524556145 & -1.9669445336 & 1.6840551843 \\
\hline $\mathrm{H}$ & 4.1701540547 & -1.0043734073 & 0.369376446 \\
\hline $\mathrm{H}$ & -1.753696267 & -3.0628512314 & 1.2942817279 \\
\hline B & -1.5398693178 & -2.0011563259 & -1.1272020071 \\
\hline $\mathrm{C}$ & -2.0788413369 & -1.3593722447 & -2.4697558223 \\
\hline $\mathrm{C}$ & -1.4470362111 & -3.577631607 & -1.2942376078 \\
\hline $\mathrm{H}$ & -2.1521736353 & -0.269259167 & -2.4469617218 \\
\hline $\mathrm{C}$ & -1.1587596014 & -1.7273875194 & -3.6589639536 \\
\hline $\mathrm{C}$ & -3.5346561317 & -1.8826223528 & -2.6154881161 \\
\hline $\mathrm{C}$ & -0.4801418857 & -3.9064379019 & -2.4577839859 \\
\hline $\mathrm{H}$ & -1.0425236447 & -4.0760209531 & -0.4048761322 \\
\hline $\mathrm{C}$ & -2.8887786552 & -4.1088748595 & -1.5065191399 \\
\hline $\mathrm{H}$ & -1.6122213436 & -1.3764884811 & -4.5968531731 \\
\hline $\mathrm{H}$ & -0.2232612927 & -1.1601074332 & -3.5465810526 \\
\hline $\mathrm{C}$ & -0.8009830392 & -3.2175309481 & -3.7931547865 \\
\hline $\mathrm{C}$ & -3.6863848642 & -3.4111082443 & -2.6204544036 \\
\hline
\end{tabular}




$\begin{array}{lcll}\mathrm{H} & -3.9796002198 & -1.4660019265 & -3.529806066 \\ \mathrm{H} & -4.1266231709 & -1.4789668051 & -1.7809396389 \\ \mathrm{H} & 0.5335819554 & -3.61672371 & -2.1451379535 \\ \mathrm{H} & -0.4481510293 & -4.9940895816 & -2.6112821615 \\ \mathrm{H} & -3.4342115998 & -3.9799521501 & -0.5601475957 \\ \mathrm{H} & -2.8608135328 & -5.1909520182 & -1.6974880426 \\ \mathrm{H} & -1.6086052257 & -3.7516178164 & -4.2985832606 \\ \mathrm{H} & 0.0675746777 & -3.3104353034 & -4.4562511535 \\ \mathrm{H} & -4.748199703 & -3.6598227954 & -2.5044204947 \\ \mathrm{H} & -3.3990650706 & -3.8095388169 & -3.5958190701 \\ \mathrm{C} & -2.9322934121 & 0.9394583981 & 2.5156359932 \\ \mathrm{H} & -3.6027638003 & 0.290511223 & 1.9431985711 \\ \mathrm{H} & -3.2013401374 & 0.8470985294 & 3.5742911287 \\ \mathrm{H} & -3.1208306056 & 1.9741837611 & 2.2166275263 \\ \mathrm{C} & -0.0915112937 & 1.4977995001 & 3.4273368126 \\ \mathrm{H} & 0.9359680937 & 1.1495459431 & 3.5509367433 \\ \mathrm{H} & -0.0607232072 & 2.5275435148 & 3.0587345492 \\ \mathrm{H} & -0.5631479598 & 1.5151917751 & 4.4165514833\end{array}$

\section{3,4-(BBN) $)_{2}$-1,1-dimethyl-5-phenyl-2,3-dihydrosilole (5a) $\mathbf{E}=-1430.382214$}

$\begin{array}{llcl}\text { C } & -0.9278334759 & 1.5164144032 & -0.3133366884 \\ \mathrm{C} & -0.4649531773 & 0.2836755613 & -0.6492317885 \\ \mathrm{C} & 0.8789159156 & 0.1929051714 & -1.3640078457 \\ \mathrm{H} & 2.3708053661 & 1.8129395767 & -1.771856096 \\ \mathrm{H} & 0.8102682198 & -0.5135013458 & -2.2034458578 \\ \mathrm{H} & 1.114747247 & 1.6513770261 & -2.98135845 \\ \mathrm{~B} & 2.0166351177 & -0.2867836562 & -0.3625748521 \\ \mathrm{C} & 2.3157841732 & 0.4214988461 & 1.0158294699 \\ \mathrm{C} & 3.1256034147 & -1.3516382877 & -0.7078368465 \\ \mathrm{H} & 1.5417218096 & 1.1480514987 & 1.2970545455 \\ \mathrm{C} & 2.3779293125 & -0.6438468476 & 2.1358251163 \\ \mathrm{C} & 3.6516381883 & 1.1973525017 & 0.8387221178 \\ \mathrm{C} & 3.2935392943 & -2.3856176349 & 0.4305350894 \\ \mathrm{H} & 2.9156845975 & -1.9014581067 & -1.6354409854 \\ \mathrm{C} & 4.399045346 & -0.4941620102 & -0.9614145642 \\ \mathrm{H} & 2.6627045611 & -0.1632083149 & 3.0820478325 \\ \mathrm{H} & 1.363133828 & -1.0341691636 & 2.2941171866 \\ \mathrm{C} & 3.3383836116 & -1.8168151594 & 1.8648199058 \\ \mathrm{C} & 4.8123538055 & 0.3926291738 & 0.2237819507 \\ \mathrm{H} & 3.9657311134 & 1.607955926 & 1.8090294669 \\ \mathrm{H} & 3.4555666747 & 2.0665910096 & 0.1959314269 \\ \mathrm{H} & 2.456637989 & -3.0925150502 & 0.3585890272 \\ \mathrm{H} & 4.2010768036 & -2.9805298533 & 0.2553086327 \\ \mathrm{H} & 4.2195516458 & 0.1426613567 & -1.8398346698 \\ \mathrm{H} & 5.2345553843 & -1.1540084999 & -1.2318087333 \\ \mathrm{H} & 4.3576228386 & -1.506908095 & 2.1077350246 \\ \mathrm{H} & 3.1088357624 & -2.627151007 & 2.5672153068 \\ \mathrm{H} & 5.5854819268 & 1.0942310291 & -0.1120955434\end{array}$




$\begin{array}{llll}\mathrm{H} & 5.2914429058 & -0.2225229979 & 0.9885215745 \\ \mathrm{Si} & 0.1792989174 & 2.8571715967 & -1.0236168001 \\ \mathrm{C} & 1.3025383239 & 1.6037719633 & -1.9023596143 \\ \mathrm{~B} & -1.2010489514 & -1.0550104017 & -0.3076508576 \\ \mathrm{C} & -2.6571753319 & -1.3957021706 & -0.7921120884 \\ \mathrm{C} & -0.4695705472 & -2.3337805835 & 0.2544537482 \\ \mathrm{H} & -3.2175167758 & -0.5046094466 & -1.1009059765 \\ \mathrm{C} & -2.360423154 & -2.2261739061 & -2.0784120487 \\ \mathrm{C} & -3.4866708945 & -2.1531972022 & 0.2655410234 \\ \mathrm{C} & -0.28083205 & -3.2735525284 & -0.9715129337 \\ \mathrm{H} & 0.5338536661 & -2.1230695779 & 0.6470110341 \\ \mathrm{C} & -1.3016336126 & -2.9442084973 & 1.4018314073 \\ \mathrm{H} & -3.3088958622 & -2.4904730123 & -2.5646729075 \\ \mathrm{H} & -1.8221866601 & -1.5836859957 & -2.7917457078 \\ \mathrm{C} & -1.5368202471 & -3.5004668804 & -1.8342274904 \\ \mathrm{C} & -2.7543513358 & -3.2755729176 & 1.0278086353 \\ \mathrm{H} & -4.3937878344 & -2.5673415214 & -0.197001954 \\ \mathrm{H} & -3.82947806 & -1.4075308718 & 0.9940902404 \\ \mathrm{H} & 0.5034756014 & -2.8367796864 & -1.6067595351 \\ \mathrm{H} & 0.113233509 & -4.2413470581 & -0.6292759689 \\ \mathrm{H} & -1.3031824495 & -2.2318178278 & 2.2403463052 \\ \mathrm{H} & -0.808852079 & -3.851568955 & 1.7780389334 \\ \mathrm{H} & -2.1741237621 & -4.2630677077 & -1.3814354031 \\ \mathrm{H} & -1.2262475005 & -3.9134165214 & -2.8013752253 \\ \mathrm{H} & -3.3124270701 & -3.4951272366 & 1.945851167 \\ \mathrm{H} & -2.7722930514 & -4.2034226671 & 0.4510884586 \\ \mathrm{C} & -0.6902300907 & 4.0010644524 & -2.2455345021 \\ \mathrm{H} & -1.3472654486 & 4.7119712319 & -1.7337041394 \\ \mathrm{H} & 0.047026417 & 4.583837449 & -2.8091587823 \\ \mathrm{H} & -1.289880209 & 3.429432828 & -2.9607734747 \\ \mathrm{C} & 1.0054993545 & 3.9414159181 & 0.2769433549 \\ \mathrm{H} & 1.546159315 & 3.3581429579 & 1.0270519717 \\ \mathrm{H} & 1.7093997708 & 4.6407249464 & -0.1873328178 \\ \mathrm{H} & 0.2436030308 & 4.5326903975 & 0.7971920423 \\ \mathrm{C} & -2.1085119011 & 1.6902727029 & 0.5544955544 \\ \mathrm{C} & -3.0960533127 & 2.6468533393 & 0.2700175259 \\ \mathrm{C} & -2.2699481082 & 0.8981374946 & 1.7015368483 \\ \mathrm{C} & -4.2228653897 & 2.7724169611 & 1.0764997836 \\ \mathrm{H} & -2.9884938706 & 3.2728953963 & -0.6121334401 \\ \mathrm{C} & -3.3961444893 & 1.0241021609 & 2.5132007212 \\ \mathrm{H} & -1.4795277926 & 0.2005575867 & 1.9753770831 \\ \mathrm{C} & -4.380271564 & 1.9584348631 & 2.2002194723 \\ \mathrm{H} & -4.9844204773 & 3.5054686726 & 0.8277557024 \\ \mathrm{H} & -3.4984332246 & 0.3965350366 & 3.3940367544 \\ \mathrm{H} & -5.2576346634 & 2.0616779686 & 2.8308341979\end{array}$


4,5-(BBN) 2-1,1-dimethyl-5-phenyl-2,5-dihydrosilole (7a) $\mathrm{E}=-1430.376943$

\begin{tabular}{|c|c|c|c|}
\hline $\mathrm{C}$ & -0.6547077716 & 0.5898018067 & -0.3760907852 \\
\hline $\mathrm{C}$ & 0.4967492877 & 0.0078187932 & -1.2107847415 \\
\hline $\mathrm{C}$ & 0.1998821358 & -0.2536481953 & -2.5073489219 \\
\hline $\mathrm{H}$ & -1.1141831204 & 0.4142707431 & -4.1066116784 \\
\hline $\mathrm{H}$ & -1.8577475156 & -0.7467468105 & -3.0037225827 \\
\hline $\mathrm{Si}$ & -1.6056194762 & 1.4731307431 & -1.8150121752 \\
\hline $\mathrm{C}$ & -1.1652761699 & 0.1057845338 & -3.0578490948 \\
\hline B & -1.5236109076 & -0.5923589541 & 0.2532660624 \\
\hline $\mathrm{C}$ & -1.030996769 & -2.0938511313 & 0.3351422487 \\
\hline $\mathrm{C}$ & -3.0213865025 & -0.504458449 & 0.7613694729 \\
\hline $\mathrm{H}$ & -0.0018404844 & -2.213375498 & -0.0134654196 \\
\hline $\mathrm{C}$ & -1.0704132686 & -2.50080593 & 1.8295483422 \\
\hline $\mathrm{C}$ & -1.9065890434 & -2.9850225901 & -0.5805034405 \\
\hline $\mathrm{C}$ & -3.1889307969 & -1.0833378411 & 2.1873666314 \\
\hline $\mathrm{H}$ & -3.429358889 & 0.5143727801 & 0.7682277867 \\
\hline $\mathrm{C}$ & -3.8126077258 & -1.3021696729 & -0.3195310853 \\
\hline $\mathrm{H}$ & -0.6817009345 & -3.5226194207 & 1.9422059408 \\
\hline $\mathrm{H}$ & -0.3763336116 & -1.8524639077 & 2.3849411508 \\
\hline $\mathrm{C}$ & -2.458912219 & -2.4085039301 & 2.4754460409 \\
\hline $\mathrm{C}$ & -3.4246659068 & -2.7797558329 & -0.4463152339 \\
\hline $\mathrm{H}$ & -1.6569884075 & -4.0425613374 & -0.4146402902 \\
\hline $\mathrm{H}$ & -1.6182727756 & -2.7655803477 & -1.6187932527 \\
\hline $\mathrm{H}$ & -2.8141445662 & -0.3341155307 & 2.8975244139 \\
\hline $\mathrm{H}$ & -4.2582380423 & -1.1982194182 & 2.4139393345 \\
\hline $\mathrm{H}$ & -3.6523604294 & -0.8319168934 & -1.300892097 \\
\hline $\mathrm{H}$ & -4.888776539 & -1.2136484971 & -0.1165381992 \\
\hline $\mathrm{H}$ & -3.0649573442 & -3.2585007652 & 2.1552765138 \\
\hline $\mathrm{H}$ & -2.3534497647 & -2.5195189066 & 3.5610753057 \\
\hline $\mathrm{H}$ & -3.9201326365 & -3.1983487256 & -1.3306277966 \\
\hline $\mathrm{H}$ & -3.8198812406 & -3.3398315714 & 0.4034333458 \\
\hline $\mathrm{H}$ & 0.939440551 & -0.7088081005 & -3.1652840695 \\
\hline B & 1.8780995954 & -0.4335599652 & -0.6149606701 \\
\hline $\mathrm{C}$ & 2.1699111175 & -0.7472653421 & 0.9071932765 \\
\hline $\mathrm{C}$ & 3.1865198144 & -0.618278149 & -1.4773710202 \\
\hline $\mathrm{H}$ & 1.285593855 & -0.6161220071 & 1.5456689943 \\
\hline $\mathrm{C}$ & 2.6123915041 & -2.2310485793 & 0.9982652294 \\
\hline $\mathrm{C}$ & 3.2621682815 & 0.2200529735 & 1.4393862881 \\
\hline $\mathrm{C}$ & 3.7293845671 & -2.0594004112 & -1.3146427391 \\
\hline $\mathrm{H}$ & 3.0283146167 & -0.4415620268 & -2.5491153036 \\
\hline $\mathrm{C}$ & 4.1507356066 & 0.491483417 & -0.9777748764 \\
\hline $\mathrm{H}$ & 2.8288659819 & -2.4801669987 & 2.046471933 \\
\hline $\mathrm{H}$ & 1.7749821833 & -2.8779001827 & 0.7031502611 \\
\hline $\mathrm{C}$ & 3.8306285045 & -2.5795336533 & 0.1305160963 \\
\hline $\mathrm{C}$ & 4.4834546405 & 0.4324374791 & 0.5217444103 \\
\hline $\mathrm{H}$ & 3.6066315674 & -0.1339517596 & 2.4215702068 \\
\hline $\mathrm{H}$ & 2.7862564252 & 1.1899299177 & 1.6169192161 \\
\hline $\mathrm{H}$ & 3.0531880616 & -2.7253977993 & -1.8699063834 \\
\hline $\mathrm{H}$ & 4.7104530145 & -2.146738565 & -1.8028429572 \\
\hline $\mathrm{H}$ & 3.6935850696 & 1.4667408186 & -1.203813135 \\
\hline
\end{tabular}




$\begin{array}{lccc}\mathrm{H} & 5.0832870268 & 0.4564236812 & -1.557634387 \\ \mathrm{H} & 4.7381525865 & -2.2016586259 & 0.6061548209 \\ \mathrm{H} & 3.9470323002 & -3.6696548872 & 0.1027245314 \\ \mathrm{H} & 4.9752644872 & 1.3703435137 & 0.8079004653 \\ \mathrm{H} & 5.2296300171 & -0.3469700932 & 0.6913230015 \\ \mathrm{C} & -0.7095975106 & 3.0406528331 & -2.3487058862 \\ \mathrm{H} & 0.3356365396 & 2.8467016205 & -2.604617501 \\ \mathrm{H} & -1.2084769169 & 3.444184711 & -3.2372093584 \\ \mathrm{H} & -0.7358563406 & 3.8061484591 & -1.5671507526 \\ \mathrm{C} & -3.3912451634 & 1.9957090335 & -1.5250476958 \\ \mathrm{H} & -4.1085526896 & 1.1866213352 & -1.3876826368 \\ \mathrm{H} & -3.4232477549 & 2.6346881529 & -0.6345527056 \\ \mathrm{H} & -3.7197544707 & 2.6055602871 & -2.3736009574 \\ \mathrm{C} & -0.2188244076 & 1.6545182873 & 0.6191765 \\ \mathrm{C} & 0.898890874 & 2.4570323691 & 0.3411904649 \\ \mathrm{C} & -0.914716799 & 1.9214678045 & 1.8082438112 \\ \mathrm{C} & 1.3053489469 & 3.4728950417 & 1.2019167721 \\ \mathrm{H} & 1.4576570758 & 2.2881520732 & -0.5763478826 \\ \mathrm{C} & -0.5084826631 & 2.9342123751 & 2.6764796198 \\ \mathrm{H} & -1.7870734714 & 1.3312384189 & 2.0670706117 \\ \mathrm{C} & 0.6048085043 & 3.7179165413 & 2.3807993182 \\ \mathrm{H} & 2.1769961855 & 4.0700774979 & 0.9487099658 \\ \mathrm{H} & -1.0665475671 & 3.1059330564 & 3.5923521752 \\ \mathrm{H} & 0.9215830117 & 4.5047904084 & 3.0577288158\end{array}$

3,4-(BBN) $)_{2}$-1,1,6,6-tetramethyl-5-trimethylsilyl-1,2,3,6-tetrahydro-1,6-disilapentalene (9b)

$\mathbf{E}=-2053.340804$

$\begin{array}{lclr}\mathrm{C} & 0.0139672565 & 1.001037669 & 0.5612073791 \\ \mathrm{C} & 0.8819943282 & -0.1888141609 & 0.1889746151 \\ \mathrm{C} & 2.0317824552 & 0.1244204379 & -0.4906788744 \\ \mathrm{C} & 0.5051287717 & 2.2457992828 & 0.3207756927 \\ \mathrm{C} & -2.0390366138 & 2.2415706593 & 1.3786742277 \\ \mathrm{C} & -1.3918356544 & 0.8267289459 & 1.0950294583 \\ \mathrm{C} & -0.1557046769 & 4.624401511 & 2.2504414971 \\ \mathrm{H} & 0.677314529 & 5.2604304736 & 1.9323196362 \\ \mathrm{H} & -0.9632641377 & 5.2791295143 & 2.5947033313 \\ \mathrm{H} & 0.1845047182 & 4.0233484889 & 3.0990911711 \\ \mathrm{C} & -1.3352152065 & 4.5939065412 & -0.5854907922 \\ \mathrm{H} & -2.2083776433 & 5.189464585 & -0.2980379847 \\ \mathrm{H} & -0.5506670342 & 5.2868173526 & -0.9082431281 \\ \mathrm{H} & -1.6088907903 & 3.9737887688 & -1.4457835877 \\ \mathrm{C} & 3.5866247912 & 2.6545853227 & 0.5157452328 \\ \mathrm{H} & 4.5550683939 & 2.4437890586 & 0.0520056824 \\ \mathrm{H} & 3.5081367683 & 3.7386813464 & 0.6509666644\end{array}$




\begin{tabular}{|c|c|c|c|}
\hline $\mathrm{H}$ & 3.5702924328 & 2.1882747888 & 1.5056729409 \\
\hline $\mathrm{C}$ & 2.1493031277 & 2.8251465804 & -2.2281611172 \\
\hline $\mathrm{H}$ & 1.4199680799 & 2.3369688424 & -2.8823789188 \\
\hline $\mathrm{H}$ & 1.8636381481 & 3.8788118581 & -2.1366291103 \\
\hline $\mathrm{H}$ & 3.1311061312 & 2.7811627726 & -2.7061244336 \\
\hline $\mathrm{Si}$ & -0.7300865559 & 3.5186328431 & 0.8387840446 \\
\hline $\mathrm{Si}$ & 2.1389066699 & 2.0275654225 & -0.5193099784 \\
\hline $\mathrm{Si}$ & 3.4818208946 & -0.8811170223 & -1.1531133805 \\
\hline $\mathrm{C}$ & 3.0851510141 & -2.3684142553 & -2.2545917208 \\
\hline $\mathrm{H}$ & 3.9502393314 & -2.5367060629 & -2.9062532321 \\
\hline $\mathrm{H}$ & 2.8864565977 & -3.2983614734 & -1.7199675975 \\
\hline $\mathrm{H}$ & 2.2255351134 & -2.1507139079 & -2.8971674421 \\
\hline $\mathrm{C}$ & 4.6347190529 & -1.3341942693 & 0.2743131358 \\
\hline $\mathrm{H}$ & 5.4843363425 & -1.9254205316 & -0.0850160833 \\
\hline $\mathrm{H}$ & 5.0286206695 & -0.4162832439 & 0.7251971458 \\
\hline $\mathrm{H}$ & 4.1332482591 & -1.900125429 & 1.0645655447 \\
\hline $\mathrm{C}$ & 4.4703780227 & 0.2528949364 & -2.3010903408 \\
\hline $\mathrm{H}$ & 3.8936617888 & 0.5129695694 & -3.1955236531 \\
\hline $\mathrm{H}$ & 4.7965842959 & 1.1821480765 & -1.8242003311 \\
\hline $\mathrm{H}$ & 5.3699365878 & -0.2787265289 & -2.6316346986 \\
\hline $\mathrm{H}$ & -2.9560052281 & 2.4205545583 & 0.7908327492 \\
\hline B & 0.6260988104 & -1.6056232195 & 0.8118887528 \\
\hline $\mathrm{C}$ & 0.326855229 & -1.7912718818 & 2.3548317924 \\
\hline $\mathrm{C}$ & 0.7244511055 & -3.0108258809 & 0.1002220572 \\
\hline $\mathrm{H}$ & 0.2362735546 & -0.8382838494 & 2.8914019682 \\
\hline $\mathrm{C}$ & -0.9966560999 & -2.5744952347 & 2.5491903461 \\
\hline $\mathrm{C}$ & 1.5748749241 & -2.510092384 & 2.927890894 \\
\hline $\mathrm{C}$ & -0.6788286607 & -3.6562004305 & 0.229620847 \\
\hline $\mathrm{H}$ & 0.9357092879 & -2.9280996084 & -0.9705654279 \\
\hline $\mathrm{C}$ & 1.8367375255 & -3.8696258147 & 0.7543625058 \\
\hline $\mathrm{H}$ & -1.1105498703 & -2.8494635761 & 3.6073312414 \\
\hline $\mathrm{H}$ & -1.8248010357 & -1.8867522213 & 2.3262965555 \\
\hline $\mathrm{C}$ & -1.17619485 & -3.8279207006 & 1.6714359511 \\
\hline $\mathrm{C}$ & 1.8683470537 & -3.8769757738 & 2.2950009495 \\
\hline $\mathrm{H}$ & 1.4626407794 & -2.6288362351 & 4.0140802918 \\
\hline $\mathrm{H}$ & 2.4437720144 & -1.8506603913 & 2.7846252087 \\
\hline $\mathrm{H}$ & -1.3928956316 & -3.038356777 & -0.3300120623 \\
\hline $\mathrm{H}$ & -0.6723087166 & -4.6337927231 & -0.2719169205 \\
\hline $\mathrm{H}$ & 2.8045998752 & -3.4943710562 & 0.4032553257 \\
\hline $\mathrm{H}$ & 1.7637925111 & -4.9025944019 & 0.3840447171 \\
\hline $\mathrm{H}$ & -2.2415379764 & -4.0906171452 & 1.650785536 \\
\hline $\mathrm{H}$ & 2.8599165235 & -4.2136892143 & 2.6215646109 \\
\hline $\mathrm{H}$ & 1.1666684349 & -4.6149581303 & 2.6897792676 \\
\hline B & -2.5178968171 & 0.425458098 & 0.0777146728 \\
\hline $\mathrm{C}$ & -2.4275442831 & 0.689913783 & -1.4701850694 \\
\hline $\mathrm{C}$ & -3.8797799855 & -0.2689680513 & 0.4733418271 \\
\hline $\mathrm{H}$ & -1.5286126418 & 1.2539289319 & -1.7572409949 \\
\hline $\mathrm{C}$ & -2.2567589078 & -0.7551663869 & -2.0207811172 \\
\hline $\mathrm{C}$ & -3.6653072324 & 1.4415373537 & -2.006132409 \\
\hline $\mathrm{C}$ & -3.8452475901 & -1.6765063425 & -0.1825322282 \\
\hline $\mathrm{H}$ & -3.9820647166 & -0.4141501381 & 1.5582557673 \\
\hline
\end{tabular}




$\begin{array}{llll}\mathrm{C} & -5.067021756 & 0.5911444631 & -0.0118483419 \\ \mathrm{H} & -2.1399188313 & -0.723550148 & -3.1125518449 \\ \mathrm{H} & -1.3073892881 & -1.1559310933 & -1.6301822012 \\ \mathrm{C} & -3.4025480885 & -1.7196091622 & -1.6614802789 \\ \mathrm{C} & -5.0315487912 & 0.9322773043 & -1.5096316592 \\ \mathrm{H} & -3.6593388332 & 1.4466892882 & -3.1054027331 \\ \mathrm{H} & -3.5645560501 & 2.4931357722 & -1.700064219 \\ \mathrm{H} & -3.1584872097 & -2.296967836 & 0.4065582537 \\ \mathrm{H} & -4.8325473343 & -2.1499262361 & -0.0863363398 \\ \mathrm{H} & -5.0791618974 & 1.5255740164 & 0.5672118226 \\ \mathrm{H} & -6.0137813157 & 0.082344832 & 0.217291082 \\ \mathrm{H} & -4.2552078405 & -1.5194902697 & -2.3137730471 \\ \mathrm{H} & -3.0888226337 & -2.7432060206 & -1.9027038648 \\ \mathrm{H} & -5.7867751311 & 1.6997487257 & -1.7169069647 \\ \mathrm{H} & -5.340725459 & 0.0584702628 & -2.0877414561 \\ \mathrm{H} & -0.6765658056 & -4.6872261548 & 2.1244193695 \\ \mathrm{H} & -2.344516398 & 2.3180085965 & 2.4260878649 \\ \mathrm{H} & -1.4086326615 & 0.2304836806 & 2.0114672676\end{array}$

\section{4,6a-(BBN) $)_{2}$-1,1,6,6-tetramethyl-5-trimethylsilyl-1,2,6,6a-tetrahydro-1,6-disilapentalene (10b)}

$\mathbf{E}=-2053.350704$

$\begin{array}{lccl}\mathrm{C} & 0.2232215483 & -0.577568664 & 0.9598795155 \\ \mathrm{C} & -0.9853269959 & 0.0872180443 & 0.4008293703 \\ \mathrm{C} & -0.8522867788 & 1.4236179334 & 0.1350750819 \\ \mathrm{C} & 1.5373152787 & 0.1972854783 & 0.8494159103 \\ \mathrm{C} & 1.5676205045 & -2.1719435525 & 2.2552633371 \\ \mathrm{C} & 0.2530591796 & -1.7494458678 & 1.630374146 \\ \mathrm{C} & 4.2542639121 & -0.3608291211 & 2.5882030926 \\ \mathrm{H} & 4.5789485118 & 0.6849347068 & 2.5412175797 \\ \mathrm{H} & 4.5357868379 & -0.7447267 & 3.5750906804 \\ \mathrm{H} & 4.8108588312 & -0.9202931914 & 1.8345746132 \\ \mathrm{C} & 1.6799468916 & 0.3011254509 & 4.003484607 \\ \mathrm{H} & 1.9880272703 & -0.317041023 & 4.8546680594 \\ \mathrm{H} & 2.045083298 & 1.316708133 & 4.1810873981 \\ \mathrm{H} & 0.5859267849 & 0.3233775634 & 3.9838758152 \\ \mathrm{C} & 1.9095805783 & 2.983224255 & -0.5623661032 \\ \mathrm{H} & 1.5936489165 & 4.0302466527 & -0.5738611495 \\ \mathrm{H} & 2.9612382971 & 2.9572185316 & -0.2514748901 \\ \mathrm{H} & 1.8546536379 & 2.5997319321 & -1.5859987275 \\ \mathrm{C} & 0.7774380577 & 3.008688615 & 2.233290383 \\ \mathrm{H} & 0.1909867702 & 2.509240363 & 3.0084237772 \\ \mathrm{H} & 1.7707351971 & 3.2337020476 & 2.6362097481 \\ \mathrm{H} & 0.2899013093 & 3.9626895597 & 2.0003109585 \\ \mathrm{Si} & 2.3789986162 & -0.4546542846 & 2.4222991122\end{array}$




\begin{tabular}{|c|c|c|c|}
\hline $\mathrm{Si}$ & 0.8818828443 & 1.9748308555 & 0.658055523 \\
\hline $\mathrm{Si}$ & -2.1414766606 & 2.6098657909 & -0.5425889587 \\
\hline $\mathrm{C}$ & -2.9280605638 & 1.9658022588 & -2.131144644 \\
\hline $\mathrm{H}$ & -3.5200217007 & 2.7589337941 & -2.6020106266 \\
\hline $\mathrm{H}$ & -2.1549865795 & 1.6520162108 & -2.8414742605 \\
\hline $\mathrm{H}$ & -3.5935001059 & 1.1161412938 & -1.9591993691 \\
\hline $\mathrm{C}$ & -1.2758383105 & 4.2268648023 & -0.9951607637 \\
\hline $\mathrm{H}$ & -2.0135146104 & 4.9424794534 & -1.3746570121 \\
\hline $\mathrm{H}$ & -0.7807379324 & 4.6914053273 & -0.1349644432 \\
\hline $\mathrm{H}$ & -0.525541554 & 4.0736091626 & -1.7776574316 \\
\hline $\mathrm{C}$ & -3.4748126716 & 3.035422894 & 0.7247749384 \\
\hline $\mathrm{H}$ & -4.1885591868 & 2.2203413088 & 0.8704577127 \\
\hline $\mathrm{H}$ & -3.0237101539 & 3.2658690734 & 1.6957559336 \\
\hline $\mathrm{H}$ & -4.0340792654 & 3.9174768314 & 0.3935959763 \\
\hline $\mathrm{H}$ & 2.1263853192 & -2.8506526038 & 1.592805488 \\
\hline B & -2.2466720176 & -0.8135918433 & 0.124611088 \\
\hline $\mathrm{C}$ & -3.6375570999 & -0.6672167311 & 0.8444596014 \\
\hline $\mathrm{C}$ & -2.231797522 & -2.0660365708 & -0.8389437688 \\
\hline $\mathrm{H}$ & -3.6966453629 & 0.2194907523 & 1.4852167539 \\
\hline $\mathrm{C}$ & -4.822882091 & -0.6432142628 & -0.1500490431 \\
\hline $\mathrm{C}$ & -3.6584110163 & -1.9100224099 & 1.7816296801 \\
\hline $\mathrm{C}$ & -3.2888440347 & -1.7701581974 & -1.9310602061 \\
\hline $\mathrm{C}$ & -2.5532133777 & -3.3623330265 & -0.0445127506 \\
\hline $\mathrm{H}$ & -5.7554675095 & -0.8693685549 & 0.38666472 \\
\hline $\mathrm{H}$ & -4.9326841981 & 0.3890354892 & -0.5039397996 \\
\hline $\mathrm{C}$ & -4.7112239838 & -1.5753351651 & -1.3789206377 \\
\hline $\mathrm{C}$ & -3.6568794914 & -3.2454204037 & 1.0265467062 \\
\hline $\mathrm{H}$ & -4.5460693946 & -1.8559229047 & 2.4254975373 \\
\hline $\mathrm{H}$ & -2.7931746979 & -1.8644557781 & 2.459182843 \\
\hline $\mathrm{H}$ & -2.9773074532 & -0.8814700764 & -2.4945933823 \\
\hline $\mathrm{H}$ & -3.3020735373 & -2.594847958 & -2.6565621875 \\
\hline $\mathrm{H}$ & -1.6278293444 & -3.6938701026 & 0.440472259 \\
\hline $\mathrm{H}$ & -2.8173905263 & -4.1593316144 & -0.7541606273 \\
\hline $\mathrm{H}$ & -5.1256387597 & -2.5584659551 & -1.1406065096 \\
\hline $\mathrm{H}$ & -5.3500684037 & -1.1782405223 & -2.177210442 \\
\hline $\mathrm{H}$ & -3.5368827406 & -4.0627383906 & 1.7478260852 \\
\hline $\mathrm{H}$ & -4.6426931905 & -3.3950857283 & 0.5784847893 \\
\hline $\mathrm{C}$ & 1.4625314432 & -0.6907075252 & -1.7883896193 \\
\hline B & 2.2291926593 & -0.0992219641 & -0.5350142393 \\
\hline $\mathrm{C}$ & 1.8176323713 & 0.0513505604 & -3.0938522206 \\
\hline $\mathrm{C}$ & 1.8652438642 & -2.1925328204 & -1.7890082594 \\
\hline $\mathrm{C}$ & 3.7844437415 & -0.0642675522 & -0.8137011052 \\
\hline $\mathrm{H}$ & 1.364114383 & -0.4694963897 & -3.9492032348 \\
\hline $\mathrm{H}$ & 1.3313749108 & 1.0364134791 & -3.0576536861 \\
\hline $\mathrm{C}$ & 3.3197167982 & 0.2441529315 & -3.3676185933 \\
\hline $\mathrm{C}$ & 3.3811417601 & -2.4515476019 & -1.8075450985 \\
\hline $\mathrm{H}$ & 1.3938993347 & -2.6968245011 & -2.6445847131 \\
\hline $\mathrm{H}$ & 1.4359639214 & -2.6545843589 & -0.8869374236 \\
\hline $\mathrm{C}$ & 4.1499545771 & 0.6468915088 & -2.1353063969 \\
\hline $\mathrm{H}$ & 4.3590970173 & 0.4135954281 & -0.0094271342 \\
\hline $\mathrm{C}$ & 4.1587495987 & -1.5787077751 & -0.8082452113 \\
\hline
\end{tabular}




$\begin{array}{cccr}\mathrm{H} & 3.4415910314 & 1.0142059631 & -4.1390021387 \\ \mathrm{H} & 3.7293555557 & -0.668005272 & -3.8071541758 \\ \mathrm{H} & 3.5645739613 & -3.5056542865 & -1.5662748464 \\ \mathrm{H} & 3.7753359859 & -2.3087514317 & -2.8153874854 \\ \mathrm{H} & 4.0380296461 & 1.7263854121 & -1.9762526544 \\ \mathrm{H} & 5.2149264537 & 0.4866155269 & -2.3553513977 \\ \mathrm{H} & 3.9726084346 & -1.9813915541 & 0.1973566951 \\ \mathrm{H} & 5.2385451951 & -1.6866188008 & -0.9824384187 \\ \mathrm{H} & -1.261506853 & -2.2134286482 & -1.3297411333 \\ \mathrm{H} & 0.3728771757 & -0.6545092907 & -1.6774091118 \\ \mathrm{H} & 1.4195353369 & -2.6962555303 & 3.2050856995 \\ \mathrm{H} & -0.6445628734 & -2.3478418687 & 1.7721958169\end{array}$

\section{Kinetic study of the equilibrium between $5 \mathrm{~d}$ and $7 \mathrm{~d}$.}

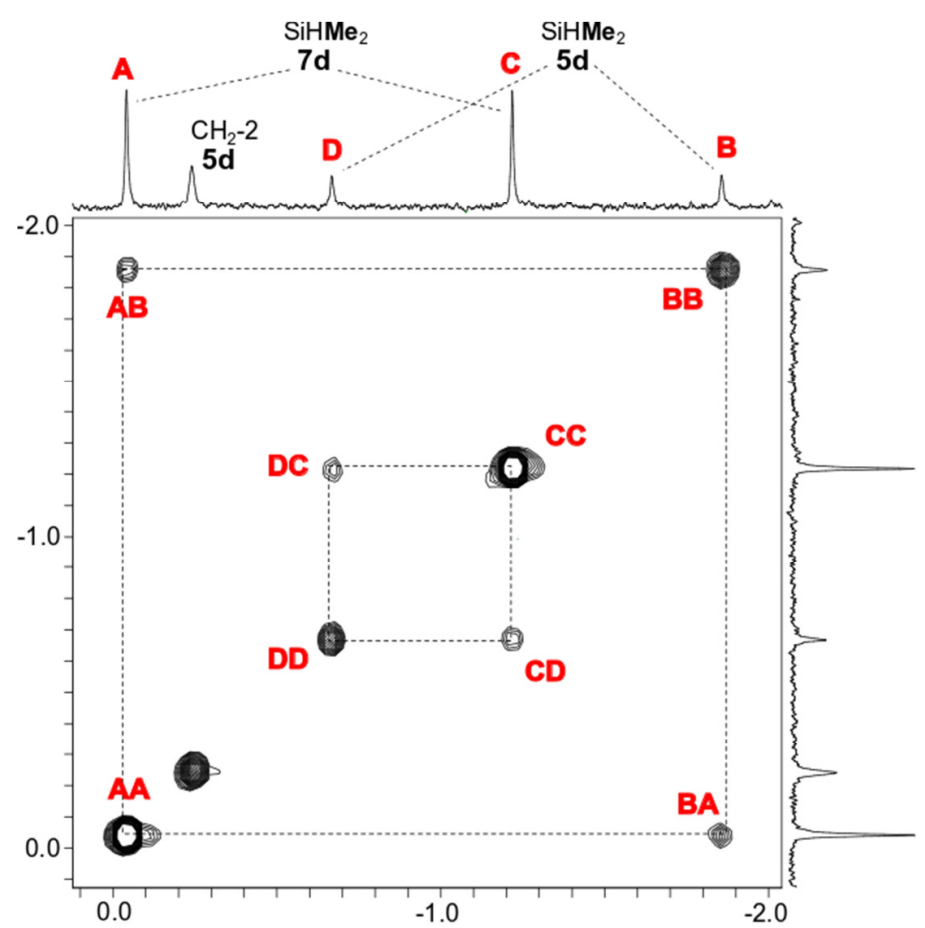

Figure S72. Integration of the diagonal and non-diagonal resonances in 2D EXSY spectra.
AA $\quad \mathbf{A B}$
CC CD
BA BB
DC DD 
The ratios of the isomers at each temperature were obtained from 2D EXSY spectra recorded with mixing of zero sec.

\section{$298 \mathrm{~K}$, isomers ratio: 4.49}

Mixing time $0.15 \mathrm{~s}$

10.0090 .709

$9.640 \quad 0.640$

$0.451 \quad 2.345$

$0.274 \quad 2.424$

Mixing time $0.20 \mathrm{~s}$

10.0970 .739

10.1540 .856

0.6112 .058

$0.667 \quad 2.405$

Mixing time $0.25 \mathrm{~s}$
$9.693 \quad 1.056$
$8.977 \quad 0.738$
$0.704 \quad 2.266$
$0.524 \quad 1.778$

$\underline{\text { Rate constants obtained as an average value from } 6 \text { measurements: }}$

$\mathrm{K}_{5 \mathrm{~d}-7 \mathrm{~d}}=1.154 \mathrm{~s}^{-1}$

$\mathrm{K}_{7 \mathrm{~d}-5 \mathrm{~d}}=0.438 \mathrm{~s}^{-1}$

\section{$303 \mathrm{~K}$, isomers ratio: 3.78}

Mixing time $0.15 \mathrm{~s}$
$9.843 \quad 0.864$
$9.226 \quad 0.642$
$0.729 \quad 2.234$
$0.640 \quad 2.372$

Mixing time $0.20 \mathrm{~s}$
$8.624 \quad 1.187$
$9.005 \quad 0.777$
$0.802 \quad 2.150$
$1.678 \quad 0.626$

Mixing time $0.25 \mathrm{~s}$
$8.455 \quad 1.147$
$8.767 \quad 1.109$
$1.037 \quad 1.914$
$0.851 \quad 1.657$ 
$\underline{\text { Rate constants obtained as an average value from } 6 \text { measurements: }}$

$\mathrm{K}_{5 \mathrm{~d}-7 \mathrm{~d}}=1.789 \mathrm{~s}^{-1}$

$\mathrm{K}_{7 \mathrm{~d}-5 \mathrm{~d}}=0.610 \mathrm{~s}^{-1}$

$308 \mathrm{~K}$, isomers ratio: 3.17

Mixing time $0.15 \mathrm{~s}$

$\begin{array}{llll}7.640 & 0.904 & 7.553 & 0.811 \\ 0.888 & 2.181 & 0.651 & 1.705\end{array}$

Mixing time $0.20 \mathrm{~s}$

$\begin{array}{llll}7.995 & 1.341 & 7.443 & 1.030 \\ 1.229 & 1.494 & 0.966 & 1.604\end{array}$

Mixing time $0.25 \mathrm{~s}$

$\begin{array}{llll}7.281 & 1.399 & 6.824 & 1.032\end{array}$

$\begin{array}{llll}1.201 & 1.655 & 0.856 & 1.383\end{array}$

$\underline{\text { Rate constants obtained as an average value from } 6 \text { measurements: }}$

$\mathrm{K}_{5 \mathrm{~d}-7 \mathrm{~d}}=2.561 \mathrm{~s}^{-1}$

$\mathrm{K}_{7 \mathrm{~d}-5 \mathrm{~d}}=0.894 \mathrm{~s}^{-1}$

$313 \mathrm{~K}$, isomers ratio: 2.82

Mixing time $0.10 \mathrm{~s}$

$\begin{array}{llll}7.702 & 0.878 & 7.425 & 0.749 \\ 0.821 & 2.492 & 0.669 & 1.790\end{array}$

Mixing time $0.15 \mathrm{~s}$

$\begin{array}{llll}8.274 & 1.216 & 7.485 & 1.208 \\ 1.109 & 2.133 & 0.978 & 1.568\end{array}$

Mixing time $0.20 \mathrm{~s}$ 
$7.205 \quad 1.438$

$7.082 \quad 1.584$

$1.372 \quad 1.588$

1.0801 .449

$\underline{\text { Rate constants obtained as an average value from } 6 \text { measurements: }}$

$\mathrm{K}_{5 \mathrm{~d}-7 \mathrm{~d}}=3.231 \mathrm{~s}^{-1}$

$\mathrm{K}_{7 \mathrm{~d}-5 \mathrm{~d}}=1.306 \mathrm{~s}^{-1}$

$318 \mathrm{~K}$, isomers ratio: 2.63

Mixing time $0.10 \mathrm{~s}$
$6.397 \quad 0.985$
$6.535 \quad 0.871$
$0.938 \quad 2.180$
1.1991 .708

Mixing time $0.15 \mathrm{~s}$
6.5651 .802
$6.156 \quad 1.508$
$1.714 \quad 2.180$
$1.199 \quad 1.708$

Mixing time $0.20 \mathrm{~s}$

$\begin{array}{llll}5.422 & 1.846 & 5.591 & 1.517 \\ 1.505 & 1.629 & 1.469 & 1.147\end{array}$

$\underline{\text { Rate constants obtained as an average value from } 6 \text { measurements: }}$

$\mathrm{K}_{5 \mathrm{~d}-7 \mathrm{~d}}=4.716 \mathrm{~s}^{-1}$

$\mathrm{K}_{7 \mathrm{~d}-5 \mathrm{~d}}=1.988 \mathrm{~s}^{-1}$ 


\section{FTIR spectra of $5 d / 7 d$ and $16 d$}

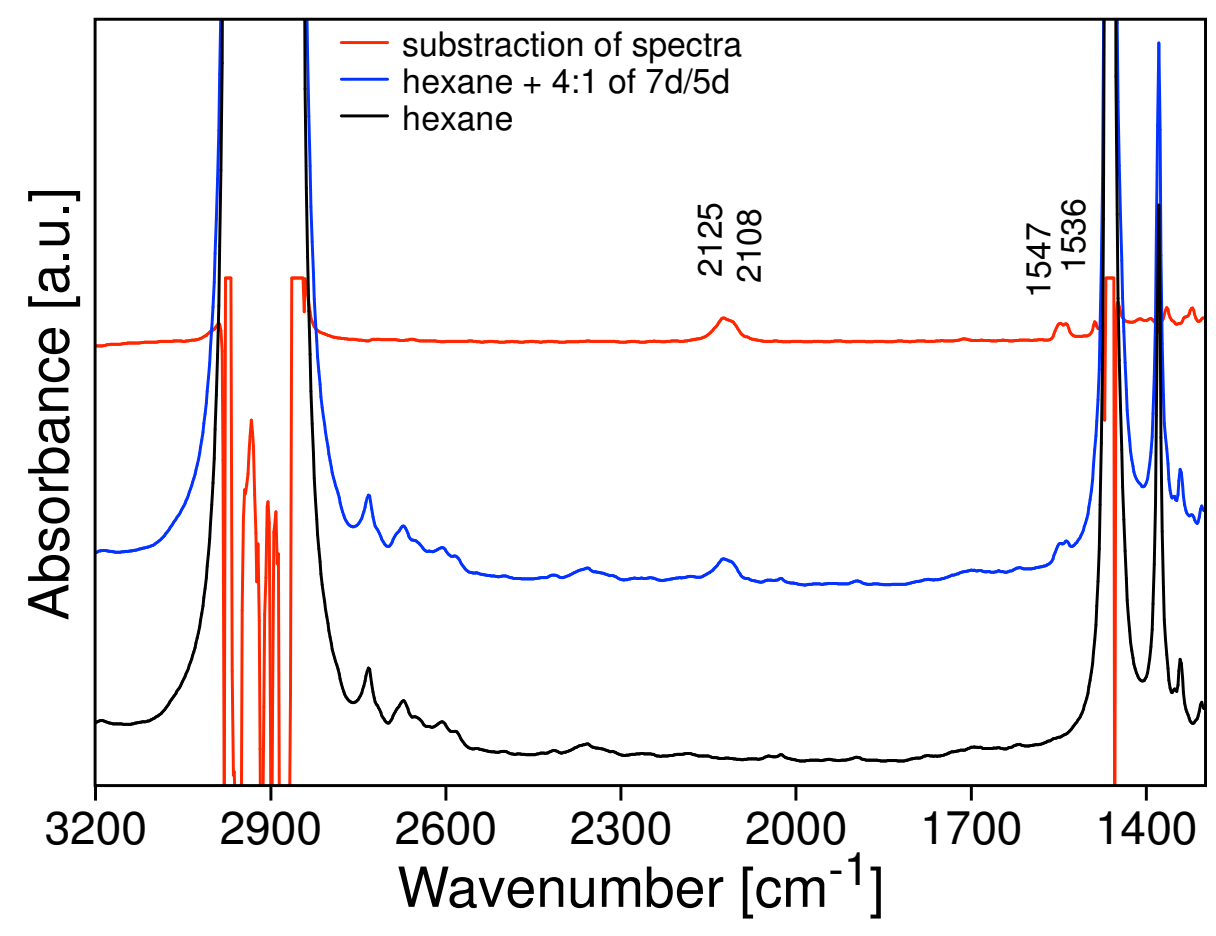

Figure S73. FTIR spectrum of the mixture $\mathbf{5 d} / 7 \mathbf{d}$ (hexane).

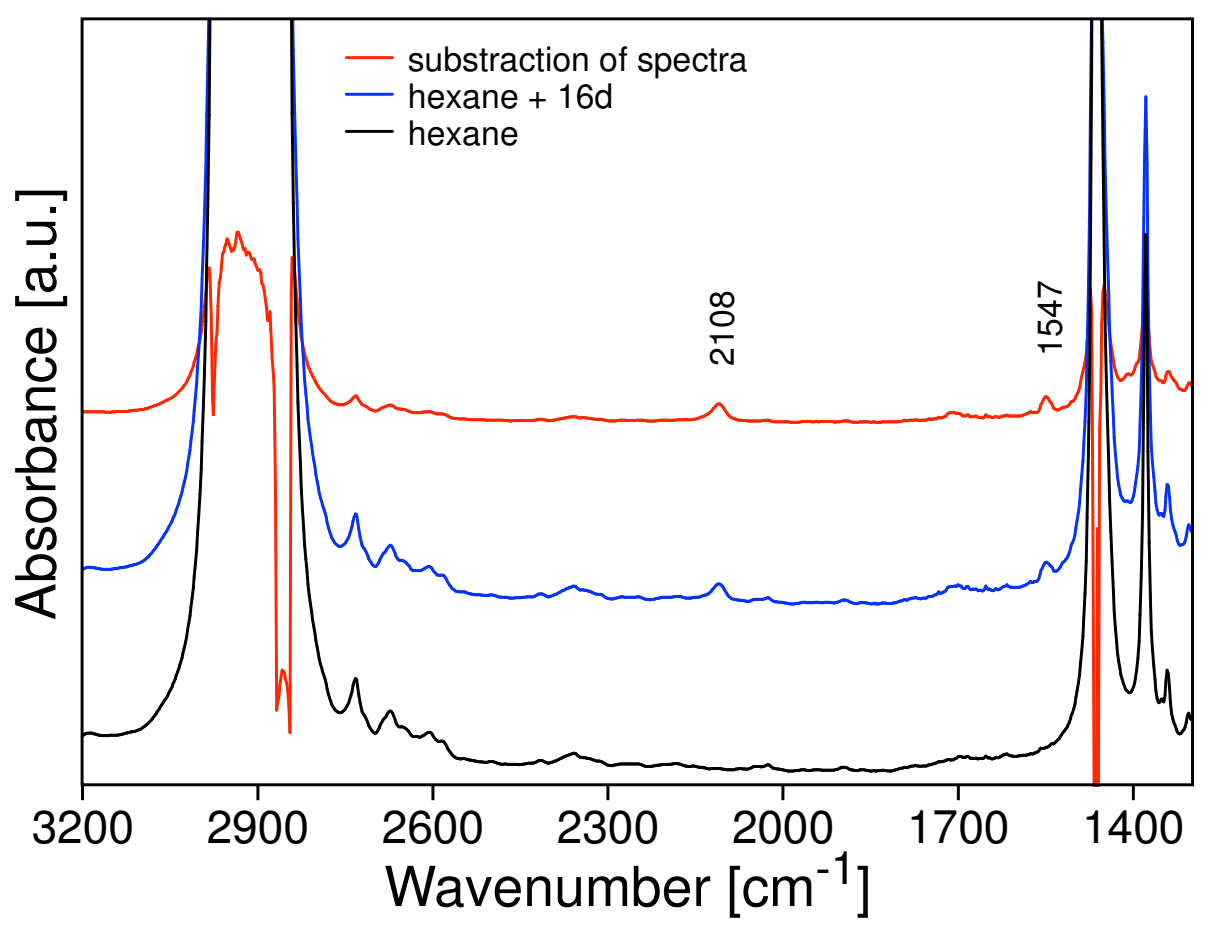

Figure S74. FTIR spectrum of 16d (hexane). 


\section{References}

[1] Petříček, V.; Dušek, M.; Palatinus, L. Crystallographic Computing System JANA2006: General features. Z. Anorg. Allg. Chem. 2014, 229 (5), 345.

[2] M. J. Frisch, G. W. Trucks, H. B. Schlegel, G. E. Scuseria, M. A. Robb, J. R. Cheeseman, G. Scalmani, V. Barone, B. Mennucci, G. A. Petersson, H. Nakatsuji, M. Caricato, X. Li, H. P. Hratchian, A. F. Izmaylov, J. Bloino, G. Zheng, J. L. Sonnenberg, M. Hada, M. Ehara, K. Toyota, R. Fukuda, J. Hasegawa, M. Ishida, T. Nakajima, Y. Honda, O. Kitao, H. Nakai, T. Vreven, J. A. Montgomery, J. E. Peralta, F. Ogliaro, M. Bearpark, J. J. Heyd, E. Brothers, K. N. Kudin, V. N. Staroverov, T. Keith, R. Kobayashi, J. Normand, K. Raghavachari, A. Rendell, J. C. Burant, S. S. Iyengar, J. Tomasi, M. Cossi, N. Rega, J. M. Millam, M. Klene, J. E. Knox, J. B. Cross, V. Bakken, C. Adamo, J. Jaramillo, R. Gomperts, R. E. Stratmann, O. Yazyev, A. J. Austin, R. Cammi, C. Pomelli, J. W. Ochterski, R. L. Martin, K. Morokuma, V. G. Zakrzewski, G. A. Voth, P. Salvador, J. J. Dannenberg, S. Dapprich, A. D. Daniels, O. Farkas, J. B. Foresman, J. V. Ortiz, J. Cioslowski and D. J. Fox, Gaussian 09, Revision D.01, Wallingford CT, 2013. 